\title{
The Coexistence of Blastocystis spp. in Humans, Animals and Environmental Sources from 2010-2021 in Asia
}

\author{
Adedolapo Aminat Rauff-Adedotun ${ }^{1}$ (D), Farah Haziqah Meor Termizi ${ }^{1}$, Nurshafarina Shaari ${ }^{2}$ and Ii Li Lee ${ }^{2, *}$ \\ 1 School of Biological Sciences, Universiti Sains Malaysia, Kuala Lumpur 11800, Malaysia; \\ adedotunameenah@gmail.com (A.A.R.-A.); farahhaziqah@usm.my (F.H.M.T.) \\ 2 Kulliyyah of Medicine \& Health Sciences, Universiti Islam Antarabangsa Sultan Abdul Halim Mu'adzam \\ Shah, Kuala Ketil 09300, Malaysia; shafarinashaari@unishams.edu.my \\ * Correspondence: leeiili@unishams.edu.my
}

check for

updates

Citation: Rauff-Adedotun, A.A.; Meor Termizi, F.H.; Shaari, N.; Lee, I.L. The Coexistence of

Blastocystis spp. in Humans, Animals and Environmental Sources from 2010-2021 in Asia. Biology 2021, 10, 990. https://doi.org/10.3390/ biology10100990

Academic Editors: Anastasios D. Tsaousis and Eleni Gentekaki

Received: 25 August 2021

Accepted: 24 September 2021

Published: 30 September 2021

Publisher's Note: MDPI stays neutral with regard to jurisdictional claims in published maps and institutional affiliations.

Copyright: (C) 2021 by the authors Licensee MDPI, Basel, Switzerland. This article is an open access article distributed under the terms and conditions of the Creative Commons Attribution (CC BY) license (https:// creativecommons.org/licenses/by/ $4.0 /)$.
Simple Summary: Blastocystis spp. are unicellular parasites that infect the gastrointestinal tract of humans and animals. Their occurrence in the environment had been detected in water sources, thus causing contamination. The presence of the parasites in humans, animals and environmental sources in Asia were reviewed according to countries in Asia, different categories of human and animal populations, and environmental sources including water samples, food and ambient air. The coexistence of the parasites poses a public health concern as the parasites are commonly found in most studies. Hence, there is a growing interest in the study of Blastocystis spp. Due to the isolation of Blastocystis spp. from living and non-living sources, a collaborative, multisectoral and transdisciplinary approach known as One Health is proposed for future study of Blastocystis spp. in order to achieve optimal health outcomes through the recognition of interconnection between people, animals and their shared environment.

Abstract: Blastocystis spp. are controversial unicellular protists that inhabit the gastrointestinal tract of humans and a wide range of animals worldwide. This review provides an overview of the prevalence and distribution of Blastocystis spp. and their subtypes throughout Asia. Research articles reporting on the presence of Blastocystis spp. in locations within Asia, between 1 January 2010, and 10 May 2021, were obtained from Scopus, PubMed, and Google Scholar. In 427 articles, the prevalence of Blastocystis spp. in 31 countries within the last decade was revealed. Isolates were found in humans, various mammals, birds, reptiles, insects, water sources, vegetables, and ambient air. Prevalence of Blastocystis spp. varied widely across host categories. Subtypes identified throughout Asia were STs 1-14, and ST18-22 (novel subtypes). ST1, ST2, ST3, ST4 were the most frequently isolated in humans; ST5 in pigs; ST10 and ST14 in goats, sheep, and cattle; and ST6 and ST7 in chickens. ST1 and ST3 were most common in water samples. ST1, ST2, ST3, ST4, ST5 and ST6 were shared by humans, animals, and water sources. There is a growing interest in the study of Blastocystis spp. and their subtypes in Asia. Due to the isolation of Blastocystis spp. from biotic and abiotic sources in Asia, the application of the One Health $(\mathrm{OH})$ approach to the study of Blastocystis spp. is proposed for improved perception of this organism.

Keywords: Blastocystis; subtypes; epidemiology; one health; Asia

\section{Introduction}

Blastocystis spp. are anaerobic unicellular eukaryotes that are widespread among humans and animals around the world [1-3]. They reside in the gastrointestinal tract wherein their role in gut health and disease is unresolved [4]. Several attempts were made at the classification of Blastocystis spp. by means of physiological and morphological characteristics [5], however, its place as a member of the phylum stramenopiles was revealed by Silberman et al. [6] based on phylogenetic analysis of the small subunit ribosomal rRNA (SSU rRNA) gene. 
Blastocystis spp. exhibit morphological and genetic polymorphism [7]. The six morphological forms described in the literature are vacuolar, granular, amoeboid, cyst, multivacuolar and avacuolar [8]; each of these forms show substantial variations in size [9]. Discerning one Blastocystis spp. isolate from another by morphological means alone poses a big challenge as isolates from different hosts appear similar [1].

Differences in the nucleotide sequences of the SSU rRNA gene of Blastocystis spp. isolates demonstrate the organism's substantial genetic heterogeneity $[10,11]$. According to a consensus on the terminology of Blastocystis spp. subtypes proposed by Stensvold et al. [10], Blastocystis spp. isolates are referred to as Blastocystis spp. 'subtypes' (STs). These designations are based on the differences among the SSU rRNA gene sequences; and by 2013, 17 different STs (ST1 to ST17) of Blastocystis spp. had been acknowledged [11]. Eleven additional STs (ST18 to ST28) have been proposed since then, although the validity of four of these STs (ST18 to ST20, and ST22) are being contested [12]. Infections with Blastocystis spp. ST1 to ST9 and ST12 have been reported in humans $[13,14]$. All Blastocystis spp. STs have, however, been widely isolated from non-human hosts, with the exception of ST9, whose first identification in a non-human host was by Noradilah et al. [15] in chickens reared by aborigines of rural Malaysian communities.

Blastocystis spp. are transmitted through the fecal-oral route via the ingestion of fecescontaminated food and water, with the cyst form as the only transmissible form $[9,14,16]$. Molecular epidemiological studies have revealed possible human-to-human, foodborne, waterborne and zoonotic transmission [17-27]. For example, Eroglu and Koltas [19] reported the isolation of Blastocystis spp. subtype 1 from Blastocystis spp. positive patients, their pets and the tap water they drank from. Likewise, the presence of Blastocystis spp. subtype 4 in humans, the animals they reared and the rivers they visited regularly were observed in a rural community in Nepal by Lee et al. [18]. It is also worth mentioning that Blastocystis spp. are included as waterborne pathogens in the World Health Organization's publications on drinking water quality [28], implying possible public health concerns.

Globally, increasing interactions between humans and animals (domestic, livestock, wildlife) at close proximity cannot be overemphasized. The rapid growth perceived in areas of agriculture, urbanization, industrialization, and international travel and trade have all contributed greatly to these interactions $[29,30]$. A human-animal-environment interface has emerged from the dynamic relationships between humans and animals; a clear understanding of the risks at this interface would allow better public health outcomes [29]. This is the One Health $(\mathrm{OH})$ holistic approach, which considers health in the context of human, animal and environmental relationships [31]. It urges the use of interdisciplinary collaborative effort to attain optimal health for humans, animals, plants, and the environment. Bearing in mind that the role of Blastocystis spp. in the host gut, whether as mutualists, commensals, or pathogens, has yet to be ascertained [32]; the study of this organism from an ecological standpoint is required.

Studies abound on the prevalence of Blastocystis spp. from around the world revealing the various host groups and geographic distribution of this intestinal protist. The growing use of polymerase chain reaction (PCR)-based approaches has, equally, broadened the understanding of genetic diversity and transmission of Blastocystis spp. Over the last decade, Blastocystis spp. research in Asia has noticeably intensified. Rauff-Adedotun et al. [33] observed an increase in the studies of Blastocystis spp. infection in animals in Southeast Asia over the last decade. This research direction is deemed timely considering the role of agriculture, industrialization and globalization on the rapid economic growth that is taking place in the Asian region; as well as the resulting large and growing human and livestock populations, high levels of interspecies interaction, and large-scale ecological change.

This article serves as a summary of the prevalence of Blastocystis spp. and the distribution of its subtypes in humans, animals, environmental, and food sources across Asia in the last decade. 


\section{Materials and Methods}

Articles on Blastocystis spp. research carried out within the continent of Asia were searched for in three electronic databases: Scopus, PubMed, and Google Scholar. The search covered articles published between 1 January 2010, and 10 May 2021. Duplicate articles from the three databases were removed; experiental studies, case reports, review articles, articles that did not report a positivity percentage and articles with unclear/confusing information were also excluded. Articles on the prevalence/occurrence and/or subtypes of Blastocystis spp. in both life and non-life sources undertaken within Asia were selected. The information extracted from each article included country of study, method(s) of detection of Blastocystis spp., host(s) of study, number of samples examined, number of samples positive, subtypes identified with corresponding numbers of isolates, author(s) and publication dates. Studies were retrieved on Blastocystis spp. in humans, various animal hosts, water sources, vegetables, and ambient air.

A total of 427 manuscripts met inclusion criteria, these studies were for 31 Asian countries/regions (Bangladesh, Cambodia, China, India, Indonesia, Iran, Iraq, Israel, Japan, Jordan, Korea, Laos, Lebanon, Malaysia, Myanmar, Nepal, Pakistan, Philippines, Qatar, Russia, Saudi Arabia, Singapore, Syria, Taiwan, Thailand, Turkey, Cyprus, United Arab Emirates, Uzbekistan, Vietnam, and Yemen). Blastocystis spp. have been identified in humans, different kinds of animals, leafy vegetables, water, and ambient air using conventional microscopy, in vitro cultivation, and molecular methods.

\section{Blastocystis spp. Infection in Humans}

Investigations on human Blastocystis spp. infections were on children, high school and college students, hospital patients/patients referred to medical laboratories for tests, patients with gastrointestinal disorder (GID) and other conditions, immunocompromised individuals, different categories of workers, and apparently healthy and general populations from urban and rural settings alike. Irrespective of these human host groups, Blastocystis spp. were the common organisms detected in studies describing gastrointestinal tract organisms in humans; and Blastocystis spp. ST1, ST2 and ST3 were the most frequently isolated.

The presence of Blastocystis spp. has been reported in infants, kindergarten, and schoolaged children in Asia in the past ten years (Table 1). However, the participants were either asymptomatic or their clinical conditions were not available. The majority of the studies were from Iran, Thailand, Malaysia, Turkey, and Indonesia; prevalence rates reported ranged from $1.2 \%$ to $83.7 \%$. Only about $24 \%$ of these studies reported on Blastocystis spp. subtypes. Subtypes identified were ST1, ST2, ST3, ST4, ST5, ST6 and ST7.

Table 1. Prevalence and subtype distribution of Blastocystis spp. in children in Asia (2010-2021).

\begin{tabular}{cccccc}
\hline Country & $\begin{array}{c}\text { No. of Samples } \\
\text { Examined }\end{array}$ & $\begin{array}{c}\text { No. of Positive } \\
\text { Samples (\%) }\end{array}$ & $\begin{array}{c}\text { Subtypes (STs) } \\
\text { Identified }\end{array}$ & Method(s) & References \\
\hline Bangladesh & 5679 & $795(14.0)$ & NA & CM, 1VC & Barua et al. [34] \\
Israel & 45,978 & $5422(11.8)$ & NA & CM, IVC & Ben-Shimol et al. [35] \\
China & 170 & $1(0.5)$ & NA & MOL & Zhang et al. [36] \\
China & 609 & $87(14.3)$ & ST1, ST2, ST3 & MOL & Qi et al. [37] \\
China & 466 & $71(15.2)$ & ST1, ST3, ST6, ST7 & IVC, MOL & Ning et al. [38] \\
Cambodia & 308 & $15(4.9)$ & NA & CM & Liao et al. [39] \\
India & 195 & $32(16.4)$ & NA & IVC, MOL & Yoshikawa et al. [40] \\
Indonesia & 492 & $147(29.9)$ & ST1, ST2, ST3 & MOL & Zulfa et al. [42] \\
Indonesia & 99 & $33(33.3)$ & ST1, ST2, ST3 & IVC, MOL & Sari et al. [43] \\
Indonesia & 141 & $58(41.1)$ & ST1, ST3, ST4 & CM & Subahar et al. [44] \\
Indonesia & 219 & $15(6.8)$ & NA & CM & Sari et al. [45] \\
Indonesia & 157 & $44(28.0)$ & NA & NA & Ashtiani et al. [46] \\
Iran & 124,366 & $3986(3.2)$ & ST1, ST2, ST3 & CM, IVC, MOL & Niaraki et al. [47] \\
Iran & 864 & $36(4.1)$ & NA & CM & Mahmoudvand et al. [48] \\
Iran & 366 & $37(3.1)$ & NA & CM & Abdi et al. [49] \\
Iran & 650 & $37(5.7)$ & &
\end{tabular}


Table 1. Cont.

\begin{tabular}{|c|c|c|c|c|c|}
\hline Country & $\begin{array}{l}\text { No. of Samples } \\
\text { Examined }\end{array}$ & $\begin{array}{l}\text { No. of Positive } \\
\text { Samples (\%) }\end{array}$ & $\begin{array}{l}\text { Subtypes (STs) } \\
\text { Identified }\end{array}$ & Method(s) & References \\
\hline Iran & 1100 & 149 (13.5) & NA & $\mathrm{CM}$ & Daryani et al. [50] \\
\hline Iran & 350 & $15(4.3)$ & NA & $\mathrm{CM}$ & Hazrati Tappeh et al. [51] \\
\hline Iran & 854 & $26(3.0)$ & NA & $\mathrm{CM}$ & Norouzi et al. [52] \\
\hline Iran & 200 & $35(17.5)$ & NA & $\mathrm{CM}$ & Babakhani et al. [53] \\
\hline Iran & 400 & $85(21.3)$ & NA & $\mathrm{CM}$ & Bahmani et al. [54] \\
\hline Iran & 306 & $9(2.9)$ & NA & $\mathrm{CM}$ & Saki and Amraee [55] \\
\hline Iran & 1465 & $31(2.1)$ & NA & $\mathrm{CM}$ & Turki et al. [56] \\
\hline Iraq & 107 & $4(3.7)$ & NA & $\mathrm{CM}$ & Mahdi and Al-Saadoon [57] \\
\hline Lebanon & 249 & $157(63.0)$ & ST1, ST2, ST3 & MOL & Osman et al. [58] \\
\hline Malaysia & 71 & $66(93.0)$ & NA & $\mathrm{CM}$ & Abd. Ghani and Yusof [59] \\
\hline Malaysia & 300 & $77(25.7)$ & NA & IVC & Abdulsalam et al. [60] \\
\hline Malaysia & 307 & $65(21.2)$ & NA & $\mathrm{CM}$ & Al-Harazi et al. [61] \\
\hline Malaysia & 342 & $4(1.2)$ & NA & $\mathrm{CM}$ & Sinniah et al. [62] \\
\hline Malaysia & 1760 & $186(10.6)$ & $\begin{array}{l}\text { ST1, ST2, ST3, } \\
\text { ST4, ST5 }\end{array}$ & IVC, MOL & Nithyamathi et al. [63] \\
\hline Malaysia & 116 & $2(1.7)$ & NA & $\mathrm{CM}$ & Tang and Kamel [64] \\
\hline Malaysia & 92 & $77(83.7)$ & NA & $\mathrm{CM}$ & Adli et al. [65] \\
\hline Nepal & 342 & $4(1.2)$ & NA & $\mathrm{CM}$ & Mukhiya et al. [66] \\
\hline Saudi Arabia & 1289 & $11(0.9)$ & NA & $\mathrm{CM}$ & Al-Mohammed et al. [67] \\
\hline Saudi Arabia & 581 & $10(1.7)$ & NA & $\mathrm{CM}$ & Bakarman et al. [68] \\
\hline Thailand & 203 & $9(4.4)$ & NA & $\mathrm{CM}$ & $\begin{array}{c}\text { Suntaravitun and } \\
\text { Dokmaikaw [69] }\end{array}$ \\
\hline Thailand & 1909 & $626(32.8)$ & NA & $\mathrm{CM}$ & Sanprasert et al. [70] \\
\hline Thailand & 370 & $118(31.9)$ & ST1, ST2, ST6 & MOL & Thathaisong et al. [71] \\
\hline Thailand & 233 & $29(12.5)$ & ST1, ST2, ST3 & IVC, MOL & Pipatsatitpong et al. [72] \\
\hline Thailand & 299 & $10(3.3)$ & NA & $\mathrm{CM}$ & Punsawad et al. [73] \\
\hline Thailand & 263 & $46(17.5)$ & NA & CM, IVC & Assavapongpaiboon et al. [74] \\
\hline Thailand & 331 & $44(13.3)$ & ST1, ST3 & IVC, MOL & Boondit et al. [75] \\
\hline Thailand & 202 & $3(1.5)$ & NA & $\mathrm{CM}$ & $\begin{array}{l}\text { Kitvatanachai and } \\
\text { Rhongbutsri [76] }\end{array}$ \\
\hline Thailand & 274 & $37(13.5)$ & NA & $\mathrm{CM}$ & Popruk et al. [77] \\
\hline Turkey & 195 & $28(14.4)$ & NA & $\mathrm{CM}$ & Güdücüoğlu et al. [78] \\
\hline Turkey & 328 & $77(23.5)$ & NA & $\mathrm{CM}$ & Hamamci et al. [79] \\
\hline Turkey & 468 & $35(7.4)$ & ST1, ST2, ST3, ST7 & CM, IVC, MOL & Sankur et al. [80] \\
\hline Turkey & 1181 & $7(0.6)$ & NA & $\mathrm{CM}$ & Calik et al. [81] \\
\hline Turkey & 219 & $97(44.3)$ & ST1, ST2, ST3, ST4 & MOL & Dogan et al. [82] \\
\hline
\end{tabular}

$\mathrm{CM}-$ Conventional microscopy, IVC-In vitro cultivation, MOL-Molecular technique, NA-Not applicable.

Prevalence and subtypes of Blastocystis spp. in immunocompromised individuals in Asia are summarized in Table 2. This category comprised mostly cancer, HIV / AIDS, and pulmonary tuberculosis patients. Reported prevalence rates were generally not above $30 \%$ except $54.8 \%$ in immunocompromised children with diarrhea in Indonesia, and $42.2 \%$ and $53.6 \%$ prevalence in HIV / AIDS cases and pulmonary tuberculosis patients respectively in Uzbekistan. Blastocystis spp. subtypes 1, 2, 3, 4, 5, and 7 were identified.

Table 2. Prevalence and subtype distribution of Blastocystis spp. in immunocompromised individuals in Asia (2010-2021).

\begin{tabular}{ccccccc}
\hline Host & Country & $\begin{array}{c}\text { No. of Samples } \\
\text { Examined }\end{array}$ & $\begin{array}{c}\text { No. of Positive } \\
\text { Samples (\%) }\end{array}$ & $\begin{array}{c}\text { Subtypes (STs) } \\
\text { Identified }\end{array}$ & Method(s) & References \\
\hline $\begin{array}{c}\text { Cancer patients } \\
\text { (children) }\end{array}$ & Iran & 52 & $11(21.2)$ & NA & CM & Salehi Kahish et al. [83] \\
$\begin{array}{c}\text { Cancer patients } \\
\text { (children) }\end{array}$ & Iran & 200 & $24(12.0)$ & ST1, ST2, ST3, & MOL & Asghari et al. [84] \\
$\begin{array}{c}\text { Cancer patients } \\
\text { (children) }\end{array}$ & Iran & 52 & $11(21.2)$ & NA & CM & Salehi Kahyesh et al. [85] \\
\hline
\end{tabular}


Table 2. Cont.

\begin{tabular}{|c|c|c|c|c|c|c|}
\hline Host & Country & $\begin{array}{l}\text { No. of Samples } \\
\text { Examined }\end{array}$ & $\begin{array}{l}\text { No. of Positive } \\
\text { Samples (\%) }\end{array}$ & $\begin{array}{l}\text { Subtypes (STs) } \\
\text { Identified }\end{array}$ & Method(s) & References \\
\hline $\begin{array}{l}\text { Cancer patients } \\
\text { (children) }\end{array}$ & Iran & 89 & $5(5.6)$ & NA & $\mathrm{CM}$ & Zabolinejad et al. [86] \\
\hline Cancer patients & Iran & 67 & $16(23.9)$ & NA & $\mathrm{CM}, \mathrm{MOL}$ & Mahmoudvand et al. [87] \\
\hline Cancer patients & China & 381 & $27(7.1)$ & ST1, ST3 & MOL & Zhang et al. [88] \\
\hline Cancer patients & Malaysia & 61 & $13(21.3)$ & NA & IVC & Chandramathi et al. [89] \\
\hline Cancer patients & $\begin{array}{l}\text { Saudi } \\
\text { Arabia }\end{array}$ & 138 & $38(27.5)$ & ST1, ST2, ST5 & MOL & Mohamed et al. [90] \\
\hline Cancer patients & Turkey & 232 & $25(10.8)$ & ST1, ST2, ST3 & CM, IVC, MOL & Yersal et al. [91] \\
\hline Cancer patients & Turkey & 201 & $29(14.4)$ & ST1, ST2, ST3 & $\mathrm{CM}, \mathrm{MOL}$ & Mulayim et al. [92] \\
\hline HIV / AIDS cases & China & 324 & $12(3.7)$ & $\begin{array}{l}\text { ST1, ST3, ST4, } \\
\text { ST7, ST12 }\end{array}$ & MOL & Teng et al. [93] \\
\hline HIV/AIDS cases & China & 720 & $154(21.4)$ & NA & IVC & Tian et al. [94] \\
\hline HIV / AIDS cases & China & 302 & $49(16.2)$ & NA & IVC & Tian et al. [95] \\
\hline HIV / AIDS cases & China & 79 & $11(13.9)$ & NA & IVC & Tian et al. [96] \\
\hline H IV/AIDS cases & China & 398 & $27(6.8)$ & NA & MOL & Zhang et al. [97] \\
\hline HIV / AIDS cases & China & 311 & $12(3.9)$ & $\begin{array}{l}\text { ST1, ST3, ST4, } \\
\text { ST7 }\end{array}$ & MOL & Zhang et al. [98] \\
\hline HIV / AIDS cases & China & 505 & $21(4.2)$ & NA & MOL & Zhu-Hua et al. [99] \\
\hline $\mathrm{HIV} /$ AIDS cases & India & 452 & $13(2.9)$ & NA & $\mathrm{CM}$ & Ramana et al. [100] \\
\hline HIV / AIDS cases & India & 200 & $14(7.0)$ & NA & $\mathrm{CM}$ & Khalil et al. [101] \\
\hline HIV / AIDS cases & Iran & 31 & $7(22.6)$ & NA & $\mathrm{CM}$ & Berenji et al. [102] \\
\hline $\mathrm{HIV} /$ AIDS cases & Iran & 60 & $10(16.7)$ & NA & $\mathrm{CM}$ & Yosefi et al. [103] \\
\hline HIV/AIDS cases & Iran & 356 & $14(3.9)$ & NA & $\mathrm{CM}$ & Agholi et al. [104] \\
\hline HIV/AIDS cases & Iran & 102 & $2(1.9)$ & NA & $\mathrm{CM}$ & Masoumi-Asl et al. [105] \\
\hline HIV / AIDS cases & Iran & 73 & $2(2.7)$ & NA & $\mathrm{CM}$ & Anvari-Tafti et al. [106] \\
\hline HIV / AIDS cases & Iran & 268 & $51(19.0)$ & $\begin{array}{l}\text { ST1, ST2, ST3, } \\
\text { ST4 }\end{array}$ & MOL & Piranshahi et al. [107] \\
\hline HIV / AIDS cases & Laos & 137 & $36(26.3)$ & NA & $\mathrm{CM}$ & Paboriboune et al. [108] \\
\hline $\mathrm{HIV} /$ AIDS cases & Nepal & 146 & $9(6.2)$ & NA & $\mathrm{CM}$ & Sherchan et al. 2012 [109] \\
\hline HIV/AIDS cases & Nepal & 112 & $1(0.9)$ & NA & $\mathrm{CM}$ & Ghimire et al. [110] \\
\hline HIV/AIDS cases & Turkey & 65 & $7(10.8)$ & NA & $\mathrm{CM}$ & Zorbozan et al. [111] \\
\hline HIV/AIDS cases & Uzbekistan & 500 & $211(42.2)$ & NA & & Davis et al. [112] \\
\hline Tuberculosis & Iran & 161 & $19(11.8)$ & ST1, ST2, ST3 & $\mathrm{CM}, \mathrm{MOL}$ & Taghipour et al. [113] \\
\hline Tuberculosis & Iran & 161 & $19(11.8)$ & NA & $\mathrm{CM}$ & Taghipour et al. [114] \\
\hline $\begin{array}{l}\text { Pulmonary } \\
\text { tuberculosis }\end{array}$ & Uzbekistan & 300 & $161(53.6)$ & NA & & Davis et al. [112] \\
\hline $\begin{array}{l}\text { Pulmonary } \\
\text { tuberculosis }\end{array}$ & China & 369 & $23(6.2)$ & NA & $\mathrm{CM}, 1 \mathrm{VC}$ & Li et al. [115] \\
\hline $\begin{array}{l}\text { Pulmonary } \\
\text { tuberculosis }\end{array}$ & China & 369 & $23(6.2)$ & NA & $\mathrm{CM}, 1 \mathrm{VC}$ & Li et al. [116] \\
\hline $\begin{array}{l}\text { pulmonary } \\
\text { tuberculosis }\end{array}$ & Iran & 50 & $9(18.0)$ & NA & $\mathrm{CM}$ & Taghipour et al. [117] \\
\hline $\begin{array}{l}\text { Renal transplant } \\
\text { recipients }\end{array}$ & Iran & 150 & $7(4.7)$ & NA & $\mathrm{CM}$ & Azami et al. [118] \\
\hline $\begin{array}{l}\text { Immunocompromised } \\
\text { children with } \\
\text { diarrhea }\end{array}$ & Indonesia & 42 & $23(54.8)$ & NA & IVC & Idris et al. [119] \\
\hline $\begin{array}{l}\text { Immunocompromised } \\
\text { children with } \\
\text { diarrhea }\end{array}$ & Turkey & 62 & $6(9.7)$ & NA & $\mathrm{CM}$ & Caner et al. [120] \\
\hline $\begin{array}{l}\text { Immunocompromised } \\
\text { patients }\end{array}$ & Iran & 265 & $11(4.2)$ & NA & $\mathrm{CM}$ & Rasti et al. [121] \\
\hline $\begin{array}{l}\text { Immunocompromised } \\
\text { patients }\end{array}$ & Iran & 204 & $62(30.4)$ & NA & $\mathrm{CM}$ & Izadi et al. [122] \\
\hline $\begin{array}{l}\text { Immunodeficient } \\
\text { patients }\end{array}$ & Iran & 190 & $32(16.8)$ & NA & $\mathrm{CM}$ & Esteghamati et al. [123] \\
\hline $\begin{array}{l}\text { Immunosuppressive } \\
\text { drugs recipient }\end{array}$ & Iran & 494 & $49(10.3)$ & NA & $\mathrm{CM}$ & Mirzaei et al. [124] \\
\hline $\begin{array}{l}\text { Immunocompromised } \\
\text { patients }\end{array}$ & $\begin{array}{l}\text { Saudi } \\
\text { Arabia }\end{array}$ & 136 & $7(5.2)$ & NA & $\mathrm{CM}$ & Al-Megrin et al. [125] \\
\hline $\begin{array}{l}\text { Common variable } \\
\text { immune deficiency } \\
\text { (CVID) syndrome } \\
\text { patients }\end{array}$ & Turkey & 37 & $3(8.1)$ & NA & $\mathrm{CM}$ & Uysal et al. [126] \\
\hline
\end{tabular}


It is noted that hematologic and non-hematologic (cranial) cancers with Blastocystis spp. infections are most commonly reported in children [82-85]. Whereas, colorectal, stomach, esophagus and non-gastrointestinal cancer such as lung, liver, breast, ovarian, hematologic and other cancers were detected in adults. Among the 10 studies focused on cancer patients, six studies clearly stated that cancer patients were receiving chemotherapy treatment $[82-85,87,88]$. One study recruited cancer patients who have not received any chemotherapy [89]. While the remaining two were classified as follow-up cases [90] and in- or out-patient cases [91], respectively. It is noted that the highest prevalence of Blastocystis spp. infection in cancer patients is detected in those who have not received chemotherapy [89] as compared to the other six studies. This could be due to the existing immunocompromised condition of the cancer patients that allowed an opportunistic infection to occur.

Patients with different gastrointestinal complaints and disorders such as constipation, abdominal pain, diarrhea, irritable bowel syndrome (IBS) and inflammatory bowel disease (IBD) have been examined for Blastocystis spp. infection with positive results recorded as shown in Table 3. The prevalence rate was as low as $0.5 \%$, with the highest being $67.1 \%$ and all isolates belonged to Blastocystis spp. subtypes 1, 2, 3, 4, 5, 6, and 7 .

Table 3. Prevalence and subtype distribution of Blastocystis spp. in humans with gastrointestinal symptoms in Asia (2010-2021).

\begin{tabular}{|c|c|c|c|c|c|c|}
\hline Host & Country & $\begin{array}{l}\text { No. of Samples } \\
\text { Examined }\end{array}$ & $\begin{array}{c}\text { No. of Positive } \\
\text { Samples (\%) }\end{array}$ & $\begin{array}{c}\text { Subtypes (STs) } \\
\text { Identified }\end{array}$ & Method(s) & References \\
\hline Children with diarrhea & China & 850 & $26(3.1)$ & NA & MOL & Zhang et al. [36] \\
\hline Children with diarrhea & Indonesia & 57 & $36(63.1)$ & $\begin{array}{c}\text { ST1, ST2, ST3, } \\
\text { ST4 }\end{array}$ & MOL & Zulfa et al. [42] \\
\hline Children with diarrhea & Turkey & 60 & $4(6.7)$ & NA & $\mathrm{CM}$ & Maçin et al. [127] \\
\hline Children with diarrhea & Iran & 400 & $8(2.0)$ & NA & $\mathrm{CM}$ & Asadi et al. [128] \\
\hline Children with diarrhea & Qatar & 580 & $27(4.7)$ & NA & MOL & Boughattas et al. [129] \\
\hline Children with diarrhea & Nepal & 588 & $5(0.9)$ & NA & $\mathrm{CM}$ & Dahal et al. [130] \\
\hline Children with diarrhea & Iran & 160 & $37(23.1)$ & NA & $\mathrm{CM}$ & Khalili et al. [131] \\
\hline Children with GID & Iran & 500 & $81(16.2)$ & NA & $\mathrm{CM}$ & Kiani et al. [132] \\
\hline Children with GID & Thailand & 82 & $13(15.9)$ & NA & CM, IVC & Awae et al. [133] \\
\hline Children with GID & Russia & 1273 & $62(4.9)$ & $\begin{array}{c}\text { ST1, ST2, ST3, } \\
\text { ST4, ST7 }\end{array}$ & $\mathrm{CM}, \mathrm{MOL}$ & Sigidaev et al. [134] \\
\hline Children with GID & Turkey & 84 & $18(21.4)$ & ST1, ST3, ST4 & MOL & Dogan et al. [82] \\
\hline Patients with diarrhea & Indonesia & 389 & $22(5.7)$ & NA & $\mathrm{CM}$ & Oyofo et al. [135] \\
\hline Patients with diarrhea & China & 271 & $13(4.8)$ & NA & MOL & Zhang et al. [136] \\
\hline Patients with diarrhea & Korea & 117 & $8(6.8)$ & NA & MOL & Won et al. [137] \\
\hline Patients with diarrhea & Iran & 134 & $28(20.9)$ & ST1, ST2, ST3 & CM, MOL & Jalallou et al. [138] \\
\hline Patients with diarrhea & Iran & 2023 & $1357(67.1)$ & NA & $\mathrm{CM}$ & Najafi et al. [139] \\
\hline Patients with GID & Iran & 1301 & $350(26.9)$ & NA & $\mathrm{CM}$ & Kiani et al. [140] \\
\hline Patients with GID & Iran & 287 & $65(22.7)$ & $\begin{array}{c}\text { ST1, ST2, ST3 } \\
\text { ST5 }\end{array}$ & IVC, MOL & Moosavi et al. [141] \\
\hline Patients with GID & Iran & $23^{*}$ & 23 & ST1 & CM, MOL & Shahbazi et al. [142] \\
\hline IBD patients & Iran & 71 & $9(12.7)$ & ST1, ST3 & IVC, MOL & Mirjalali et al. [143] \\
\hline Adolescents with IBS & Indonesia & 137 & $50(36.5)$ & $\mathrm{ST} 1, \mathrm{ST} 2, \mathrm{ST} 3$ & MOL & Kesuma et al. [144] \\
\hline IBS patients & India & 150 & $50(33.3)$ & ST1, ST3 & CM, IVC, MOL & Das et al. [145] \\
\hline IBS patients & Iran & 100 & $15(15.0)$ & NA & $\mathrm{CM}$ & Shafiei et al. [146] \\
\hline IBS patients & Iran & 122 & $24(19.7)$ & $\begin{array}{c}\text { ST1, ST3, ST4, } \\
\text { ST5 }\end{array}$ & MOL & Khademvatan et al. [147] \\
\hline IBS patients & Iraq & 78 & $38(48.7)$ & NA & CM, IVC & Sayal et al. [148] \\
\hline IBS patients & Thailand & 66 & $11(16.7)$ & NA & IVC & Surangsrirat et al. [149] \\
\hline Patients with GID & Iraq & 579 & $98(16.9)$ & NA & CM, IVC & Merza et al. [150] \\
\hline Patients with GID & Iraq & 249 & $92(36.9)$ & NA & $\mathrm{CM}$ & Mutlag et al. [151] \\
\hline Patients with GID & Thailand & 5 & $5(100.0)$ & ST3, ST6, ST7 & CM, IVC, MOL & Sanpool et al. [152] \\
\hline Patients with diarrhea & Turkey & 272 & $16(5.9)$ & NA & CM, MOL & Koltas et al. [153] \\
\hline Patients with GID & Turkey & 490 & $89(18.2)$ & NA & CM, IVC & Aykur et al. [154] \\
\hline Patients with GID & Turkey & 14,246 & $689(4.8)$ & NA & $\mathrm{CM}$ & Usluca et al. [155] \\
\hline Patients with GID & Turkey & 2334 & $134(5.7)$ & NA & $\mathrm{CM}$ & Cekin et al. [156] \\
\hline Patients with GID & Iran & 152 & $16(10.5)$ & ST1, ST2, ST3 & CM, IVC, MOL & Beiromvand et al. [157] \\
\hline Patients with diarrhea & Singapore & 193 & $1(0.5)$ & NA & CM, MOL & Feurle et al. [158] \\
\hline Patients with GID & $\begin{array}{l}\text { Saudi } \\
\text { Arabia }\end{array}$ & 114 & $15(13.2)$ & NA & $\mathrm{CM}$ & Hawash et al. [159] \\
\hline Patients with GID & Turkey & 5624 & $136(2.4)$ & NA & $\mathrm{CM}$ & Alver et al. [160] \\
\hline
\end{tabular}


Table 3. Cont.

\begin{tabular}{ccccccc}
\hline Host & Country & $\begin{array}{c}\text { No. of Samples } \\
\text { Examined }\end{array}$ & $\begin{array}{c}\text { No. of Positive } \\
\text { Samples (\%) }\end{array}$ & $\begin{array}{c}\text { Subtypes (STs) } \\
\text { Identified }\end{array}$ & Method(s) & References \\
\hline Patients with GID & Turkey & 17756 & $778(4.4)$ & NA & CM & Inceboz et al. [161] \\
Patients with GID & Iran & 670 & $38(5.7)$ & NA & IVC & Rostami Nejad et al. \\
Patients with GID & Pakistan & 339 & $59(17.4)$ & NA & CM & Haider et al. [163] \\
Patients with GID & Turkey & $29 *$ & 29 & ST1, ST2, ST3, & CM, MOL & Sakalar et al. [164] \\
\hline
\end{tabular}

* Study was carried out on Blastocystis sp. positive hosts, CM-Conventional microscopy, IVC—In vitro cultivation, MOL-Molecular technique, NA-Not applicable, GID—Gastrointestinal disorder, IBD-Inflammatory bowel disease, IBS-Irritable bowel syndrome.

The occurrence of Blastocystis spp. in mental rehabilitation centers was documented by several authors from Iran only (Table 4). Prevalence ranged from $4 \%$ to $55.2 \%$; and out of all nine of these studies, only one reported the use of molecular methods wherein ST1, ST3 and ST9 were identified.

Table 4. Prevalence and subtype distribution of Blastocystis spp. in mental rehabilitation centers in Asia (2010-2021).

\begin{tabular}{|c|c|c|c|c|c|c|}
\hline Host & Country & $\begin{array}{l}\text { No. of Samples } \\
\text { Examined }\end{array}$ & $\begin{array}{l}\text { No. of Positive } \\
\text { Samples (\%) }\end{array}$ & $\begin{array}{l}\text { Subtypes (STs) } \\
\text { Identified }\end{array}$ & Method(s) & References \\
\hline $\begin{array}{l}\text { Mentally disabled } \\
\text { children }\end{array}$ & Iran & 362 & $20(5.5)$ & NA & $\mathrm{CM}$ & Sharif et al. [165] \\
\hline $\begin{array}{l}\text { Mentally disabled } \\
\text { children and adults }\end{array}$ & Iran & 225 & $9(4.0)$ & NA & $\mathrm{CM}$ & Hazrati Tappeh et al. [166] \\
\hline Psychiatric patients & Iran & 65 & $15(23.1)$ & NA & $\mathrm{CM}$ & Khalili et al. [167] \\
\hline $\begin{array}{l}\text { Mentally disabled } \\
\text { individuals }\end{array}$ & Iran & 173 & $29(16.8)$ & NA & $\mathrm{CM}$ & Saeidinia et al. [168] \\
\hline $\begin{array}{l}\text { Mentally disabled } \\
\text { individuals }\end{array}$ & Iran & 133 & $12(9.0)$ & NA & $\mathrm{CM}$ & Shokri et al. [169] \\
\hline $\begin{array}{l}\text { Mentally disabled } \\
\text { individuals and } \\
\text { elderly people }\end{array}$ & Iran & 243 & $81(33.3)$ & NA & $\mathrm{CM}$ & Rasti et al. [170] \\
\hline $\begin{array}{l}\text { Mentally disabled } \\
\text { individuals }\end{array}$ & Iran & 126 & $38(30.2)$ & NA & $\mathrm{CM}$ & Mohammadi-Meskin et al. [171] \\
\hline $\begin{array}{l}\text { Mental retardation } \\
\text { center personnel }\end{array}$ & Iran & 37 & $12(32.4)$ & NA & $\mathrm{CM}$ & Mohammadi-Meskin et al. [171] \\
\hline $\begin{array}{l}\text { Schizophrenic male } \\
\text { patients }\end{array}$ & Iran & 58 & $32(55.2)$ & ST1, ST3, ST9 & $\mathrm{CM}, \mathrm{MOL}$ & Sheikh et al. [172] \\
\hline
\end{tabular}

$\mathrm{CM}$-Conventional microscopy, MOL—Molecular technique, NA-Not applicable.

Studies on the status of Blastocystis spp. infection in hospital in- and out-patients are shown in Table 5. The diseases/illnesses of these patients were, however, not stated in the reports. Nonetheless, they did not show any gastrointestinal-related symptoms and volunteered as healthy participants in the gastrointestinal studies. As a result of their involvement, though asymptomatic, they were detected positive for Blastocystis spp. infection. Infection rate as low as $0.02 \%$ was recorded in 23,278 Saudi Arabian patients, while all $(100 \%)$ of 15 hospital patients without any gastrointestinal complaints were found positive for Blastocystis spp. Asides Blastocystis spp. subtypes 1,2, and 3 which were the most commonly observed, STs 6 and 7 were also commonly identified while STs 4 and 5 were few. 
Table 5. Prevalence and subtype distribution of Blastocystis spp. in patients of health institutions in Asia (2010-2021) who volunteered in gastrointestinal studies.

\begin{tabular}{|c|c|c|c|c|c|}
\hline Country & $\begin{array}{l}\text { No. of Samples } \\
\text { Examined }\end{array}$ & $\begin{array}{l}\text { No. of Positive } \\
\text { Samples (\%) }\end{array}$ & $\begin{array}{l}\text { Subtypes (STs) } \\
\text { Identified }\end{array}$ & Method(s) & References \\
\hline China & 126 & $3(2.4)$ & ST5 & MOL & Zhu et al. [173] \\
\hline China & 198 & $21(10.6)$ & ST1, ST3, ST6, ST7 & MOL & Kang et al. [174] \\
\hline Iran & 670 & $23(3.4)$ & NA & IVC & Rostami Nejad et al. [162] \\
\hline Iran & 1232 & $154(12.6)$ & NA & $\mathrm{CM}$ & Abdipour et al. [175] \\
\hline Iran & 1383 & $239(17.3)$ & ST1, ST2, ST3 & CM, MOL & Bahrami et al. [176] \\
\hline Iran & 984 & $13(1.3)$ & NA & $\mathrm{CM}$ & Gholipoor et al. [177] \\
\hline Iran & 417 & $39(9.4)$ & NA & $\mathrm{CM}$ & Viesy et al. [178] \\
\hline Iran & 511 & $33(6.5)$ & ST2, ST3, ST5 & MOL & Badparva et al. [179] \\
\hline Iran & 420 & $60(14.3)$ & ST2, ST3 & $\mathrm{CM}, \mathrm{MOL}$ & Shaker et al. [180] \\
\hline Iran & 802 & $39(4.9)$ & ST1, ST2, ST3, ST7 & MOL & Haghighi et al. [181] \\
\hline Iran & 420 & $60(14.3)$ & NA & $\mathrm{CM}$ & Shaker et al. [182] \\
\hline Iran & 1120 & $65(5.8)$ & NA & $\mathrm{CM}$ & Tork et al. [183] \\
\hline Iran & 4788 & $247(5.2)$ & NA & $\mathrm{CM}$ & Asfaram et al. [184] \\
\hline Iran & 210 & $66(31.4)$ & $\begin{array}{l}\text { ST1, ST2, ST3, } \\
\text { ST4, ST5, ST6 }\end{array}$ & MOL & Bafghi et al. [185] \\
\hline Iran & 133 & $35(26.3)$ & ST1, ST2, ST3, ST5 & IVC, MOL & Moosavi et al. [141] \\
\hline Iran & 4427 & $407(9.2)$ & NA & & Karimazar et al. [186] \\
\hline Iraq & 300 & $146(48.7)$ & NA & $\mathrm{CM}$ & Abdul Ridha and Faieq, [187] \\
\hline Iran & 618 & $146(23.6)$ & ST1, ST2, ST3 & CM, IVC, MOL & Salehi et al. [188] \\
\hline Iran & 481 & $69(14.4)$ & $\begin{array}{c}\text { ST1, ST2, ST3, } \\
\text { ST4, ST5 }\end{array}$ & MOL & Khademvatan et al. [189] \\
\hline Iran & 250 & $41(16.4)$ & ST1, ST2, ST3 & CM, IVC, MOL & Sardarian et al. [190] \\
\hline Iran & 200 & $63(31.5)$ & NA & CM, IVC & Hamidi et al. [191] \\
\hline Iran & 5000 & $784(1.6)$ & NA & $\mathrm{CM}$ & Javadi et al. [192] \\
\hline Iran & 864 & $68(7.9)$ & ST1, ST2, ST3 & CM, IVC, MOL & Delshad et al. [193] \\
\hline Iran & 566 & $10(1.8)$ & NA & $\mathrm{CM}$ & Norouzi et al. [194] \\
\hline Iran & 100 & $13(13.0)$ & ST1, ST2, ST6 & CM, MOL & Sharifi et al. [195] \\
\hline Iran & 1878 & $152(8.1)$ & ST1, ST2, ST3, ST7 & $\mathrm{CM}, \mathrm{MOL}$ & Salehi et al. [196] \\
\hline Lebanon & 40 & $23(57.5)$ & ST1, ST2, ST3 & MOL & Greige et al. [197] \\
\hline Lebanon & 220 & $42(19.1)$ & ST1, ST2, ST3, ST4 & $\mathrm{CM}, \mathrm{MOL}$ & El Safadi et al. [198] \\
\hline Lebanon & 50 & $27(54.0)$ & ST1, ST2, ST3 & MOL & Greige et al. [199] \\
\hline Saudi Arabia & 23,278 & $5(0.02)$ & NA & $\mathrm{CM}$ & Imam et al. [200] \\
\hline Saudi Arabia & 130 & $3(2.3)$ & NA & $\mathrm{CM}$ & Hassen Amer et al. [201] \\
\hline Saudi Arabia & 1262 & $133(10.5)$ & ST1, ST2, ST3 & IVC, MOL & Mohamed et al. [202] \\
\hline Thailand & 14,325 & $199(1.4)$ & NA & $\mathrm{CM}$ & Laodim et al. [203] \\
\hline Thailand & 562 & $56(9.9)$ & ST1, ST3, ST6, ST7 & IVC, MOL & Jantermtor et al. [204] \\
\hline Thailand & 15 & $15(100.0)$ & ST1, ST3, ST6, ST7 & $\mathrm{CM}, \mathrm{IVC}, \mathrm{MOL}$ & Sanpool et al. [152] \\
\hline Turkey & 192 & $6(3.1)$ & NA & $\mathrm{CM}$ & Cekin et al. [156] \\
\hline Turkey & 20,948 & $13,245(63.2)$ & NA & $\mathrm{CM}$ & Polat et al. [205] \\
\hline Turkey & 50,185 & $275(0.5)$ & NA & $\mathrm{CM}$ & Beyhan et al. [206] \\
\hline Turkey & 4030 & $476(11.1)$ & ST1, ST2, ST3 & $\mathrm{CM}, \mathrm{MOL}$ & Sarzhanov et al. [207] \\
\hline Turkey & 6757 & $160(2.4)$ & NA & $\mathrm{CM}$ & Selek et al. [208] \\
\hline
\end{tabular}

CM-Conventional microscopy, IVC—In vitro cultivation, MOL-Molecular technique, NA—Not applicable.

Table 6 is a summary of Blastocystis spp. infection in students and working populations in Asia between 2010 and 2021. 
Table 6. Prevalence and subtype distribution of Blastocystis spp. in students and working populations in Asia (2010-2021).

\begin{tabular}{|c|c|c|c|c|c|c|}
\hline Host & $\begin{array}{l}\text { Country/ } \\
\text { Region }\end{array}$ & $\begin{array}{l}\text { No. of Samples } \\
\text { Examined }\end{array}$ & $\begin{array}{l}\text { No. of Positive } \\
\text { Samples (\%) }\end{array}$ & $\begin{array}{l}\text { Subtypes (STs) } \\
\text { Identified }\end{array}$ & Method(s) & References \\
\hline Adolescents & Indonesia & 70 & $20(28.6)$ & ST1, ST3 & MOL & Kesuma et al. [144] \\
\hline $\begin{array}{l}\text { High school } \\
\text { students foreign }\end{array}$ & Turkey & 192 & $63(32.8)$ & NA & & Yaman et al. [209] \\
\hline College students & China & $53^{*}$ & 53 & $\begin{array}{c}\text { ST1, ST3, ST4, } \\
\text { ST6, ST7 }\end{array}$ & IVC, MOL & Zhan et al. [210] \\
\hline $\begin{array}{l}\text { College students of } \\
\text { practical } \\
\text { parasitology } \\
\text { courses }\end{array}$ & Iran & 175 & $9(5.1)$ & NA & $\mathrm{CM}$ & Fallahi et al. [211] \\
\hline $\begin{array}{l}\text { Students who did } \\
\text { not take any } \\
\text { practical } \\
\text { parasitology } \\
\text { courses }\end{array}$ & Iran & 135 & $5(3.7)$ & NA & $\mathrm{CM}$ & Fallahi et al. [211] \\
\hline $\begin{array}{l}\text { University students } \\
\text { Working children }\end{array}$ & $\begin{array}{l}\text { Thailand } \\
\text { Iran }\end{array}$ & $\begin{array}{c}1025 \\
175\end{array}$ & $\begin{array}{l}416(40.6) \\
57(32.6)\end{array}$ & $\begin{array}{c}\text { ST1, ST2, ST3 } \\
\text { NA }\end{array}$ & $\begin{array}{c}\mathrm{CM}, \mathrm{IVC}, \mathrm{MOL} \\
\mathrm{CM}\end{array}$ & $\begin{array}{l}\text { Srichaipon et al. [212] } \\
\text { Salemi et al. [213] }\end{array}$ \\
\hline $\begin{array}{c}\text { Caregivers } \\
\text { in a childcare center }\end{array}$ & Thailand & 25 & $6(24.0)$ & $\mathrm{ST} 1, \mathrm{ST} 2, \mathrm{ST} 3$ & IVC, MOL & $\begin{array}{l}\text { Pipatsatitpong et al. } \\
\text { [72] }\end{array}$ \\
\hline Cattle breeders & Lebanon & 40 & $21(52.5)$ & ST1, ST2, ST3 & MOL & Greige et al. [197] \\
\hline $\begin{array}{l}\text { Chicken } \\
\text { slaughterhouse staff }\end{array}$ & Lebanon & 50 & $28(56.0)$ & $\begin{array}{c}\text { ST1, ST2, ST3, } \\
\text { ST6 }\end{array}$ & MOL & Greige et al. [199] \\
\hline $\begin{array}{l}\text { Pig handler and } \\
\text { individuals who } \\
\text { lived near pig farms }\end{array}$ & Thailand & 154 & $10(6.5)$ & ST1, ST3, ST5 & MOL & Pintong et al. [214] \\
\hline $\begin{array}{l}\text { Pig handler and } \\
\text { individuals who } \\
\text { lived near pig farms }\end{array}$ & Thailand & 117 & $15(12.8)$ & ST1, ST2, ST3 & MOL & Udonsom et al. [215] \\
\hline Food handlers & Iran & 210 & $3(1.4)$ & NA & $\mathrm{CM}$ & Kheirandish et al. [216] \\
\hline Food handlers & Iran & 1021 & $40(3.9)$ & NA & $\mathrm{CM}$ & Motazedian et al. [217] \\
\hline Food handlers & Iran & 1041 & $29(2.8)$ & NA & $\mathrm{CM}$ & Sharif et al. [218] \\
\hline Food handlers & Iran & 800 & $194(24.3)$ & NA & $\mathrm{CM}$ & $\begin{array}{l}\text { Heydari-Hengami et al } \\
{[219]}\end{array}$ \\
\hline Food handlers & Iran & 1018 & $7(7.2)$ & NA & $\mathrm{CM}$ & $\begin{array}{c}\text { Khodabakhsh } \\
\text { Arbat et al. [220] }\end{array}$ \\
\hline $\begin{array}{l}\text { Food handlers } \\
\text { food handlers }\end{array}$ & $\begin{array}{l}\text { Iran } \\
\text { Iran }\end{array}$ & $\begin{array}{c}1530 \\
816\end{array}$ & $\begin{array}{l}44(2.9) \\
18(2.2)\end{array}$ & $\begin{array}{l}\text { NA } \\
\text { NA }\end{array}$ & $\begin{array}{l}\mathrm{CM} \\
\mathrm{CM}\end{array}$ & $\begin{array}{l}\text { Shahnazi et al. [221] } \\
\text { Kheirandish et al. [222] }\end{array}$ \\
\hline Food handlers & Jordan & 901 & $6(0.7)$ & NA & $\mathrm{CM}$ & $\begin{array}{c}\text { Abdel-Dayem et al. } \\
\text { [223] }\end{array}$ \\
\hline Military personnel & Iraq & 437 & $36(8.2)$ & NA & $\mathrm{CM}$ & Downs et al. [224] \\
\hline Gardeners & Thailand & 253 & $23(9.1)$ & NA & $\mathrm{CM}$ & $\begin{array}{l}\text { Kitvatanachai and } \\
\text { Rhongbutsri, [225] }\end{array}$ \\
\hline Immigrant workers & Thailand & 600 & $6(1.0)$ & NA & $\mathrm{CM}$ & Sangwalee et al. [226] \\
\hline Immigrant workers & Qatar & 608 & $432(71.1)$ & $\mathrm{ST} 1, \mathrm{ST} 2, \mathrm{ST} 3$ & MOL & Abu-Madi et al. [227] \\
\hline Immigrant workers & Qatar & 735 & $479(65.2)$ & NA & $\mathrm{CM}, \mathrm{MOL}$ & Abu-Madi et al. [228] \\
\hline Settled immigrant & Qatar & 9208 & $398(4.3)$ & NA & $\mathrm{CM}$ & Abu-Madi et al. [229] \\
\hline $\begin{array}{l}\text { Newly arrived } \\
\text { immigrants }\end{array}$ & Qatar & 2486 & $137(5.5)$ & NA & MOL & Abu-Madi et al. [230] \\
\hline Settled immigrants & Qatar & 29,286 & $1010(3.5)$ & NA & MOL & Abu-Madi et al. [231] \\
\hline Resident workers & Qatar & 772 & $39(5.1)$ & NA & $\mathrm{CM}$ & Abu-Madi et al. [232] \\
\hline Workers & Saudi Arabia & 1238 & $245(19.8)$ & NA & $\mathrm{CM}$ & Wakid [233] \\
\hline $\begin{array}{l}\text { New employees } \\
\text { in a tertiary health } \\
\text { care center }\end{array}$ & Saudi Arabia & 2490 & $314(12.6)$ & NA & & Ahmed et al. [234] \\
\hline Foreign laborers & Taiwan & 7360 & $190(2.6)$ & NA & $\mathrm{CM}$ & Hsieh et al. [235] \\
\hline Foreigners & Taiwan & 2875 & $33(1.1)$ & NA & $\mathrm{CM}$ & Hsieh et al. [236] \\
\hline $\begin{array}{c}\text { Indonesian } \\
\text { immigrant workers } \\
\text { Sanitary and }\end{array}$ & Taiwan & 128 & $28(21.9)$ & ST1, ST2, ST3 & $\mathrm{CM}, \mathrm{MOL}$ & Chen et al. [237] \\
\hline $\begin{array}{c}\text { Non-sanitary } \\
\text { Institutions' } \\
\text { workers }\end{array}$ & Turkey & 2443 & $175(7.2)$ & NA & $\mathrm{CM}$ & Karaman et al. [238] \\
\hline Migrant workers & Malaysia & 220 & $68(30.9)$ & $\mathrm{ST} 1, \mathrm{ST} 2, \mathrm{ST} 3$ & IVC, MOL & Sahimin et al. [239] \\
\hline
\end{tabular}

* Study was carried out on Blastocystis spp. positive hosts, CM-Conventional microscopy, IVC—In vitro cultivation, MOL-Molecular technique, NA-Not applicable. 
Food handlers and immigrant workers were commonly screened in Iran and Qatar, respectively. In addition to Blastocystis spp. subtypes 1, 2, and 3; ST6 was isolated from chicken slaughterhouse staff in Lebanon [199], and ST5 in pig handlers in Thailand [214].

The majority of the studies on Blastocystis spp. infections in humans in Asia within 2010 and 2021 were on general populations of apparently healthy status; such participants comprised urban dwellers, rural dwellers, and healthy control for immunocompromised persons. As depicted in Table 7, low prevalence rates of less than $5 \%$ and rates as high as $50 \%$ were reported from the different countries where these studies were undertaken, and various techniques were used for the detection of this protist. Blastocystis spp. subtypes reported were STs1-7 and ST10, whose only record was from Lebanon.

Table 7. Prevalence and subtype distribution of Blastocystis spp. in apparently healthy general populations in Asia (2010-2021).

\begin{tabular}{|c|c|c|c|c|c|}
\hline Country & $\begin{array}{c}\text { No. of Samples } \\
\text { Examined }\end{array}$ & $\begin{array}{l}\text { No. of Positive } \\
\text { Samples (\%) }\end{array}$ & $\begin{array}{l}\text { Subtypes (STs) } \\
\text { Identified }\end{array}$ & Method(s) & References \\
\hline Bangladesh & 140 & $51(36.4)$ & NA & $\mathrm{CM}$ & Noor et al. [240] \\
\hline Cambodia & 218 & $40(18.4)$ & NA & $\mathrm{CM}$ & Schär et al. [241] \\
\hline Cambodia & 210 & $116(55.2)$ & ST1, ST2, ST3 & MOL & Wang et al. [242] \\
\hline China & 497 & $215(43.3)$ & NA & $\mathrm{CM}$ & He et al. [243] \\
\hline China & 5939 & $494(8.3)$ & NA & MOL & Chen et al. [244] \\
\hline China & 26,886 & $2(0.01)$ & NA & $\mathrm{CM}$ & Umar et al. [245] \\
\hline China & 1023 & $1(0.1)$ & NA & $\mathrm{CM}$ & Jiang [246] \\
\hline China & 6710 & $19(0.3)$ & NA & $\mathrm{CM}$ & Zhang et al. [247] \\
\hline China & 303 & $67(22.1)$ & NA & IVC & Tian et al. [95] \\
\hline China & 294 & $64(21.8)$ & NA & IVC & Tian et al. [96] \\
\hline China & 149 & $9(6.0)$ & NA & MOL & Zhang et al. [136] \\
\hline China & 366 & $28(7.6)$ & NA & $\mathrm{CM}, 1 \mathrm{VC}$ & Li et al. [115] \\
\hline China & 289 & $13(4.5)$ & ST1, ST3, ST4 & MOL & Gong et al. [248] \\
\hline China & 507 & $48(9.5)$ & ST1, ST2, ST3, ST4 & MOL & Deng et al. [249] \\
\hline China & 1118 & $390(34.9)$ & ST2, ST5 & MOL & Ma et al. [250] \\
\hline Cyprus & 230 & $64(27.8)$ & $\begin{array}{l}\text { ST1, ST2, ST3, ST4, } \\
\text { ST6, ST7 }\end{array}$ & MOL & Seyer et al. [251] \\
\hline India & 279 & 105 (37.6) & NA & MOL & Padukone et al. [252] \\
\hline India & 200 & $16(8.0)$ & NA & $\mathrm{CM}$ & Khalil et al. [101] \\
\hline India & 100 & $15(15.0)$ & ST1, ST3 & CM, IVC, MOL & Das et al. [145] \\
\hline India & 23 & $13(56.5)$ & NA & MOL & Lappan et al. [253] \\
\hline Indonesia & 646 & $15(2.3)$ & NA & $\mathrm{CM}$ & Wiria et al. [254] \\
\hline Indonesia & 54 & $5(9.3)$ & NA & IVC & Yulfi et al. [255] \\
\hline Indonesia & 424 & $146(34.4)$ & NA & $\mathrm{CM}$ & Sungkar et al. [256] \\
\hline Indonesia & 53 & $9(17.0)$ & NA & $\mathrm{CM}$ & Hayashi et al. [257] \\
\hline Iran & 5073 & $368(7.3)$ & NA & $\mathrm{CM}$ & Turgay et al. [258] \\
\hline Iran & 399 & $16(4.0)$ & NA & $\mathrm{CM}$ & Mahmoudi et al. [259] \\
\hline Iran & 130 & $40(30.1)$ & ST1, ST2, ST3 & CM, IVC, MOL & Beiromvand et al. [157] \\
\hline Iran & 20 & $3(15.0)$ & NA & $\mathrm{CM}$ & Berenji et al. [102] \\
\hline Iran & 166 & $35(21.1)$ & ST1, ST2, ST3 & IVC, MOL & Mirjalali et al. [143] \\
\hline Iran & 181 & $17(9.4)$ & NA & $\mathrm{CM}$ & Taghipour et al. [114] \\
\hline Iran & 225 & $5(2.2)$ & NA & $\mathrm{CM}$ & Azami et al. [118] \\
\hline Iran & 166 & $35(21.1)$ & ST1, ST2, ST3 & $\mathrm{CM}, \mathrm{MOL}$ & Jalallou et al. [138] \\
\hline Iran & 147 & $0(0.0)$ & NA & $\mathrm{CM}$ & Anvari-Tafti et al. [106] \\
\hline Iran & 122 & $21(17.2)$ & ST1, ST3, ST4, ST5 & MOL & Khademvatan et al. [147] \\
\hline Iran & 100 & $6(6.0)$ & NA & $\mathrm{CM}$ & Shafiei et al. [146] \\
\hline Iran & 67 & $6(9.0)$ & NA & $\mathrm{CM}, \mathrm{MOL}$ & Mahmoudvand et al. [87] \\
\hline Iran & 250 & $41(16.4)$ & ST1, ST2, ST3 & CM, IVC, MOL & Sardarian et al. [190] \\
\hline Iran & 1410 & $47(3.3)$ & ST3, ST4, ST5, ST7 & $\mathrm{CM}, \mathrm{MOL}$ & Khoshnood et al. [260] \\
\hline Iran & 655 & $180(27.5)$ & NA & $\mathrm{CM}$ & Pestehchian et al. [261] \\
\hline Iran & 5743 & $54(0.9)$ & NA & $\mathrm{CM}$ & Sadeghi et al. [262] \\
\hline Iran & 5739 & $30(0.5)$ & NA & $\mathrm{CM}$ & Sadeghi and Borji [263] \\
\hline Iran & 2838 & $139(5.0)$ & NA & $\mathrm{CM}$ & Badparva et al. 2014 [264] \\
\hline Iran & 1060 & 145 (13.7) & NA & $\mathrm{CM}$ & Mahni et al. [265] \\
\hline
\end{tabular}


Table 7. Cont.

\begin{tabular}{|c|c|c|c|c|c|}
\hline Country & $\begin{array}{c}\text { No. of Samples } \\
\text { Examined }\end{array}$ & $\begin{array}{c}\text { No. of Positive } \\
\text { Samples (\%) }\end{array}$ & $\begin{array}{c}\text { Subtypes (STs) } \\
\text { Identified }\end{array}$ & Method(s) & References \\
\hline Iran & 880 & $55(6.3)$ & NA & $\mathrm{CM}$ & Tork et al. [266] \\
\hline Iran & 652 & $48(7.4)$ & NA & $\mathrm{CM}$ & Jafari et al. [267] \\
\hline Iran & 561 & $159(28.4)$ & NA & $\mathrm{CM}$ & Hemmati et al. [268] \\
\hline Iran & 554 & $93(16.8)$ & NA & CM, IVC & Riabi et al. [269] \\
\hline Iran & 345 & $85(24.6)$ & ST1, ST2, ST3 & CM, IVC, MOL & Mardani Kataki et al. [270] \\
\hline Iran & 861 & $114(13.2)$ & NA & $\mathrm{CM}$ & $\begin{array}{c}\text { Abbaszadeh Afshar et al. } \\
\text { [271] }\end{array}$ \\
\hline Iran & 732 & $63(6.3)$ & NA & $\mathrm{CM}$ & Sobati [272] \\
\hline Iran & 184 & $45(24.5)$ & ST1, ST2, ST3 & MOL & Shirvani et al.[273] \\
\hline Iran & 283 & $20(7.1)$ & NA & $\mathrm{CM}$ & Barati et al.[274] \\
\hline Iran & 2838 & $129(4.5)$ & NA & $\mathrm{CM}$ & Badparva et al. [264] \\
\hline Iran & 565 & $144(25.5)$ & NA & $\mathrm{CM}$ & $\begin{array}{l}\text { Bairami Kuzehkanani et al. } \\
\text { [275] }\end{array}$ \\
\hline Iran & 1025 & $182(17.8)$ & NA & $\mathrm{CM}$ & Sarkari et al. [276] \\
\hline Iran & 1500 & $13(0.9)$ & NA & $\mathrm{CM}$ & Sharifdini et al. [277] \\
\hline Iran & 4788 & $277(5.8)$ & NA & $\mathrm{CM}$ & Pagheh et al. [278] \\
\hline Iran & 1008 & $46(4.6)$ & NA & $\mathrm{CM}$ & Beiromvand et al. [279] \\
\hline Iran & 2280 & $81(3.6)$ & NA & $\mathrm{CM}$ & Taherkhani et al. [280] \\
\hline Iraq & 78 & $1(1.3)$ & NA & CM, IVC & Sayal et al. [148] \\
\hline Korea & 324 & $29(9.0)$ & ST1, ST2, ST3 & MOL & Kim et al. [281] \\
\hline Laos & 669 & $91(13.6)$ & NA & $\mathrm{CM}$ & Sayasone et al. [282] \\
\hline Laos & 305 & $45(14.8)$ & NA & $\mathrm{CM}$ & Ribas et al. [283] \\
\hline Laos & 60 & $32(51.7)$ & ST1, ST2, ST3, ST7 & CM, IVC, MOL & Sanpool et al. [284] \\
\hline Lebanon & 7477 & $178(2.3)$ & NA & $\mathrm{CM}$ & Araj et al. [285] \\
\hline Lebanon & 306 & $195(63.7)$ & ST1, ST2, ST3, ST10 & MOL & Khaled et al. [286] \\
\hline Malaysia & 77 & $4(5.2)$ & NA & $\mathrm{CM}$ & Sinniah et al. [287] \\
\hline Malaysia & 500 & $102(20.4)$ & NA & $\mathrm{CM}$ & Anuar et al. [17] \\
\hline Malaysia & 243 & $45(18.5)$ & ST1, ST2, ST3 & MOL & Mohammad et al. [288] \\
\hline Malaysia & 466 & $191(41.0)$ & NA & CM, IVC, MOL & Noradilah et al. [289] \\
\hline Malaysia & 253 & $103(40.7)$ & NA & IVC & Mohammad et al. [290] \\
\hline Malaysia & 473 & $191(40.4)$ & ST1, ST2, ST3, ST4 & MOL & Noradilah et al. [291] \\
\hline Malaysia & 466 & $191(41.0)$ & NA & CM, IVC, MOL & Noradilah et al. [292] \\
\hline Malaysia & 253 & $45(17.8)$ & ST1, ST2, ST3 & MOL & Mohammad et al. [293] \\
\hline Malaysia & 416 & $18(4.3)$ & NA & $\mathrm{CM}$ & Muslim et al. [294] \\
\hline Myanmar & 172 & $16(9.3)$ & ST1, ST3, ST4 & MOL & Gong et al. [248] \\
\hline Nepal & 241 & $63(26.1)$ & ST1, ST2, ST4 & IVC, MOL & Lee et al. [295] \\
\hline Philippines & 110 & $36(32.7)$ & NA & IVC & Santos and Rivera [296] \\
\hline Philippines & 1271 & $166(13.0)$ & $\begin{array}{c}\mathrm{ST} 1, \mathrm{ST} 2, \mathrm{ST} 3, \mathrm{ST} 4 \\
\text { ST5 }\end{array}$ & IVC, MOL & Belleza et al. [297] \\
\hline Philippines & 35 & $29(82.9)$ & ST1, ST3, ST4 & MOL & Adao et al. [298] \\
\hline Philippines & 1271 & $165(13.0)$ & NA & IVC & Belleza et al. [299] \\
\hline Philippines & 412 & $242(58.7)$ & NA & MOL & Weerakoon et al. [300] \\
\hline $\begin{array}{l}\text { Saudi } \\
\text { Arabia }\end{array}$ & 140 & $96(68.6)$ & NA & $\mathrm{CM}$ & AlDahhasi et al. [301] \\
\hline $\begin{array}{l}\text { Saudi } \\
\text { Arabia }\end{array}$ & 80 & $12(15.0)$ & ST1, ST2, ST5 & MOL & Mohamed et al. [90] \\
\hline $\begin{array}{l}\text { Saudi } \\
\text { Arabia }\end{array}$ & 50 & $4(8.0)$ & NA & $\mathrm{CM}$ & Hawash et al. [302] \\
\hline $\begin{array}{l}\text { Saudi } \\
\text { Arabia }\end{array}$ & 90 & $2(2.2)$ & NA & $\mathrm{CM}$ & Hawash et al. [159] \\
\hline $\begin{array}{l}\text { Saudi } \\
\text { Arabia }\end{array}$ & 795 & $131(16.5)$ & NA & $\mathrm{CM}$ & Alqumber [303] \\
\hline $\begin{array}{l}\text { Saudi } \\
\text { Arabia }\end{array}$ & 795 & $209(26.3)$ & NA & $\mathrm{CM}$ & Alqumber [303] \\
\hline Thailand & 249 & $1(0.4)$ & NA & $\mathrm{CM}$ & Kaewpitoon et al. [304] \\
\hline Thailand & 60 & $6(10.0)$ & NA & IVC & Surangsrirat et al. [149] \\
\hline Thailand & 475 & $58(12.2)$ & NA & CM, IVC & Kaewjai et al. [305] \\
\hline
\end{tabular}


Table 7. Cont.

\begin{tabular}{|c|c|c|c|c|c|}
\hline Country & $\begin{array}{l}\text { No. of Samples } \\
\text { Examined }\end{array}$ & $\begin{array}{c}\text { No. of Positive } \\
\text { Samples (\%) }\end{array}$ & $\begin{array}{l}\text { Subtypes (STs) } \\
\text { Identified }\end{array}$ & Method(s) & References \\
\hline Thailand & 230 & $25(10.8)$ & ST1, ST3, ST4 & MOL & Popruk et al. [306] \\
\hline Thailand & 1047 & $29(2.8)$ & NA & $\mathrm{CM}$ & Prommi et al. [307] \\
\hline Thailand & 178 & $41(23.0)$ & $\begin{array}{l}\text { ST1, ST2 ST3, ST4, } \\
\text { ST6, ST7 }\end{array}$ & MOL & Yowang et al. [308] \\
\hline Thailand & 324 & $13(4.0)$ & NA & $\mathrm{CM}$ & Punsawad et al. [309] \\
\hline Thailand & 220 & $13(5.9)$ & ST2, ST3, ST6 & MOL & Palasuwan et al. [310] \\
\hline Thailand & 247 & $2(0.8)$ & NA & $\mathrm{CM}$ & Kitvatanachai et al. [311] \\
\hline Thailand & 253 & $4(1.6)$ & NA & $\mathrm{CM}$ & Boonjaraspinyo et al. [312] \\
\hline Thailand & 224 & $1(0.4)$ & NA & $\mathrm{CM}$ & $\begin{array}{l}\text { Suntaravitun and } \\
\text { Dokmaikaw [313] }\end{array}$ \\
\hline Thailand & 733 & $57(7.8)$ & NA & IVC & Wongthamarin et al. [314] \\
\hline Thailand & 207 & $77(37.2)$ & ST1, ST2, ST3, ST4 & MOL & Popruk et al. [315] \\
\hline Turkey & 30 & $4(13.0)$ & NA & $\mathrm{CM}, \mathrm{MOL}$ & Karasartova et al. [316] \\
\hline Turkey & 150 & $16(10.7)$ & NA & $\mathrm{CM}$ & Karadag et al. [317] \\
\hline Turkey & 105 & $30(28.6)$ & NA & IVC & Dogruman-Al et al. [318] \\
\hline Turkey & 27,664 & $581(2.1)$ & NA & $\mathrm{CM}$ & Koksal et al. [319] \\
\hline Turkey & 176 & $30(17.0)$ & NA & $\mathrm{CM}$ & Alver et al. [160] \\
\hline Turkey & 16,445 & $2602(15.8)$ & NA & $\mathrm{CM}$ & Çetinkaya et al. [320] \\
\hline Turkey & 17,711 & $1353(7.6)$ & NA & $\mathrm{CM}$ & Düzyol et al. [321] \\
\hline Turkey & 251 & $54(21.5)$ & NA & $\mathrm{CM}$ & Kurt et al. [322] \\
\hline Turkey & 6267 & $968(15.4)$ & NA & $\mathrm{CM}$ & Y1lmaz et al. [323] \\
\hline Turkey & 87,100 & $640(0.7)$ & NA & & Gülmez et al. [324] \\
\hline Turkey & 111,889 & $306(0.3)$ & NA & $\mathrm{CM}$ & Kirkoyun Uysal et al. [325] \\
\hline Turkey & 7353 & $1884(63.6)$ & NA & $\mathrm{CM}$ & Öncel [326] \\
\hline Turkey & 200 & $93(46.5)$ & ST1, ST2, ST3, ST7 & MOL & Malatyalı et al. [327] \\
\hline Turkey & 69,633 & $18,460(26.5)$ & NA & $\mathrm{CM}$ & Taş Cengiz et al. [328] \\
\hline Turkey & 104 & $10(9.6)$ & ST1, ST2, ST3, ST6 & MOL & Gulhan et al. [329] \\
\hline Turkey & 56 & $28(50.0)$ & $\begin{array}{l}\text { ST1, ST2, ST3, ST4 } \\
\text { ST5, ST6, ST7 }\end{array}$ & MOL & Koltas and Eroglu [330] \\
\hline $\begin{array}{l}\text { United Arab } \\
\text { Emirates }\end{array}$ & 133 & $59(44.4)$ & ST1, ST2, ST3 & MOL & AbuOdeh et al. [331] \\
\hline Uzbekistan & 300 & $31(10.3)$ & NA & $\mathrm{CM}$ & Toychiev et al. [332] \\
\hline Uzbekistan & 550 & $99(18.0)$ & NA & $\mathrm{CM}$ & Davis et al. [112] \\
\hline
\end{tabular}

$\mathrm{CM}-$ Conventional microscopy, IVC - In vitro cultivation, MOL-Molecular technique, NA-Not applicable.

The presence of Blastocystis spp. in various other human categories that do not quite fit into those discussed above is summarized in Table 8.

Table 8. Prevalence and subtype distribution of Blastocystis spp. in various human categories in Asia (2010-2021).

\begin{tabular}{|c|c|c|c|c|c|c|}
\hline Host & Country & $\begin{array}{l}\text { No. of Samples } \\
\text { Examined }\end{array}$ & $\begin{array}{l}\text { No. of Positive } \\
\text { Samples (\%) }\end{array}$ & $\begin{array}{l}\text { Subtypes (STs) } \\
\text { Identified }\end{array}$ & Method(s) & References \\
\hline $\begin{array}{l}\text { Acute appendicitis } \\
\text { patients }\end{array}$ & Turkey & 136 & $8(5.9)$ & NA & $\mathrm{CM}$ & Hatipoğlu et al. [333] \\
\hline $\begin{array}{l}\text { Adult male prison } \\
\text { inmates }\end{array}$ & Malaysia & 294 & 43 (14.6) & ST1, ST3, ST6 & $\mathrm{CM}, \mathrm{IVC}, \mathrm{MOL}$ & Angal et al. [334] \\
\hline $\begin{array}{l}\text { Adults with intestinal } \\
\text { parasitic infection }\end{array}$ & Malaysia & 35 & $17(48.0)$ & NA & IVC & $\begin{array}{c}\text { Chandramathi et al. } \\
\text { [335] }\end{array}$ \\
\hline $\begin{array}{l}\text { Asymptomatic } \\
\text { Blastocystis positive } \\
\text { patients }\end{array}$ & Iran & $25^{*}$ & 25 & $\begin{array}{l}\text { ST1, ST2, ST3, } \\
\text { ST7 }\end{array}$ & MOL & Rezaei Riabi et al. [336] \\
\hline $\begin{array}{l}\text { Asymptomatic } \\
\text { Blastocystis positive } \\
\text { patients }\end{array}$ & Iran & $34 *$ & 34 & ST2, ST3 & CM, MOL & Shahbazi et al. [142] \\
\hline $\begin{array}{l}\text { Chronic spontaneous } \\
\text { urticaria (adults) }\end{array}$ & Turkey & 38 & $7(18.4)$ & NA & $\mathrm{CM}$ & Vezir et al. [337] \\
\hline
\end{tabular}


Table 8. Cont.

\begin{tabular}{|c|c|c|c|c|c|c|}
\hline Host & Country & $\begin{array}{l}\text { No. of Samples } \\
\text { Examined }\end{array}$ & $\begin{array}{l}\text { No. of Positive } \\
\text { Samples (\%) }\end{array}$ & $\begin{array}{l}\text { Subtypes (STs) } \\
\text { Identified }\end{array}$ & Method(s) & References \\
\hline $\begin{array}{c}\text { Chronic spontaneous } \\
\text { urticaria (children) }\end{array}$ & Turkey & 76 & $13(17.1)$ & NA & $\mathrm{CM}$ & Vezir et al. [337] \\
\hline Urticarial patients & Turkey & 133 & $16(12.0)$ & ST1, ST2, ST3 & $\mathrm{CM}, \mathrm{MOL}$ & Aydin et al. [338] \\
\hline Cirrhotic patients & Turkey & 37 & $8(21.6)$ & ST1, ST2, ST3 & MOL & Yildiz et al. [339] \\
\hline $\begin{array}{c}\text { Diarrheic and } \\
\text { non-diarrheic patients }\end{array}$ & Iran & 400 & $58(14.5)$ & ST1, ST2, ST3 & IVC, MOL & $\begin{array}{c}\text { Alinaghizade et al. } \\
\text { [340] }\end{array}$ \\
\hline Dengue patients & Malaysia & 89 & $21(23.6)$ & $\begin{array}{c}\text { ST1, ST3, ST4, } \\
\text { ST6 }\end{array}$ & IVC, MOL & Thergarajan et al. [341] \\
\hline Dialysis patients & Turkey & 142 & $34(23.9)$ & NA & $\mathrm{CM}$ & Karadag et al. [317] \\
\hline $\begin{array}{l}\text { Giardia intestinalis } \\
\text { positive patients }\end{array}$ & India & 258 & $21(8.1)$ & NA & $\mathrm{CM}$ & Roy et al. [342] \\
\hline $\begin{array}{c}\text { Hemodialysis } \\
\text { patients }\end{array}$ & Iran & 88 & $8(9.0)$ & NA & $\mathrm{CM}$ & Barazesh et al. [343] \\
\hline $\begin{array}{l}\text { Immunocompromised } \\
\text { and control }\end{array}$ & Iran & 641 & $57(8.9)$ & NA & $\mathrm{CM}$ & Mahmoudi et al. [259] \\
\hline $\begin{array}{c}\text { Orphanage } \\
\text { (orphansand } \\
\text { childcare workers) }\end{array}$ & Thailand & 343 & $94(27.4)$ & NA & CM, IVC & $\begin{array}{l}\text { Pipatsatitpong et al. } \\
\text { [344] }\end{array}$ \\
\hline $\begin{array}{c}\text { Patients suspected to } \\
\text { have } \\
\text { intestinalparasites }\end{array}$ & Turkey & 918 & $38(4.2)$ & NA & $\mathrm{CM}$ & Koltas et al. [345] \\
\hline $\begin{array}{l}\text { Patients with chronic } \\
\text { renal failure }\end{array}$ & Saudi Arabia & 50 & $8(16.0)$ & NA & $\mathrm{CM}$ & Hawash et al. [302] \\
\hline $\begin{array}{l}\text { Patients with chronic } \\
\text { viral Hepatitis C }\end{array}$ & Russia & 327 & $108(33.0)$ & ST3, ST5, ST6 & $\mathrm{CM}, \mathrm{MOL}$ & Sigidaev et al. [134] \\
\hline $\begin{array}{l}\text { Patients with } \\
\text { Erythema Nodosum }\end{array}$ & Turkey & 81 & $2(2.5)$ & NA & & Ozbagcivan et al. [346] \\
\hline $\begin{array}{l}\text { Patients with } \\
\text { gastrointestinal } \\
\text { and/or dermatologic } \\
\text { symptoms }\end{array}$ & Turkey & 37,108 & $2537(6.8)$ & NA & $\mathrm{CM}$ & Tunalı et al. [347] \\
\hline $\begin{array}{l}\text { Patients with } \\
\text { intestinal protozoan } \\
\text { infections }\end{array}$ & Iran & 75 & $5(6.7)$ & NA & $\mathrm{CM}$ & Jafari et al. [348] \\
\hline $\begin{array}{l}\text { Patients with systemic } \\
\text { lupus erythematosus } \\
\text { (SLE) }\end{array}$ & Malaysia & 187 & $1(0.5)$ & NA & not stated & Teh et al. 2018 [349] \\
\hline $\begin{array}{l}\text { Post-traumatic } \\
\text { splenectomized } \\
\text { patients }\end{array}$ & Turkey & 30 & $12(40.0)$ & ST1, ST3 & $\mathrm{CM}, \mathrm{MOL}$ & Karasartova et al. [316] \\
\hline Pregnant women & Turkey & 100 & $14(14.0)$ & ST1, ST2, ST3 & $\mathrm{CM}, \mathrm{IVC}, \mathrm{MOL}$ & Malatyalı et al. [350] \\
\hline $\begin{array}{c}\text { Symptomatic } \\
\text { Blastocystis positive } \\
\text { patients }\end{array}$ & Iran & $30 *$ & 30 & $\begin{array}{l}\text { ST1, ST2, ST3, } \\
\text { ST6 }\end{array}$ & MOL & Rezaei Riabi et al. [336] \\
\hline $\begin{array}{l}\text { Ulcerative colitis } \\
\text { patients with } \\
\text { refractory symptoms }\end{array}$ & China & 49 & $6(12.2)$ & NA & $\mathrm{CM}$ & Tai et al. [351] \\
\hline $\begin{array}{l}\text { Ulcerative colitis } \\
\text { patients responsive to } \\
\text { treatment }\end{array}$ & China & 73 & $1(1.4)$ & NA & $\mathrm{CM}$ & Tai et al. [351] \\
\hline $\begin{array}{c}\text { Visceral } \\
\text { Leishmaniasis cases }\end{array}$ & India & 23 & $14(60.9)$ & NA & MOL & Lappan et al. [253] \\
\hline
\end{tabular}

* Study was carried out on Blastocystis spp. positive hosts, CM-Conventional microscopy, IVC—In vitro cultivation, MOL-Molecular technique, NA-Not applicable.

\section{Blastocystis spp. Infection in Animals}

In Asia, Blastocystis spp. infection have been documented in hoofed mammals (Table 9), carnivores (Table 10), non-human primates (NHPs) (Table 11), birds (Table 12), rodents (Table 13), reptiles (Table 14), insects and some other mammalian groups (Table 15). 
Table 9. Prevalence and subtype distribution of Blastocystis spp. in ungulates in Asia (2010-2021).

\begin{tabular}{|c|c|c|c|c|c|c|}
\hline Host & Country & $\begin{array}{l}\text { No. of Samples } \\
\text { Examined }\end{array}$ & $\begin{array}{l}\text { No. of Positive } \\
\text { Samples (\%) }\end{array}$ & $\begin{array}{l}\text { Subtypes (STs) } \\
\text { Identified }\end{array}$ & Method(s) & References \\
\hline \multicolumn{7}{|l|}{ Artiodactyla } \\
\hline Alpaca & China & 14 & $12(85.7)$ & ST10, ST14, ST18 & MOL & Zhao et al. [352] \\
\hline Alpaca & China & 27 & $4(14.8)$ & ST10, ST14 & MOL & Li et al. [353] \\
\hline Alpaca & China & 366 & $87(23.8)$ & ST5, ST10, ST14 & MOL & Ma et al. [354] \\
\hline Alpaca & China & 11 & $4(36.4)$ & ST10, ST14 & MOL & Deng et al. [3] \\
\hline Blesbuck & China & 2 & $1(50.0)$ & ST5 & MOL & Li et al. [353] \\
\hline Buffalo & India & 1 & $1(100.0)$ & NA & $\mathrm{CM}$ & Sreekumar et al. [355] \\
\hline Buffalo & Nepal & 19 & $4(21.1)$ & ST4 & IVC, MOL & Lee et al. [18] \\
\hline Bushbuck & China & 18 & $8(61.5)$ & ST10, ST14 & MOL & Zhao et al. [352] \\
\hline Camel & China & 10 & $5(50.0)$ & ST1, ST10 & MOL & Zhao et al. [352] \\
\hline Camel & China & 40 & $14(35.0)$ & $\begin{array}{l}\text { ST2, ST10, ST14 } \\
\text { ST1, ST2, ST3, }\end{array}$ & MOL & Zhang et al. [14] \\
\hline Cattle & Lebanon & 254 & $161(63.4)$ & $\begin{array}{l}\text { ST5, ST7, ST10, } \\
\text { ST14 }\end{array}$ & MOL & Greige et al. [197] \\
\hline Cattle & Malaysia & 29 & $10(34.5)$ & NA & IVC & Hemalatha et al. [356] \\
\hline Cattle & Malaysia & 3 & $1(33.3)$ & ST10 & MOL & Mohammad et al. [288] \\
\hline Cattle & Malaysia & 110 & $6(5.4)$ & NA & IVC & Abd Razak et al. [357] \\
\hline Cattle & Malaysia & 80 & $35(43.8)$ & $\begin{array}{c}\text { ST1, ST3, ST4, } \\
\text { ST5, ST10, ST14 }\end{array}$ & MOL & Kamaruddin et al. [358] \\
\hline Cattle & Nepal & 6 & $1(16.7)$ & Unknown & IVC, MOL & Lee et al. [18] \\
\hline Cattle & Thailand & 42 & $21(50.0)$ & ST10, ST12 & MOL & Udonsom et al. [215] \\
\hline Cattle & Turkey & 80 & $9(11.3)$ & ST10, ST14 & MOL & Aynur et al. [359] \\
\hline Cattle & Indonesia & 500 & $72(14.4)$ & NA & $\mathrm{CM}$ & Hastutiek et al. [360] \\
\hline Cattle & Indonesia & 100 & $100(100.0)$ & NA & $\mathrm{CM}$ & Susana et al. [361] \\
\hline Cattle & Indonesia & 108 & 108 (100.0) & ST10 & CM, IVC, MOL & Suwanti et al. [362] \\
\hline Cattle & Iran & 198 & $19(9.6)$ & ST3, ST5, ST6 & MOL & Badparva et al. [363] \\
\hline Cattle & Iran & 75 & $25(33.3)$ & ST5, ST10 & CM, MOL & Sharifi et al. [195] \\
\hline Cattle & Iran & 40 & $14(35.0)$ & ST3, ST10, ST14 & CM, MOL & Rostami et al. [364] \\
\hline Cattle & Japan & 133 & $72(54.1)$ & ST10, ST14 & MOL & Masuda et al. [365] \\
\hline Cattle & China & 526 & $54(10.3)$ & $\begin{array}{c}\text { ST4, ST5, ST10, } \\
\text { ST14 }\end{array}$ & MOL & Zhu et al. [366] \\
\hline Cattle & China & 147 & $14(9.5)$ & ST3, ST10, ST14 & MOL & Wang et al. [367] \\
\hline Cattle & China & 57 & $15(26.3)$ & ST10, ST14 & MOL & Zhang et al. [14] \\
\hline Cattle & Korea & 1512 & $101(6.7)$ & $\begin{array}{l}\text { ST1, ST5, ST10, } \\
\text { ST14 }\end{array}$ & MOL & Lee et al. [368] \\
\hline Cattle & $\begin{array}{l}\text { United Arab } \\
\text { Emirates }\end{array}$ & 22 & $5(22.7)$ & ST10 & MOL & AbuOdeh et al. [369] \\
\hline $\begin{array}{c}\text { Deer (Caspian red } \\
\text { deer) }\end{array}$ & Iran & 1 & $1(100.0)$ & NA & $\mathrm{CM}$ & Mirzapour et al. [370] \\
\hline Deer (Javan rusa) & Malaysia & 50 & $14(28.0)$ & ST10 & MOL & Mohammad et al. [371] \\
\hline Deer (Mousedeer) & Malaysia & 4 & $1(25.0)$ & $\begin{array}{l}\text { Unknown (Clade } \\
\text { IV) }\end{array}$ & IVC, MOL & Mohd Zain et al. [372] \\
\hline Deer (Sambar deer) & Malaysia & 14 & $4(28.6)$ & NA & IVC & Hemalatha et al. [356] \\
\hline Deer (Sika deer) & Malaysia & 50 & $16(32.0)$ & ST10 & MOL & Mohammad et al. [371] \\
\hline Deer (Red deer) & China & 5 & $2(40.0)$ & ST10 & MOL & Li et al. [353] \\
\hline $\begin{array}{c}\text { Deer (Red } \\
\text { deer/Wapiti) }\end{array}$ & China & 3 & $1(33.3)$ & ST10 & MOL & Zhao et al. [352] \\
\hline Deer (Reindeer) & China & 104 & $7(6.7)$ & ST10, ST13 & MOL & Wang et al. [373] \\
\hline Deer (Fallow deer) & China & 2 & $1(50.0)$ & ST10 & MOL & Zhao et al. [352] \\
\hline $\begin{array}{l}\text { Deer (White-lipped } \\
\text { deer) }\end{array}$ & China & 1 & $1(100.0)$ & ST10 & MOL & Zhao et al. [352] \\
\hline Deer (Sika deer) & China & 8 & $3(37.5)$ & ST10 & MOL & Zhao et al. [352] \\
\hline Deer (Sika deer) & China & 82 & $12(14.6)$ & ST10, ST14 & MOL & Wang et al. [373] \\
\hline Deer (Sika deer) & China & 11 & $1(9.1)$ & ST1 & MOL & Deng et al. [3] \\
\hline Deer (Sika deer) & China & 760 & $6(0.8)$ & ST10, ST14 & MOL & Ni et al. [374] \\
\hline Deer (Spotted deer) & Bangladesh & 30 & $1(3.3)$ & ST14 & MOL & Li et al. [375] \\
\hline Deer (Water deer) & Korea & 125 & $51(40.8)$ & ST4, ST14 & MOL & Kim et al. [376] \\
\hline Eland & China & 9 & $6(66.7)$ & ST10, ST14 & MOL & Zhao et al. [352] \\
\hline Gayal & Bangladesh & 4 & $1(25.0)$ & ST14 & MOL & Li et al. [375] \\
\hline Giraffe & China & 10 & $2(20.0)$ & ST12 & MOL & Zhao et al. [352] \\
\hline Goat & China & 789 & $458(58.0)$ & $\begin{array}{c}\text { ST1, ST3, ST4, } \\
\text { ST5, ST10, ST14 }\end{array}$ & MOL & Song et al. [377] \\
\hline Goat & China & 781 & $2(0.3)$ & ST1 & MOL & Li et al. [378] \\
\hline Goat & China & 59 & $28(47.5)$ & ST10, ST14 & MOL & Zhang et al. [14] \\
\hline Goat & Nepal & 400 & $3(0.8)$ & NA & $\mathrm{CM}$ & $\begin{array}{c}\text { Ghimire and Bhattarai } \\
\text { [379] }\end{array}$ \\
\hline
\end{tabular}


Table 9. Cont.

\begin{tabular}{|c|c|c|c|c|c|c|}
\hline Host & Country & $\begin{array}{l}\text { No. of Samples } \\
\text { Examined }\end{array}$ & $\begin{array}{l}\text { No. of Positive } \\
\text { Samples (\%) }\end{array}$ & $\begin{array}{l}\text { Subtypes (STs) } \\
\text { Identified }\end{array}$ & Method(s) & References \\
\hline Goat & Malaysia & 236 & $73(30.9)$ & ST1, ST3, ST6, ST7 & MOL & Tan et al. [380] \\
\hline Goat & Malaysia & 31 & $8(25.8)$ & ST4, ST8, ST10 & MOL & Noradilah et al. [15] \\
\hline Goat & Malaysia & 65 & $14(21.5)$ & NA & IVC & Abd Razak et al. [357] \\
\hline Goat & Malaysia & 20 & $13(65.0)$ & NA & IVC & Hemalatha et al. [356] \\
\hline Goat & Nepal & 29 & $1(3.4)$ & ST4 & IVC, MOL & Lee et al. [18] \\
\hline Goat & Philippines & 6 & $1(16.7)$ & ST14 & IVC, MOL & Adao et al. [381] \\
\hline Goat & Thailand & 38 & $36(94.7)$ & ST10, ST12, ST14 & MOL & Udonsom et al. [215] \\
\hline Goral (Himalayan) & Nepal & 19 & $1(5.3)$ & NA & $\mathrm{CM}$ & Adhikari et al. [382] \\
\hline Guanaco & China & 20 & $14(70.0)$ & ST10, ST22 & MOL & Zhao et al. [352] \\
\hline Guar & Malaysia & 10 & $3(30.0)$ & NA & IVC & Hemalatha et al. [356] \\
\hline Oryx & China & 2 & $1(50.0)$ & ST10 & MOL & Zhao et al. [352] \\
\hline Oryx & China & 11 & $1(9.1)$ & ST5 & MOL & Li et al. [353] \\
\hline Pig & Cambodia & 73 & $33(45.2)$ & ST5 & MOL & Wang et al. [242] \\
\hline Pig & China & 560 & $419(74.8)$ & $\begin{array}{c}\text { ST1, ST3, ST5 } \\
\text { ST10 }\end{array}$ & MOL & Song et al. [383] \\
\hline Pig & China & 68 & $6(8.8)$ & ST5 & MOL & Wang et al. [367] \\
\hline Pig & China & 801 & $174(21.7)$ & ST1, ST3, ST5 & MOL & Wang et al. [384] \\
\hline Pig & China & 866 & $433(50.0)$ & ST1, ST3, ST5 & MOL & Han et al. [385] \\
\hline Pig & China & 396 & $170(42.9)$ & ST1, ST5 & MOL & Zou et al. [386] \\
\hline Pig & India & 1 & $1(100.0)$ & NA & $\mathrm{CM}$ & Sreekumar et al. [355] \\
\hline Pig & India & 90 & $85(94.4)$ & NA & $\mathrm{CM}$ & Arpitha et al. [387] \\
\hline Pig & Indonesia & 93 & $81(87.1)$ & ST1, ST2, ST5, ST7 & IVC, MOL & Yoshikawa et al. [41] \\
\hline Pig & Indonesia & 100 & $63(63.0)$ & NA & CM & Mahendra et.al. [388] \\
\hline Pig & Indonesia & 100 & $69(69.0)$ & NA & CM & Widisuputri et al. [389] \\
\hline Pig & Korea & 646 & $390(60.4)$ & ST1, ST2, ST3, ST5 & MOL & Paik et al. [390] \\
\hline Pig & Nepal & 11 & $4(36.4)$ & ST4 & IVC, MOL & Lee et al. [18] \\
\hline Pig & Philippines & 49 & $36(73.5)$ & ST1, ST2, ST3, ST5 & MOL & Adao et al. [391] \\
\hline Pig & Philippines & 99 & $20(20.2)$ & ST1, ST5, ST7 & IVC, MOL & Adao et al. [381] \\
\hline Pig & Philippines & 122 & $47(38.5)$ & NA & CM, IVC & De La Cruz et al. [392] \\
\hline Pig & Philippines & 100 & $14(14.0)$ & ST1, ST5 & IVC, MOL & Evidor and Rivera [393] \\
\hline Pig & Philippines & 101 & $2(2.0)$ & NA & $\mathrm{CM}$ & Murao et al. [394] \\
\hline Pig & Thailand & 102 & $32(31.4)$ & $\begin{array}{c}\text { ST1, ST3, ST12, } \\
\text { ST14 }\end{array}$ & MOL & Sanyanusin et al. [395] \\
\hline Pig & Thailand & 90 & $32(35.6)$ & ST1, ST3, ST5 & MOL & Pintong et al. [214] \\
\hline Pig & Thailand & 87 & $40(46.0)$ & ST1, ST5 & MOL & Udonsom et al. [215] \\
\hline Pig & Malaysia & 10 & $10(100.0)$ & NA & IVC & Hemalatha et al. [356] \\
\hline Pig & Vietnam & 12 & $12(100.0)$ & ST5 & MOL & Alfellani et al. [396] \\
\hline Sheep & Iran & 150 & $29(19.3)$ & ST7, ST10 & CM, MOL & Rostami et al. [364] \\
\hline Sheep & China & 832 & $50(6.0)$ & ST5, ST10, ST14 & MOL & Li et al. [378] \\
\hline Sheep & China & 109 & $6(5.5)$ & $\begin{array}{c}\text { ST1, ST5, ST10, } \\
\text { ST14 }\end{array}$ & MOL & Wang et al. [367] \\
\hline Sheep & China & 38 & $16(42.1)$ & ST2, ST10, ST14 & MOL & Zhang et al. [14] \\
\hline Sheep & China & 78 & $42(53.8)$ & ST2, ST10, ST14 & MOL & Zhang et al. [14] \\
\hline Sheep & $\begin{array}{l}\text { United Arab } \\
\text { Emirates }\end{array}$ & 11 & $7(63.6)$ & ST10, ST14 & MOL & AbuOdeh et al. [369] \\
\hline Sheep & Malaysia & 38 & $22(57.9)$ & NA & IVC & Hemalatha et al. [356] \\
\hline Sheep & Malaysia & 20 & $2(10.0)$ & NA & IVC & Abd Razak et al. [357] \\
\hline Small ruminants & India & 107 & $15(14.0)$ & NA & $\mathrm{CM}$ & Arpitha et al. [387] \\
\hline Takin & China & 49 & $28(57.1)$ & ST10, ST12, ST14 & MOL & Zhao et al. [352] \\
\hline Waterbuck & China & 3 & $3(100.0)$ & ST12, ST14 & MOL & Zhao et al. [352] \\
\hline Waterbuck & China & 2 & $1(50.0)$ & ST21 & MOL & Zhao et al. [352] \\
\hline Waterbuck & Bangladesh & 7 & $1(14.3)$ & ST10 & MOL & Li et al. [375] \\
\hline Wild boar & South Korea & 433 & $45(10.4)$ & ST5 & MOL & Lee et al. [397] \\
\hline Wild Boar & Iran & 25 & $11(44.0)$ & NA & $\mathrm{CM}$ & Yaghoobi et al. [398] \\
\hline Wild Boar & Iran & 1 & $1(100.0)$ & NA & CM & Mirzapour et al. [370] \\
\hline Yak & China & 1027 & $278(27.1)$ & ST10, ST12, ST14 & MOL & Ren et al. [399] \\
\hline Yak & China & 102 & $39(38.2)$ & ST2, ST10, ST14 & MOL & Zhang et al. [14] \\
\hline Yak & China & 6 & $3(50.0)$ & ST10, ST14 & MOL & Zhao et al. [352] \\
\hline \multicolumn{7}{|l|}{ Perissodactyla } \\
\hline Horse & China & 32 & $9(28.1)$ & ST2, ST10 & MOL & Zhang et al. [14] \\
\hline Horse & China & 4 & $1(25.0)$ & ST10 & MOL & Zhao et al. [352] \\
\hline Wild Ass & China & 5 & $2(40.0)$ & ST10, ST12 & MOL & Zhao et al. [352] \\
\hline Pony & China & 6 & $1(16.7)$ & ST10 & MOL & Zhao et al. [352] \\
\hline Zebra & China & 7 & $1(14.3)$ & ST10 & MOL & Li et al. [353] \\
\hline
\end{tabular}


Table 9. Cont

\begin{tabular}{ccccccc}
\hline Host & Country & $\begin{array}{c}\text { No. of Samples } \\
\text { Examined }\end{array}$ & $\begin{array}{c}\text { No. of Positive } \\
\text { Samples (\%) }\end{array}$ & $\begin{array}{c}\text { Subtypes (STs) } \\
\text { Identified }\end{array}$ & Method(s) & References \\
\hline $\begin{array}{c}\text { Proboscidea } \\
\text { Elephant }\end{array}$ & Bangladesh & 3 & $1(33.3)$ & ST11 & MOL & Li et al. [375] \\
\hline
\end{tabular}

CM-Conventional microscopy, IVC—In vitro cultivation, MOL—Molecular technique, NA—Not applicable.

Table 10. Prevalence and subtype distribution of Blastocystis spp. in carnivorous animals in Asia (2010-2021).

\begin{tabular}{|c|c|c|c|c|c|c|}
\hline Host & Country & $\begin{array}{l}\text { No. of Samples } \\
\text { Examined }\end{array}$ & $\begin{array}{l}\text { Number of Positive } \\
\text { Samples (\%) }\end{array}$ & $\begin{array}{l}\text { Subtypes (STs) } \\
\text { Identified }\end{array}$ & Method(s) & References \\
\hline Artic fox & China & 213 & $4(1.9)$ & ST1, ST4, ST7 & MOL & Wang et al. [373] \\
\hline Bear & China & 12 & $3(25.0)$ & ST17 & MOL & Deng et al. [3] \\
\hline Bear & China & 312 & 45 (14.4) & ST1 & MOL & $\mathrm{Ni}$ et al. [374] \\
\hline Cat & China & 346 & $2(0.6)$ & ST1 & MOL & Li et al. [400] \\
\hline Cat & Indonesia & 90 & $48(53.3)$ & NA & MOL & Patagi et al. [401] \\
\hline Cat & Iran & 140 & $20(14.3)$ & NA & $\mathrm{CM}$ & Khademvatan et al. [402] \\
\hline Cat & Iran & 119 & $21(17.7)$ & $\begin{array}{l}\text { ST1, ST3, ST4, } \\
\text { ST10, ST14 }\end{array}$ & MOL & $\begin{array}{l}\text { Mohammadpour et al. } \\
\text { [403] }\end{array}$ \\
\hline Cat & $\begin{array}{l}\text { South } \\
\text { Korea }\end{array}$ & 158 & $1(0.6)$ & ST4 & MOL & Kwak and Seo [404] \\
\hline Cat & Malaysia & 60 & $12(20.0)$ & ST1 & MOL & Farah Haziqah et al. \\
\hline Cat & Turkey & 3 & $3(100.0)$ & ST3 & MOL & Eroglu and Koltas [19] \\
\hline Common raccoon & Iran & 30 & $5(6.7)$ & ST1, ST2, ST3 & MOL & $\begin{array}{c}\text { Mohammad Rahimi et al. } \\
\text { [406] }\end{array}$ \\
\hline Dog & China & 136 & $4(2.9)$ & ST1, ST4 & MOL & Wang et al. [373] \\
\hline Dog & China & 651 & $35(5.4)$ & ST1, ST3, ST10 & MOL & Liao et al. [407] \\
\hline Dog & India & 80 & $19(24.0)$ & $\begin{array}{l}\text { ST1, ST4, ST5, } \\
\text { ST6 }\end{array}$ & MOL & Wang et al. [408] \\
\hline Dog & Iran & 301 & $59(19.6)$ & NA & $\mathrm{CM}$ & Mohaghegh et al. [409] \\
\hline Dog & Iran & 552 & $29(5.2)$ & NA & $\mathrm{CM}$ & Mirbadie et al. [410] \\
\hline Dog & Iran & 154 & $29(18.8)$ & $\begin{array}{l}\text { ST2, ST3, ST4, } \\
\text { ST7, ST8, ST10 }\end{array}$ & MOL & $\begin{array}{l}\text { Mohammadpour et al. } \\
\text { [403] }\end{array}$ \\
\hline Dog & Turkey & 4 & $4(100.0)$ & ST1, ST2 & MOL & Eroglu and Koltas [19] \\
\hline Dog & Philippines & 145 & $21(14.5)$ & $\begin{array}{l}\text { ST1, ST2, ST3, } \\
\text { ST4, ST5 }\end{array}$ & IVC, MOL & Belleza et al. [297] \\
\hline Dog & Malaysia & 84 & $40(47.6)$ & $\begin{array}{l}\text { ST1, ST3, ST4, } \\
\text { ST8, ST10 }\end{array}$ & MOL & Noradilah et al. [15] \\
\hline Dog & Thailand & 13 & $1(7.7)$ & ST3 & MOL & Udonsom et al. [215] \\
\hline Dog & Cambodia & 80 & $1(1.3)$ & ST2 & MOL & Wang et al. [408] \\
\hline Dog & China & 7 & $1(14.3)$ & ST10 & MOL & Li et al. [353] \\
\hline Leopard & China & 3 & $2(66.7)$ & ST1, ST5 & MOL & Deng et al. [3] \\
\hline Meerkat & Iran & 1 & $1(100.0)$ & NA & $\mathrm{CM}$ & Mirzapour et al. [370] \\
\hline Meerkat & China & 2 & $1(50.0)$ & ST5 & MOL & Li et al. [353] \\
\hline Panda (Giant panda) & China & 81 & $10(12.3)$ & ST1 & MOL & Deng et al. [411] \\
\hline Panda (Red panda) & China & 23 & $2(8.7)$ & ST1 & MOL & Deng et al. [411] \\
\hline Raccoon dog & China & 40 & $3(7.5)$ & ST3 & MOL & Wang et al. [373] \\
\hline Tiger (Siberian tiger) & China & 13 & $1(7.7)$ & ST10 & MOL & Li et al. [353] \\
\hline Tiger (White tiger) & China & 9 & 1 (11.1) & ST10 & MOL & Li et al. [353] \\
\hline
\end{tabular}

CM-Conventional microscopy, IVC—In vitro cultivation, MOL-Molecular technique, NA—Not applicable. 
Table 11. Prevalence and subtype distribution of Blastocystis spp. in non-human primates in Asia (2010-2021).

\begin{tabular}{|c|c|c|c|c|c|c|}
\hline Host & Country & $\begin{array}{l}\text { No. of Samples } \\
\text { Examined }\end{array}$ & $\begin{array}{c}\text { Number of } \\
\text { Positive Samples } \\
(\%)\end{array}$ & $\begin{array}{l}\text { Subtypes (STs) } \\
\text { Identified }\end{array}$ & Method(s) & References \\
\hline \multicolumn{7}{|l|}{ Primates } \\
\hline Langur & Bangladesh & 5 & $3(60.0)$ & ST1, ST13 & MOL & Li et al. [375] \\
\hline Grey langur & Bangladesh & 2 & $1(50.0)$ & ST1 & MOL & Li et al. [375] \\
\hline $\begin{array}{l}\text { White-cheeked } \\
\text { gibbon }\end{array}$ & China & 4 & $1(25.0)$ & ST1 & MOL & Ma et al. [250] \\
\hline $\begin{array}{l}\text { White-cheeked } \\
\text { gibbon }\end{array}$ & China & 4 & $4(100.0)$ & $\mathrm{ST} 2, \mathrm{ST} 3$ & MOL & Deng et al. [3] \\
\hline Ring-tailed lemur & China & 6 & $2(33.3)$ & ST2, ST4 & MOL & Li et al. [353] \\
\hline Ring-tailed lemur & China & 16 & $7(43.8)$ & ST3, ST5, ST9 & MOL & Ma et al. [250] \\
\hline Ring-tailed lemur & China & 13 & $6(46.2)$ & ST1, ST2 & MOL & Deng et al. [3] \\
\hline Macaque & China & 97 & 85 (87.6) & $\begin{array}{c}\text { ST1, ST2, ST3, } \\
\text { ST5, ST7 }\end{array}$ & MOL & Zanzani et al. [412] \\
\hline Macaque & China & 185 & $12(7.0)$ & ST1, ST2, ST3 & MOL & Zhu et al. [173] \\
\hline $\begin{array}{c}\text { Macaque } \\
\text { (experimental) }\end{array}$ & China & 505 & $235(46.5)$ & $\mathrm{ST} 1, \mathrm{ST} 2, \mathrm{ST} 3$ & MOL & Li et al. [413] \\
\hline Rhesus macaque & Bangladesh & 62 & $20(32.3)$ & ST1, ST2, ST3 & MOL & Li et al. [375] \\
\hline Rhesus macaque & China & 29 & $28(96.6)$ & $\begin{array}{l}\text { ST1, ST2, ST3, } \\
\text { ST19 }\end{array}$ & MOL & Zhao et al. [352] \\
\hline Rhesus macaque & China & 17 & $10(58.8)$ & $\mathrm{ST} 1$ & MOL & Deng et al. [3] \\
\hline Rhesus macaque & China & 18 & $6(33.3)$ & ST2, ST3 & MOL & Ma et al. [250] \\
\hline Japanese macaque & China & 33 & $6(18.2)$ & $\mathrm{ST} 2, \mathrm{ST} 3$ & MOL & Ma et al. [250] \\
\hline Macaque & Philippines & 50 & $5(10.0)$ & NA & $\mathrm{CM}$ & Casim et al. [414] \\
\hline $\begin{array}{l}\text { Long-tailed } \\
\text { macaque }\end{array}$ & Thailand & 628 & $263(41.9)$ & ST1, ST2, ST3 & IVC, MOL & Vaisusuk et al. [415] \\
\hline $\begin{array}{l}\text { Crab-eating } \\
\text { macaque }\end{array}$ & China & 13 & $3(23.1)$ & ST2, ST3 & MOL & Ma et al. [250] \\
\hline Orangutan & Indonesia & 262 & 36 (13.7) & NA & $\mathrm{CM}$ & Labes et al. [416] \\
\hline Orangutan & Malaysia & 10 & $5(50.0)$ & NA & IVC & Hemalatha et al. [356] \\
\hline Vervet monkey & Iran & 40 & $3(7.5)$ & NA & $\mathrm{CM}$ & Dalimi et al. [417] \\
\hline Vervet monkey & Bangladesh & 7 & $3(42.9)$ & ST2, ST3, ST13 & MOL & Li et al. [375] \\
\hline Hamadryas baboon & Saudi Arabia & 823 & $349(42.4)$ & NA & $\mathrm{CM}$ & Alqumber [303] \\
\hline Hamadryas baboon & China & 23 & $13(56.5)$ & ST1, ST3 & MOL & Zhao et al. [352] \\
\hline Chimpanzee & China & 10 & $8(80.0)$ & ST2 & MOL & Zhao et al. [352] \\
\hline Chimpanzee & China & 15 & $3(13.3)$ & ST1, ST5 & MOL & Ma et al. [250] \\
\hline $\begin{array}{l}\text { Francois' leaf } \\
\text { monkey }\end{array}$ & China & 1 & $1(100.0)$ & ST2 & MOL & Zhao et al. [352] \\
\hline $\begin{array}{c}\text { Francois' leaf } \\
\text { monkey }\end{array}$ & China & 3 & $2(66.7)$ & ST1 & MOL & Ma et al. [250] \\
\hline Mandrill & China & 4 & $1(25.0)$ & ST3 & MOL & Zhao et al. [352] \\
\hline Mandrill & China & 15 & $9(60.0)$ & ST1, ST4 & MOL & Ma et al. [250] \\
\hline $\begin{array}{l}\text { De Brazza's } \\
\text { monkey }\end{array}$ & China & 5 & $4(80.0)$ & ST1, ST10 & MOL & Zhao et al. [352] \\
\hline $\begin{array}{c}\text { De Brazza's } \\
\text { monkey }\end{array}$ & China & 5 & $5(100.0)$ & ST1, ST2 & MOL & Ma et al. [250] \\
\hline $\begin{array}{l}\text { Golden snub-nosed } \\
\text { monkey }\end{array}$ & China & 46 & $41(89.1)$ & ST1, ST13 & MOL & Zhao et al. [352] \\
\hline $\begin{array}{c}\text { Snub-nosed } \\
\text { monkey }\end{array}$ & China & 22 & $9(40.9)$ & ST1, ST2 & MOL & Ma et al. [250] \\
\hline Golden monkey & China & 37 & $18(48.6)$ & ST1, ST2, ST3 & MOL & Ma et al. [418] \\
\hline Squirrel monkey & China & 93 & $19(20.4)$ & ST17 & MOL & Deng et al. [3] \\
\hline $\begin{array}{c}\text { Common squirrel } \\
\text { monkey }\end{array}$ & China & 30 & $9(30.0)$ & ST1, ST5 & MOL & Ma et al. [250] \\
\hline $\begin{array}{l}\text { Red-faced spider } \\
\text { monkey }\end{array}$ & China & 4 & $2(50.0)$ & ST2, ST3 & MOL & Ma et al. [250] \\
\hline Monkey & Philippines & 4 & $4(100.0)$ & ST1, ST2, ST3 & MOL & Rivera [21] \\
\hline $\begin{array}{l}\text { Non-human } \\
\text { primates }\end{array}$ & Malaysia & 308 & $5(1.6)$ & NA & $\mathrm{CM}$ & Adrus et al. [419] \\
\hline
\end{tabular}


Table 12. Prevalence and subtype distribution of Blastocystis spp. in birds in Asia (2010-2021).

\begin{tabular}{|c|c|c|c|c|c|c|}
\hline Host & Country & $\begin{array}{l}\text { No. of Samples } \\
\text { Examined }\end{array}$ & $\begin{array}{c}\text { Number of Positive } \\
\text { Samples (\%) }\end{array}$ & $\begin{array}{l}\text { Subtypes (STs) } \\
\text { Identified }\end{array}$ & Method(s) & References \\
\hline Duck & Philippines & 31 & $3(9.6)$ & ST7, B. pythoni & IVC, MOL & Adao et al. [381] \\
\hline Birds & Turkey & 5 & $5(100.0)$ & ST1, ST2 & MOL & Eroglu and Koltas [19] \\
\hline Chicken & China & 46 & $6(13.0)$ & ST6, ST7 & MOL & Wang et al. [373] \\
\hline Chicken & Philippines & 34 & $5(14.7)$ & ST7, Mixed & IVC, MOL & Adao et al. [381] \\
\hline Chicken & India & 24 & $20(83.3)$ & NA & $\mathrm{CM}$ & Sreekumar et al. [355] \\
\hline Chicken & India & 170 & $50(29.4)$ & NA & $\mathrm{CM}$ & Arpitha et al. [387] \\
\hline Chicken & Indonesia & 38 & $13(34.2)$ & ST7 & IVC, MOL & Yoshikawa et al. [41] \\
\hline Chicken & Lebanon & 223 & $71(31.8)$ & ST6, ST7 & MOL & Greige et al. [199] \\
\hline Chicken & Malaysia & 104 & $27(26.0)$ & $\begin{array}{l}\text { ST1, ST3, ST6, } \\
\text { ST7, ST9 }\end{array}$ & MOL & Noradilah et al. [15] \\
\hline Chicken & Malaysia & 15 & $1(6.7)$ & ST6 & MOL & Mohammad et al. [288] \\
\hline Chicken & Malaysia & 107 & $27(25.2)$ & NA & IVC & Farah Haziqah et al. [420] \\
\hline Chicken & Malaysia & 179 & $47(26.3)$ & $\begin{array}{c}\text { ST1, ST6, ST7, } \\
\text { ST8 }\end{array}$ & IVC, MOL & Farah Haziqah et al. [421] \\
\hline Crested ibis & China & 63 & $6(9.5)$ & NA & CM & Zhang et al. [422] \\
\hline Crow (Hooded) & Iran & 144 & $64(44.4)$ & ST13, ST14 & IVC, MOL & Asghari et al. [423] \\
\hline Duck & Malaysia & 20 & $8(40.0)$ & $\begin{array}{c}\text { S11, S12, S13, } \\
\text { ST7 }\end{array}$ & MOL & Noradilah et al. [15] \\
\hline $\begin{array}{l}\text { Green-naped } \\
\text { lorikeet }\end{array}$ & China & 2 & $1(50.0)$ & ST10 & MOL & Li et al. [353] \\
\hline Ostrich & China & 9 & $3(33.3)$ & ST5, ST10, ST20 & MOL & Zhao et al. [352] \\
\hline Ostrich & China & 19 & $6(31.6)$ & ST5 & MOL & Deng et al. [3] \\
\hline Ostrich & Malaysia & 37 & $37(100.0)$ & ST6 & IVC, MOL & Chandrasekaran et al. [424] \\
\hline Ostrich & Malaysia & 37 & $37(100.0)$ & NA & IVC & Hemalatha et al. [424] \\
\hline Ostrich & China & 3 & $2(66.7)$ & ST5 & MOL & Li et al. [353] \\
\hline Green peafowl & China & 12 & $1(8.3)$ & ST3 & MOL & Deng et al. [3] \\
\hline Green peafowl & China & 15 & $1(6.7)$ & ST8 & MOL & Deng et al. [411] \\
\hline Indian peafowl & China & 20 & $3(15.0)$ & ST7, ST8 & MOL & Li et al. [353] \\
\hline Pigeon & China & 34 & $4(11.8)$ & ST8 & MOL & Deng et al. [3] \\
\hline Pigeon & China & 47 & $1(2.1)$ & ST6 & MOL & Wang et al. [373] \\
\hline Pigeon & Iran & 156 & $67(42.9)$ & $\mathrm{ST} 13$ & IVC, MOL & Asghari et al. [423] \\
\hline Poultry & Iran & 132 & $21(15.9)$ & ST7, ST10, ST14 & $\mathrm{CM}, \mathrm{MOL}$ & Rostami et al. [364] \\
\hline $\begin{array}{l}\text { Red crowned } \\
\text { crane }\end{array}$ & China & 43 & $6(14.0)$ & ST6, ST7 & MOL & Wang et al. [373] \\
\hline $\begin{array}{l}\text { Red-crowned } \\
\text { crane }\end{array}$ & China & 2 & $1(50.0)$ & ST14 & MOL & Li et al. [353] \\
\hline Ruddy shelduck & China & 11 & $2(18.2)$ & ST8 & MOL & Deng et al. [411] \\
\hline Swan & Malaysia & 20 & $7(35.0)$ & ST1, ST3 & MOL & Noradilah et al. [15] \\
\hline Black swan & China & 38 & $4(10.5)$ & ST8 & MOL & Deng et al. [411] \\
\hline Turkey & India & 4 & $3(75.0)$ & NA & $\mathrm{CM}$ & Sreekumar et al. [355] \\
\hline
\end{tabular}

Table 13. Prevalence and subtype distribution of Blastocystis spp. in rodents in Asia (2010-2021).

\begin{tabular}{|c|c|c|c|c|c|c|}
\hline Host & Country & $\begin{array}{l}\text { No. of Samples } \\
\text { Examined }\end{array}$ & $\begin{array}{l}\text { Number of Positive } \\
\text { Samples (\%) }\end{array}$ & $\begin{array}{l}\text { Subtypes (STs) } \\
\text { Identified }\end{array}$ & Method(s) & References \\
\hline Flying squirrel & China & 207 & $63(30.4)$ & ST1, ST3, ST13 & MOL & Xiao et al. [425] \\
\hline Eastern chipmunk & China & 171 & $8(4.7)$ & ST4 & MOL & Chai et al. [426] \\
\hline Eurasian red squirrel & China & 72 & $7(9.7)$ & ST4 & MOL & Chai et al. [426] \\
\hline Black great squirrel & China & 1 & $1(100.0)$ & ST4 & MOL & Deng et al. [3] \\
\hline $\begin{array}{l}\text { Red giant flying } \\
\text { squirrel }\end{array}$ & China & 1 & $1(100.0)$ & ST4 & MOL & Deng et al. [3] \\
\hline Indian palm squirrel & $\begin{array}{c}\text { United Arab } \\
\text { Emirates }\end{array}$ & 4 & $2(50.0)$ & ST4 & MOL & AbuOdeh et al. [369] \\
\hline Shrew-faced squirrel & $\begin{array}{l}\text { United Arab } \\
\text { Emirates }\end{array}$ & 1 & $1(100.0)$ & ST17 & MOL & AbuOdeh et al. [369] \\
\hline $\begin{array}{c}\text { Chinese striped } \\
\text { hamster }\end{array}$ & China & 98 & $12(12.2)$ & ST4 & MOL & Chai et al. [426] \\
\hline Chinchilla & China & 72 & $3(4.2)$ & ST4, ST17 & MOL & Chai et al. [426] \\
\hline Chinchilla & China & 6 & $4(66.7)$ & ST17 & MOL & Deng et al. [3] \\
\hline Guinea pig & China & 90 & $12(13.3)$ & ST4 & MOL & Chai et al. [426] \\
\hline Patagonian mara & China & 15 & $3(20.0)$ & ST4 & MOL & Li et al. [353] \\
\hline Rat (Mus musculus) & China & 108 & $4(3.7)$ & ST4 & MOL & Wang et al. [373] \\
\hline
\end{tabular}


Table 13. Cont.

\begin{tabular}{|c|c|c|c|c|c|c|}
\hline Host & Country & $\begin{array}{l}\text { No. of Samples } \\
\text { Examined }\end{array}$ & $\begin{array}{l}\text { Number of Positive } \\
\text { Samples }(\%)\end{array}$ & $\begin{array}{l}\text { Subtypes (STs) } \\
\text { Identified }\end{array}$ & Method(s) & References \\
\hline Laboratory rats & China & 355 & $29(8.2)$ & ST4, ST7 & MOL & Li et al. [427] \\
\hline Rat (Rattus exulans) & Indonesia & 77 & $10(13.0)$ & ST4 & IVC, MOL & Yoshikawa et al. [41] \\
\hline Rat & Indonesia & 98 & $6(6.0)$ & NA & CM & Prasetyo [428] \\
\hline Rat (Rattus exulans) & Indonesia & 67 & $11(16.4)$ & ST4 & MOL & Katsumata et al. [429] \\
\hline Rodents & Iran & 52 & $3(5.8)$ & NA & $\mathrm{CM}$ & Seifollahi et al. [430] \\
\hline Rat (Rattus norvegicus) & Iran & 127 & $20(15.8)$ & ST1, ST3, ST4 & MOL & $\begin{array}{l}\text { Mohammadpour et al. } \\
\text { [403] }\end{array}$ \\
\hline Rat (Rattus norvegicus) & Malaysia & 95 & $48(51.0)$ & NA & $\mathrm{CM}$ & Premaalatha et al. [431] \\
\hline Rat (Rattus norvegicus) & Malaysia & 290 & 133 (45.9) & $\begin{array}{l}\text { ST1, ST4, ST5, } \\
\text { ST7 }\end{array}$ & IVC, MOL & $\begin{array}{c}\text { Farah Haziqah et al. } \\
\text { [432] }\end{array}$ \\
\hline $\begin{array}{c}\text { Wild rats } \\
\text { (Rattus novercious) }\end{array}$ & Japan & 48 & $12(25.0)$ & ST4 & MOL & Katsumata et al. [429] \\
\hline Swiss-Webster mice & Iran & 50 & $1(2.0)$ & NA & $\mathrm{CM}$ & Kalani et al. [433] \\
\hline
\end{tabular}

$\mathrm{CM}-$ Conventional microscopy, IVC—In vitro cultivation, MOL-Molecular technique, NA—Not applicable.

Table 14. Prevalence and subtype distribution of Blastocystis spp. in reptiles in Asia (2010-2021).

\begin{tabular}{|c|c|c|c|c|c|c|}
\hline Host & Country & $\begin{array}{l}\text { No. of Samples } \\
\text { Examined }\end{array}$ & $\begin{array}{c}\text { Number of Positive } \\
\text { Samples (\%) }\end{array}$ & $\begin{array}{l}\text { Subtypes (STs) } \\
\text { Identified }\end{array}$ & Method(s) & References \\
\hline \multirow{4}{*}{$\begin{array}{c}\text { Squamata } \\
\text { Cobra snake } \\
\text { Albino python } \\
\text { Water monitor } \\
\text { lizard }\end{array}$} & & & & \multirow{4}{*}{$\begin{array}{c}\text { NA } \\
\text { NA } \\
\text { Unknown } \\
\text { (Clade VIII) }\end{array}$} & & \multirow{4}{*}{$\begin{array}{l}\text { Mirzapour et al. [370] } \\
\text { Mirzapour et al. [370] } \\
\text { Mohd Zain et al. [372] }\end{array}$} \\
\hline & Iran & 1 & $1(100.0)$ & & $\mathrm{CM}$ & \\
\hline & Iran & 1 & $1(100.0)$ & & $\mathrm{CM}$ & \\
\hline & Malaysia & 6 & $1(1.6)$ & & IVC, MOL & \\
\hline \multicolumn{7}{|l|}{ Testudines } \\
\hline African spurred & United Arab Emirates & 19 & $5(26.3)$ & Unknown & MOL & AbuOdeh et al. [369] \\
\hline Greek tortoise & United Arab Emirates & 2 & $1(50.0)$ & Unknown & MOL & AbuOdeh et al. [369] \\
\hline Iguana & United Arab Emirates & 1 & $1(100.0)$ & Unknown & MOL & AbuOdeh et al. [369] \\
\hline
\end{tabular}

CM-Conventional microscopy, IVC-In vitro cultivation, MOL-Molecular technique, NA-Not applicable.

Table 15. Prevalence and subtype distribution of Blastocystis spp. in insects and other animal groups in Asia (2010-2021).

\begin{tabular}{|c|c|c|c|c|c|c|}
\hline Host & Country & $\begin{array}{l}\text { No. of Samples } \\
\text { Examined }\end{array}$ & $\begin{array}{l}\text { Number of Positive } \\
\text { Samples (\%) }\end{array}$ & $\begin{array}{l}\text { Subtypes (STs) } \\
\text { Identified }\end{array}$ & Method(s) & References \\
\hline \multicolumn{7}{|l|}{ Blattodea } \\
\hline Cockroach & China & 116 & $96(82.8)$ & ST2 & MOL & Ma et al. [418] \\
\hline Cockroach & Thailand & 920 & $9(1.0)$ & NA & $\mathrm{CM}$ & Chamavit et al. [434] \\
\hline Cockroach & Thailand & 450 & $18(4.0)$ & NA & $\mathrm{CM}$ & $\begin{array}{l}\text { Dokmaikaw and } \\
\text { Suntaravitun [435] }\end{array}$ \\
\hline $\begin{array}{c}\text { Cockroach } \\
\text { (Blatella germanica) }\end{array}$ & Turkey & 138 & $57(41.0)$ & NA & $\mathrm{CM}$ & Oguz et al. [436] \\
\hline $\begin{array}{c}\text { Cockroach } \\
\text { (Blatella germanica) }\end{array}$ & Iran & 496 & $5(1.0)$ & NA & $\mathrm{CM}$ & $\begin{array}{c}\text { Motevalli-Haghi et al. } \\
\text { [437] }\end{array}$ \\
\hline $\begin{array}{c}\text { Cockroach } \\
\text { (Periplaneta americana) }\end{array}$ & Malaysia & 151 & $61(40.4)$ & ST3 & IVC, MOL & $\begin{array}{c}\text { Farah Haziqah et al. } \\
\text { [438] }\end{array}$ \\
\hline \multicolumn{7}{|l|}{ Diprotodontia } \\
\hline Gray kangaroo & China & 11 & $8(72.7)$ & ST10 & MOL & Zhao et al. [352] \\
\hline Red-necked wallaby & China & 15 & $2(13.3)$ & ST11 & MOL & Li et al. [353] \\
\hline Sugar glider & Indonesia & 100 & $100(100.0)$ & NA & CM, IVC & Natalia et al. [439] \\
\hline \multicolumn{7}{|l|}{ Lagomorpha } \\
\hline $\begin{array}{l}\text { New Zealand white } \\
\text { rabbit }\end{array}$ & China & 215 & $7(3.3)$ & ST4 & MOL & Wang et al. [373] \\
\hline Rabbit & China & 616 & $6(1.0)$ & NA & MOL & Li et al. [440] \\
\hline Rabbit & $\begin{array}{l}\text { United Arab } \\
\text { Emirates }\end{array}$ & 3 & $1(33.3)$ & ST14 & MOL & AbuOdeh et al. [369] \\
\hline \multicolumn{7}{|l|}{ Eulipotyphla } \\
\hline Hedgehog & Iran & 1 & $1(100.0)$ & NA & $\mathrm{CM}$ & Mirzapour et al. [370] \\
\hline
\end{tabular}


The prevalence of Blastocystis spp., reported in the last ten years, varied widely among the ungulates. Infection was mostly reported in livestock animals such as cattle, goats, sheep and pigs. Blastocystis spp. ST10 and ST14 were the most frequently isolated from deer, alpacas, cattle, yaks, sheep and goats, while ST1 and ST5 were the most common in pigs.

Blastocystis spp. has been isolated from carnivores, both domestic and wild, in Asia. Prevalence ranged from $0.6 \%$ to $100 \%$, with STs $1-8$ and ST10 being identified. NHPs have been commonly described to harbor Blastocystis spp., with a reported prevalence reaching a 100\%. Genetic analyses have recognized ST1, ST2, and ST3 as being the most common in this group of mammals. Interestingly, Blastocystis spp. ST9 was isolated from ring-tailed lemur from China [250].

Blastocystis spp. infections in birds have been reported. Prevalence varied widely, however, subtype identification revealed ST6, S7, ST8 as the most frequently isolated. The isolation of Blastocystis spp. ST9 in chicken in Malaysia [15] is peculiar. Diverse genera of rodents have been found as hosts to Blastocystis spp. Although STs 1, 3, 5, 7 and 13 have been reported, ST4 and ST17 were the most frequently identified.

A few studies have reported on the infection of reptiles with Blastocystis spp. with the highest sample size being 19. Prevalence ranged from $26.3 \%$ to $100 \%$, no subtype has yet been mentioned. Although studies are still few, cockroaches have been found as hosts to Blastocystis spp. Two out of six studies have described infection to the subtype level, ST2 was identified in China [418] while ST3 was identified in Malaysia [438].

Other animals found as hosts to Blastocystis spp. are the gray kangaroo, red-necked wallaby, sugar glider, rabbit, and hedgehog.

\section{Blastocystis spp. in Food and Environmental Sources}

In the past decade, the presence of Blastocystis spp. has been reported in tap water, river water, seawater, wells, fishponds, wastewater, food and even ambient air in Asia. The prevalence rate ranged from $2.1 \%$ to $100 \%$ in the various water sources, and $2.8 \%$ to $10.2 \%$ in leafy vegetables (Table 16). The only study on Blastocystis spp. in ambient air reported a prevalence of $1.4 \%$. Blastocystis spp. subtype identification is only available for water sources. STs 1, 2, 3, 4, 6, 8, 10 have so far been recorded from water samples; and although the prevalence of ST3 was highest, ST1 was the most widespread subtype.

Table 16. Prevalence and subtype distribution of Blastocystis spp. in food and environmental sources in Asia (2010-2021).

\begin{tabular}{|c|c|c|c|c|c|c|}
\hline Country & $\begin{array}{c}\text { Food/Environmental } \\
\text { Source }\end{array}$ & $\begin{array}{l}\text { No. of Samples } \\
\text { Examined }\end{array}$ & $\begin{array}{l}\text { No. of Positive } \\
\text { Samples (\%) }\end{array}$ & $\begin{array}{l}\text { Subtypes (STs) } \\
\text { Identified }\end{array}$ & Method(s) & References \\
\hline $\begin{array}{c}\text { Iran } \\
\text { Malaysia }\end{array}$ & $\begin{array}{l}\text { Treated wastewater } \\
\text { River water }\end{array}$ & $\begin{array}{c}12 \\
480\end{array}$ & $\begin{array}{c}5(41.7) \\
133(27.7)\end{array}$ & $\begin{array}{l}\text { ST2, ST6, ST8 } \\
\text { NA }\end{array}$ & $\begin{array}{l}\text { F, MOL } \\
\text { MB, IVC }\end{array}$ & $\begin{array}{l}\text { Javanmard et al. [441] } \\
\text { Ithoi et al. [442] }\end{array}$ \\
\hline Malaysia & $\begin{array}{l}\text { Drinking water } \\
\text { treatment plants }\end{array}$ & 85 & $22(25.9)$ & NA & IMS, CM & Richard et al. [443] \\
\hline Malaysia & $\begin{array}{l}\text { River water } \\
\text { Various water sources }\end{array}$ & 14 & $14(100.0)$ & $\begin{array}{l}\text { ST1, ST2, ST3 } \\
\text { ST1, ST2, ST3, } \\
\text { ST4, ST8, ST10 }\end{array}$ & MF, MOL & Noradilah et al. [444] \\
\hline Malaysia & $\begin{array}{c}\text { River water } \\
\text { Village water sources }\end{array}$ & $\begin{array}{c}7 \\
16\end{array}$ & $\begin{array}{c}3(42.9) \\
1(6.3)\end{array}$ & NA & MF, IVC & Noradilah et al. [23] \\
\hline Nepal & River water & 4 & $4(100.0)$ & ST1, ST4 & $\mathrm{C}, \mathrm{MOL}$ & Lee et al. [18] \\
\hline Philippines & $\begin{array}{l}\text { Wastewater (influent) } \\
\text { Wastewater (effluent) }\end{array}$ & $\begin{array}{l}31 \\
31\end{array}$ & $\begin{array}{l}7(23.0) \\
2(7.0)\end{array}$ & $\begin{array}{l}\text { ST1, ST2 } \\
\text { ST1, ST2 }\end{array}$ & C, IVC, MOL & Banaticla and Rivera, [445] \\
\hline Turkey & Tap water & 25 & $3(12.0)$ & ST1 & MOL & Eroglu and Koltas, [19] \\
\hline Turkey & $\begin{array}{l}\text { Streams and drinking } \\
\text { water }\end{array}$ & 228 & $47(20.6)$ & NA & $\mathrm{CM}$ & Karaman et al. [446] \\
\hline Turkey & $\begin{array}{l}\text { River water } \\
\text { Sea water }\end{array}$ & $\begin{array}{c}195 \\
48\end{array}$ & $\begin{array}{l}9(4.6) \\
1(2.1)\end{array}$ & $\begin{array}{l}\text { ST1, ST3 } \\
\text { ST1 }\end{array}$ & $\mathrm{C}, \mathrm{MOL}$ & Koloren et al. [447] \\
\hline $\begin{array}{l}\text { Turkey } \\
\text { Saudi Arabia } \\
\text { Iran }\end{array}$ & $\begin{array}{l}\text { Surface water } \\
\text { Leafy vegetables } \\
\text { Fresh vegetables }\end{array}$ & $\begin{array}{l}75 \\
470 \\
240\end{array}$ & $\begin{array}{l}4(5.3) \\
13(2.8) \\
10(4.2)\end{array}$ & $\begin{array}{l}\text { ST1, ST3 } \\
\text { NA } \\
\text { NA }\end{array}$ & $\begin{array}{l}\text { C, MOL } \\
\text { S, CM } \\
\text { S, CM }\end{array}$ & $\begin{array}{c}\text { Kolören and Karaman [448] } \\
\text { Al-Megrin [27] } \\
\text { Isazadeh et al. [449] }\end{array}$ \\
\hline Syria & Fresh vegetables & 128 & $13(10.2)$ & NA & MOL & $\begin{array}{c}\text { Al Nahhas and } \\
\text { Aboualchamat [450] }\end{array}$ \\
\hline Korea & Ambient air & 71 & $1(1.4)$ & NA & MOL & Han et al. [451] \\
\hline
\end{tabular}

C-Centrifugation, CM-Conventional microscopy, F-Filtration, IMS-Immunomagnetic separation technique, IVC-In vitro cultivation, MB-Membrane filtration, MOL-Molecular technique, S-Sedimentation, NA—Not applicable. 


\section{Distribution of Blastocystis spp. by Country}

From 2010 till now, the identification of Blastocystis spp. has been described for a total of 31 Asian countries. Out of these 31, genetic characterization and Blastocystis spp. subtype identification was available for 22 countries. Figure 1 reveals the distribution of the subtypes of Blastocystis spp. in these countries with a glimpse of subtypes shared by humans, animals, and water sources. Blastocystis spp. ST1 was the most widespread subtype, found in all of the 22 countries.

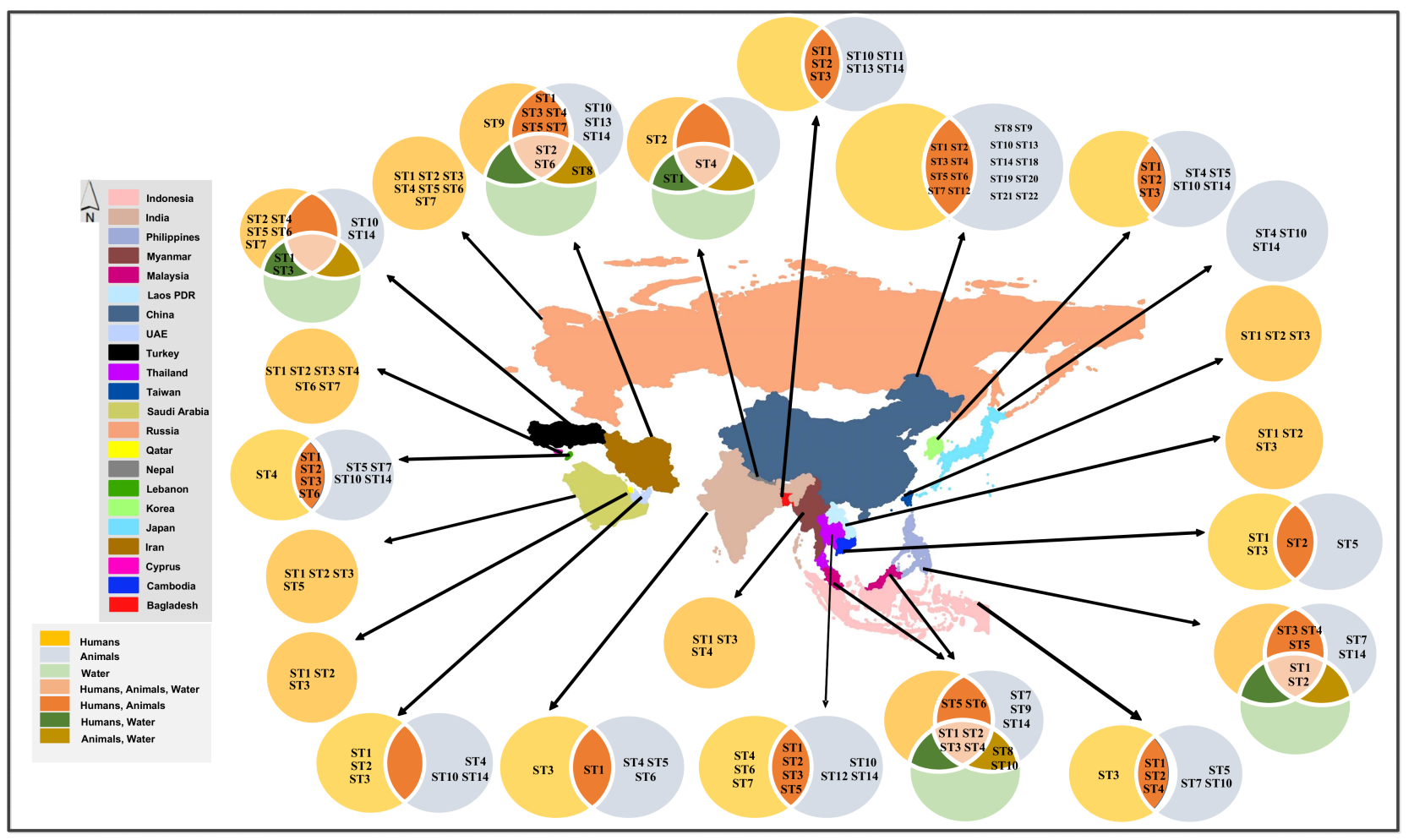

Figure 1. Distribution of Blastocystis spp. subtypes in Asia (2010-2021).

\section{Discussion}

Blastocystis spp. have been reported in over $50 \%$ of the countries in the continent of Asia. Although the most documented hosts to infection were humans and several animal species, this organism has also been detected in water sources, vegetables, and ambient air.

Variation of prevalence rates was seen within and between the various human host categories. Although authors have described both significant and insignificant differences between Blastocystis spp. infection in patients with and without known disease conditions, this variation could be a result of the methods employed in the detection of Blastocystis spp. Blastocystis spp. STs 1-7 have been identified in humans in Asia. ST1, ST2, ST3 and ST4 were more widespread and more frequently isolated than ST5, ST6 and ST7. This finding is in agreement with studies from other parts of the world [2,13,452,453].

The isolation of Blastocystis spp. STs 1-14, and ST18-22 (novel subtypes) were reported in animal hosts. ST1, ST2, ST3, ST4, ST5, ST6 and ST7 were found common to humans and animals. ST9 was observed in ring-tailed lemurs and chickens in China [250] and Malaysia [15] respectively; however, no article included in this review reported on the identification of ST9 in humans in these countries. The characteristic presence of ST5 in pigs, ST10 and ST14 in goats, sheep and cattle, and ST6 and ST7 in chickens underscore suggestions that these STs are specific to the respective animal hosts. Also, reports of isolation of ST5 in pig handlers [214] and ST6 in chicken slaughterhouse staff [199] are pointers to possible zoonotic transmission. 
Where stated, cysts were the Blastocystis spp. forms observed in vegetables and water samples. The presence of cysts in the life cycle of Blastocystis spp. enable their existence outside of human and animal hosts; also, the chloroform-resistant nature of these cysts probably explains the presence of Blastocystis spp. even in treated water.

\section{Conclusions}

The growing interest in the study of Blastocystis spp. as an area of research is very obvious and fundamental to unraveling the much that is hitherto unknown of the epidemiology, biology and pathogenicity of this protist. Blastocystis spp. have been isolated from biotic and abiotic sources in Asia. Considering that humans and animals are in constant interactions with their environment, epidemiological studies of Blastocystis spp. from an ecological perspective are essential. In essence, continuous surveillance of human and animal hosts alongside their food and water sources and other possible sources of infection such as soil across different geographical locations and climatic conditions is needed. The use of molecular detection methods in epidemiological studies are recommended to provide information on Blastocystis spp. STs in as many regions as possible. Incorporating the One Health $(\mathrm{OH})$ method into epidemiological studies will equip researchers and other stakeholders with information on the possible influence of ecosystems on Blastocystis spp., it will further elucidate transmission routes and provide clues required to break the transmission of this protist successfully. Morphological studies of Blastocystis spp. in various host species and environmental sources are insufficient but essential; electron microscopy could help to accentuate structural details of isolates from various hosts and the differences or similarities between them, and contribute to the understanding of a proper, more detailed Blastocystis spp. lifecycle.

Author Contributions: Conceptualization, I.L.L., F.H.M.T. and N.S.; writing-original draft preparation, A.A.R.-A.; writing-review and editing, I.L.L., F.H.M.T. and N.S.; All authors have read and agreed to the published version of the manuscript.

Funding: This work was supported by Short Term Grants of UniSHAMS (100-161(RMU)01/STG/2020 Bil. (62)).

Institutional Review Board Statement: Not applicable.

Informed Consent Statement: Not applicable.

Data Availability Statement: All data generated or analyzed during this study are included in this published article.

Conflicts of Interest: The authors declare no conflict of interest.

\section{References}

1. Tan, K.S.W. New Insights on Classification, Identification, and Clinical Relevance of Blastocystis spp. Clin. Microbiol. Rev. 2008, 21, 639-665. [CrossRef] [PubMed]

2. Alfellani, M.A.; Stensvold, C.R.; Vidal-Lapiedra, A.; Onuoha, E.S.U.; Fagbenro-Beyioku, A.F.; Clark, C.G. Variable Geographic Distribution of Blastocystis Subtypes and Its Potential Implications. Acta Trop. 2013, 126, 11-18. [CrossRef] [PubMed]

3. Deng, L.; Yao, J.; Chen, S.; He, T.; Chai, Y.; Zhou, Z.; Shi, X.; Liu, H.; Zhong, Z.; Fu, H.; et al. First Identification and Molecular Subtyping of Blastocystis sp. in Zoo Animals in Southwestern China. Parasites Vectors 2021, 14, 11. [CrossRef]

4. Andersen, L.O.; Stensvold, C.R. Blastocystis in Health and Disease: Are We Moving from a Clinical to a Public Health Perspective? J. Clin. Microbiol. 2016, 54, 524-528. [CrossRef] [PubMed]

5. Stenzel, D.J.; Boreham, P.F.L. Blastocystis hominis Revisited. Clin. Microbiol. Rev. 1996, 105, 563-584. [CrossRef]

6. Silberman, J.D.; Sogin, M.L.; Leipe, D.D.; Clark, C.G. Human Parasite Finds Taxonomic Home. Nature 1996, 380, 398. [CrossRef]

7. Yoshikawa, H.; Koyama, Y.; Tsuchiya, E.; Takami, K. Blastocystis Phylogeny among Various Isolates from Humans to Insects. Parasitol. Int. 2016, 65, 750-759. [CrossRef]

8. Lepczyńska, M.; Białkowska, J.; Dzika, E.; Piskorz-Ogórek, K.; Korycińska, J. Blastocystis: How Do Specific Diets and Human Gut Microbiota Affect Its Development and Pathogenicity? Eur. J. Clin. Microbiol. Infect. Dis. 2017, 36, 1531-1540. [CrossRef]

9. Parija, S.; Jeremiah, S. Blastocystis: Taxonomy, Biology and Virulence. Trop. Parasitol. 2013, 3, 17. [CrossRef]

10. Stensvold, C.R.; Suresh, G.K.; Tan, K.S.W.; Thompson, R.C.A.; Traub, R.J.; Viscogliosi, E.; Yoshikawa, H.; Clark, C.G. Terminology for Blastocystis Subtypes-a Consensus. Trends Parasitol. 2007, 23, 93-96. [CrossRef] 
11. Stensvold, C.R.; Clark, C.G. Pre-Empting Pandora's Box: Blastocystis Subtypes Revisited. Trends Parasitol. 2020, 36, $229-232$. [CrossRef] [PubMed]

12. Hublin, J.S.Y.; Maloney, J.G.; Santin, M. Blastocystis in Domesticated and Wild Mammals and Birds. Res. Vet. Sci. 2020, 135, 260-282. [CrossRef] [PubMed]

13. Ramírez, J.D.; Sánchez, A.; Hernández, C.; Flórez, C.; Bernal, M.C.; Giraldo, J.C.; Reyes, P.; López, M.C.; García, L.; Cooper, P.J. Geographic Distribution of Human Blastocystis Subtypes in South America. Infect. Genet. Evol. 2016, 41, 32-35. [CrossRef] [PubMed]

14. Zhang, Q.; Yin, W.; Wang, X.; Zhang, Z.; Zhang, R.; Duan, Z. Blastocystis Infection and Subtype Distribution in Domestic Animals in the Qinghai-Tibetan Plateau Area (QTPA) in China: A Preliminary Study. Parasitol. Int. 2021, 81, 102272. [CrossRef] [PubMed]

15. Noradilah, S.A.; Anuar, T.S.; Moktar, N.; Lee, I.L.; Salleh, F.M.; Azreen, S.N.A.M.; Husnie, N.S.M.M.; Azrul, S.M.; Abdullah, W.O.; Nordin, A.; et al. Molecular Epidemiology of Blastocystis sp. in Animals Reared by the Aborigines during Wet and Dry Seasons in Rural Communities, Pahang, Malaysia. Southeast Asian J. Trop. Med. Public Health 2017, 48, 1151-1160.

16. Yoshikawa, H.; Yoshida, K.; Nakajima, A.; Yamanari, K.; Iwatani, S.; Kimata, I. Fecal-Oral Transmission of the Cyst Form of Blastocystis hominis in Rats. Parasitol. Res. 2004, 94, 391-396. [CrossRef]

17. Anuar, T.S.; Ghani, M.K.A.; Azreen, S.N.; Salleh, F.M.; Moktar, N. Blastocystis Infection in Malaysia: Evidence of Waterborne and Human-to-Human Transmissions among the Proto-Malay, Negrito and Senoi Tribes of Orang Asli. Parasites Vectors 2013, 6, 1. [CrossRef]

18. Lee, L.; Chye, T.; Karmacharya, M.; Govind, S. Blastocystis sp.: Waterborne Zoonotic Organism, a Possibility? Parasites Vectors 2012, 5, 130. [CrossRef]

19. Rajah Salim, H.; Kumar, G.S.; Vellayan, S.; Mak, J.W.; Khairul Anuar, A.; Init, I.; Vennila, G.D.; Saminathan, R.; Ramakrishnan, K. Blastocystis in Animal Handlers. Parasitol. Res. 1999, 85, 1032-1033. [CrossRef]

20. Rivera, W.L. Phylogenetic Analysis of Blastocystis Isolates from Animal and Human Hosts in the Philippines. Vet. Parasitol. 2008, 156, 178-182. [CrossRef]

21. Parkar, U.; Traub, R.J.; Vitali, S.; Elliot, A.; Levecke, B.; Robertson, I.; Geurden, T.; Steele, J.; Drake, B.; Thompson, R.C.A. Molecular Characterization of Blastocystis Isolates from Zoo Animals and Their Animal-Keepers. Vet. Parasitol. 2010, 169, 8-17. [CrossRef]

22. Noradilah, S.A.; Lee, I.L.; Anuar, T.S.; Salleh, F.M.; Abdul Manap, S.N.A.; Husnie, N.S.; Azrul, S.M.; Moktar, N. Blastocystis spp. Contaminated Water Sources in Aboriginal Settlements. Trop. Biomed. 2017, 34, 110-117. [PubMed]

23. Leelayoova, S.; Siripattanapipong, S.; Thathaisong, U.; Naaglor, T.; Taamasri, P.; Piyaraj, P.; Mungthin, M. Drinking Water: A Possible Source of Blastocystis spp. Subtype 1 Infection in Schoolchildren of a Rural Community in Central Thailand. Am. J. Trop. Med. Hyg. 2008, 79, 401-406. [CrossRef] [PubMed]

24. Rodrigues, A.C.; da Silva, M.D.C.; Pereira, R.Â.S.; Pinto, L.C. Prevalence of Contamination by Intestinal Parasites in Vegetables (Lactuca sativa L. and Coriandrum sativum L.) Sold in Markets in Belém, Northern Brazil. J. Sci. Food Agric. 2020, 100, $2859-2865$. [CrossRef] [PubMed]

25. Caradonna, T.; Marangi, M.; Del Chierico, F.; Ferrari, N.; Reddel, S.; Bracaglia, G.; Normanno, G.; Putignani, L.; Giangaspero, A. Detection and Prevalence of Protozoan Parasites in Ready-to-Eat Packaged Salads on Sale in Italy. Food Microbiol. 2017, 67, 67-75. [CrossRef]

26. Al-Megrin, W.A.I. Prevalence Intestinal Parasites in Leafy Vegetables in Riyadh, Saudi Arabia. Int. J. Trop. Med. 2010, 5, 20-23. [CrossRef]

27. Eroglu, F.; Koltas, I.S. Evaluation of the Transmission Mode of B. hominis by Using PCR Method. Parasitol. Res. 2010, 107, 841-845. [CrossRef]

28. World Health Organization. Microbial Fact Sheets. In World Health Organization Guidelines for Drinking-Water Quality (WHO GDWQ); Gutenberg: Triq Tal Barrani, Malta, 2011; pp. 231-305.

29. Shrestha, K.; Acharya, K.P.; Shrestha, S. One Health: The Interface between Veterinary and Human Health. Int. J. One Health 2018, 4, 8-14. [CrossRef]

30. Mackenzie, J.S.; Jeggo, M. The One Health Approach-Why Is It So Important? Trop. Med. Infect. Dis. 2019, 4, 88. [CrossRef]

31. Mackenzie, J.; McKinnon, M.; Jeggo, M. One Health: From Concept to Practice. In Confronting Emerging Zoonoses: The One Health Paradigm; Yamada, A., Kahn, L.H., Kaplan, B., Monath, T.P., Woodall, J., Conti, L., Eds.; Springer: Tokyo, Japan, 2014; pp. 163-189. [CrossRef]

32. Adao, D.E.V.; Rivera, W.L. Recent Advances in Blastocystis sp. Research. Philipp. Sci. Lett. 2018, 11, 39-60. [CrossRef]

33. Rauff-Adedotun, A.A.; Mohd Zain, S.N.; Farah Haziqah, M.T. Current Status of Blastocystis sp. in Animals from Southeast Asia: A Review. Parasitol. Res. 2020, 119, 3559-3570. [CrossRef] [PubMed]

34. Barua, P.; Khanum, H.; Haque, R.; Najib, F.; Kabir, M. Establishment of Blastocystis hominis In-Vitro Culture Using Fecal Samples from Infants in Slum Area of Mirpur, Dhaka, Bangladesh. Acta Med. Int. 2015, 2, 40. [CrossRef]

35. Ben-Shimol, S.; Sagi, O.; Greenberg, D. Differences in Prevalence of Parasites in Stool Samples between Three Distinct Ethnic Pediatric Populations in Southern Israel, 2007-2011. Parasitol. Int. 2014, 63, 456-462. [CrossRef] [PubMed]

36. Zhang, S.-X.; Zhou, Y.-M.; Xu, W.; Tian, L.-G.; Chen, J.-X.; Chen, S.-H.; Dang, Z.-S.; Gu, W.-P.; Yin, J.-W.; Serrano, E.; et al. Impact of Co-Infections with Enteric Pathogens on Children Suffering from Acute Diarrhea in Southwest China. Infect. Dis. Poverty 2016, 5, 64. [CrossRef] [PubMed]

37. Qi, M.; Wei, Z.; Zhang, Y.; Zhang, Q.; Li, J.; Zhang, L.; Wang, R. Genetic Diversity of Blastocystis in Kindergarten Children in Southern Xinjiang, China. Parasites Vectors 2020, 13, 1-6. [CrossRef] [PubMed]

38. Ning, C.-Q.; Kang, J.-M.; Li, Y.-T.; Chen, H.-H.; Chu, Y.-H.; Yu, Y.-F.; Wu, X.-P.; Ai, L.; Chen, J.-X.; Tian, L.-G.; et al. Prevalence and Risk Factors of Blastocystis Infections among Primary School Students in Jiangjin District, Chongqing City. Chin. J. Schistosomiasis Control. 2020, 32, 489-497. [CrossRef] 
39. Liao, C.-W.; Chiu, K.-C.; Chiang, I.-C.; Cheng, P.-C.; Chuang, T.-W.; Kuo, J.-H.; Tu, Y.-H.; Fan, C.-K. Prevalence and Risk Factors for Intestinal Parasitic Infection in Schoolchildren in Battambang, Cambodia. Am. J. Trop. Med. Hyg. 2017, 96, 583-588. [CrossRef]

40. Rayan, P.; Verghese, S.; McDonnell, P.A. Geographical Location and Age Affects the Incidence of Parasitic Infestations in School Children. Indian J. Pathol. Microbiol. 2010, 53, 504-508. [CrossRef]

41. Yoshikawa, H.; Tokoro, M.; Nagamoto, T.; Arayama, S.; Asih, P.B.S.; Rozi, I.E.; Syafruddin, D. Molecular Survey of Blastocystis sp. from Humans and Associated Animals in an Indonesian Community with Poor Hygiene. Parasitol. Int. 2016, 65, 780-784. [CrossRef]

42. Zulfa, F.; Sari, I.P.; Kurniawan, A. Association of Blastocystis Subtypes with Diarrhea in Children. J. Phys. Conf. Ser. 2017, 884, 012031. [CrossRef]

43. Sari, I.P.; Benung, M.R.; Wahdini, S.; Kurniawan, A. Diagnosis and Identification of Blastocystis Subtypes in Primary School Children in Jakarta. J. Trop. Pediatr. 2018, 64, 208-214. [CrossRef]

44. Subahar, R.; Susanto, L.; Astuty, H.; Winita, R.; Sari, I.P. Intestinal Parasitic Infections and Hemoglobin Levels among Schoolchildren Participating in a Deworming Program in Jakarta, Indonesia: A Cross-Sectional Study. Open Access Maced. J. Med. Sci. 2020, 8, 589-594. [CrossRef]

45. Sari, I.P.; Audindra, S.; Zhafira, A.S.; Rahma, A.A.; Syarira, C.V.; Wahdini, S. Nutritional Status of School-Aged Children with Intestinal Parasite Infection in South Jakarta, Indonesia. Open Access Maced. J. Med. Sci. 2021, 9, 95-100. [CrossRef]

46. Ashtiani, M.T.H.; Monajemzadeh, M.; Saghi, B.; Shams, S.; Mortazavi, S.H.; Khaki, S.; Mohseni, N.; Kashi, L.; Nikmanesh, B. Prevalence of Intestinal Parasites among Children Referred to Children's Medical Center during 18 Years (1991-2008), Tehran, Iran. Ann. Trop. Med. Parasitol. 2011, 105, 507-512. [CrossRef] [PubMed]

47. Niaraki, S.R.; Hajialilo, E.; Delshad, A.; Alizadeh, S.A.; Alipour, M.; Heydarian, P.; Saraei, M. Molecular Epidemiology of Blastocystis spp. in Children Referred to Qods Hospital in Northwest of Iran. J. Parasit. Dis. 2020, 44, 151-158. [CrossRef] [PubMed]

48. Mahmoudvand, H.; Taee, N.; Faraji Goodarzi, M.; Ebrahimzadeh, F. Prevalence and Risk Factors of Intestinal Protozoan Infections in Children (2-15 Yr Old) from Lorestan Province, Western Iran. Trop. Biomed. 2018, 35, 259-266.

49. Abdi, J.; Farhadi, M.; Aghaee, S. Prevalence of Intestinal Parasites among Children Attending the Daycare Centers of Ilam, Western Iran. J. Med. Sci. 2014, 14, 143-146. [CrossRef]

50. Daryani, A.; Sharif, M.; Nasrolahei, M.; Khalilian, A.; Mohammadi, A.; Barzegar, G. Epidemiological Survey of the Prevalence of Intestinal Parasites among Schoolchildren in Sari, Northern Iran. Trans. R. Soc. Trop. Med. Hyg. 2012, 106, 455-459. [CrossRef] [PubMed]

51. Hazrati Tappeh, K.H.; Mostaghim, M.; Hanifian, H.; Khalkhali, H.; Mousavi, J. A Study on the Intestinal Parasitic Infections among Elementary School Students at a District (Silvana) In Urmia, West Azerbaijan. Int. J. Res. Appl. Basic Med. Sci. 2015, 1, 14-19. [CrossRef]

52. Norouzi, R.; Nourian, A.; Hanilo, A.; Kamali, K. Prevalence of Intestinal Parasites among Primary School Students in Zanjan City (2013). J. Zanjan Univ. Med. Sci. Health Serv. 2016, 24, 121-130.

53. Babakhani, M.; Safari, R.; Rajati, F.; Salimi, S.; Omidian doost, A. Prevalence and Risk Factors Associated with Intestinal Parasitic Infections among School Children in Gashky, West of Iran. Int. J. Pediatr. 2017, 5, 5263-5273. [CrossRef]

54. Bahmani, P.; Maleki, A.; Sadeghi, S.; Shahmoradi, B.; Ghahremani, E. Prevalence of Intestinal Protozoa Infections and Associated Risk Factors among Schoolchildren in Sanandaj City, Iran. Iran. J. Parasitol. 2017, 12, 108-116.

55. Saki, J.; Amraee, D. Prevalence of Intestinal Parasites Among the Rural Primary School Students in the West of Ahvaz County, Iran, 2015. Jentashapir J. Health Res. 2017, 8, e40326. [CrossRef]

56. Turki, H.; Hamedi, Y.; Heidari-Hengami, M.; Najafi-Asl, M.; Rafati, S.; Sharifi-Sarasiabi, K. Prevalence of Intestinal Parasitic Infection among Primary School Children in Southern Iran. J. Parasit. Dis. 2017, 41, 659-665. [CrossRef] [PubMed]

57. Mahdi, N.K.; Al-Saadoon, M.A. Microsporidiosis among Children with Malignant Diseases in Basrah, Iraq. Pak. J. Med. Sci. 2012, $28,621-624$

58. Osman, M.; El Safadi, D.; Cian, A.; Benamrouz, S.; Nourrisson, C.; Poirier, P.; Pereira, B.; Razakandrainibe, R.; Pinon, A.; Lambert, C.; et al. Prevalence and Risk Factors for Intestinal Protozoan Infections with Cryptosporidium, Giardia, Blastocystis and Dientamoeba among Schoolchildren in Tripoli, Lebanon. PLoS Negl. Trop. Dis. 2016, 10, 1-17. [CrossRef]

59. Abd. Ghani, M.K.; Yusof, H. Blastocystis hominis: Kehadirannya Di Dalam Sampel Feses Kanak-Kanak Orang Asli Di Pos Lenjang, Pahang, Malaysia. Sains Malays. 2011, 40, 1123-1127.

60. Abdulsalam, A.M.; Ithoi, I.; Al-Mekhlafi, H.M.; Ahmed, A.; Surin, J.; Mak, J.-W. Drinking Water Is a Significant Predictor of Blastocystis Infection among Rural Malaysian Primary Schoolchildren. Parasitology 2012, 139, 1014-1020. [CrossRef]

61. Al-Harazi, T.; Ghani, M.K.A.; Othman, H. Prevalence of Intestinal Protozoan Infections among Orang Asli Schoolchildren in Pos Senderut, Pahang, Malaysia. J. Egypt. Soc. Parasitol. 2013, 43, 561-568. [CrossRef]

62. Sinniah, B.; Hassan, A.K.R.; Sabaridah, I.; Soe, M.M.; Ibrahim, Z.; Ali, O. Prevalence of Intestinal Parasitic Infections among Communities Living in Different Habitats and Its Comparison with One Hundred and One Studies Conducted over the Past 42 Years (1970 to 2013) in Malaysia. Trop. Biomed. 2014, 31, 190-206.

63. Nithyamathi, K.; Chandramathi, S.; Kumar, S. Predominance of Blastocystis sp. Infection among School Children in Peninsular Malaysia. PLoS ONE 2016, 11, e0136709. [CrossRef]

64. Tang, S.G.H.; Kamel, A.G.M. Intestinal Protozoan Infections of Schoolchildren in an Aboriginal (Orang Asli) Settlement in Perak, Malaysia. Int. Med. J. 2020, 27, 31-35.

65. Adli, M.N.; Mohamed Kamel, A.G. Blastocystosis amongst the Orang Asli (Aborigine) School Children of Sktar Kuala Kubu Bharu, Selangor, Malaysia. Int. Med. J. 2020, 27, 412-414. 
66. Mukhiya, R.K.; Rai, S.K.; Karki, A.B.; Prajapati, A. Intestinal Protozoan Parasitic Infection among School Children. J. Nepal Health Res. Counc. 2012, 10, 204-207. [PubMed]

67. Al-Mohammed, H.I.; Amin, T.T.; Aboulmagd, E.; Hablus, H.R.; Zaza, B.O. Prevalence of Intestinal Parasitic Infections and Its Relationship with Socio-Demographics and Hygienic Habits among Male Primary Schoolchildren in Al-Ahsa, Saudi Arabia. Asian Pac. J. Trop. Med. 2010, 3, 906-912. [CrossRef]

68. Bakarman, M.A.; Hegazi, M.A.; Butt, N.S. Prevalence, Characteristics, Risk Factors, and Impact of Intestinal Parasitic Infections on School Children in Jeddah, Western Saudi Arabia. J. Epidemiol. Glob. Health 2019, 9, 81-87. [CrossRef] [PubMed]

69. Suntaravitun, P.; Dokmaikaw, A. Prevalence of Intestinal Protozoan Infections among Schoolchildren in Bang Khla District, Chachoengsao Province, Central Thailand. Asian Pac. J. Trop. Dis. 2017, 7, 523-526. [CrossRef]

70. Sanprasert, V.; Srichaipon, N.; Bunkasem, U.; Srirungruang, S.; Nuchprayoon, S. Prevalence of Intestinal Protozoan Infections among Children in Thailand: A Large-Scale Screening and Comparative Study of Three Standard Detection Methods. Southeast Asian J. Trop. Med. Public Health 2016, 47, 1123-1133. [PubMed]

71. Thathaisong, U.; Siripattanapipong, S.; Mungthin, M.; Pipatsatitpong, D.; Tan-Ariya, P.; Naaglor, T.; Leelayoova, S. Identification of Blastocystis Subtype 1 Variants in the Home for Girls, Bangkok, Thailand. Am. J. Trop. Med. Hyg. 2013, 88, 352-358. [CrossRef] [PubMed]

72. Pipatsatitpong, D.; Leelayoova, S.; Mungthin, M.; Aunpad, R.; Naaglor, T.; Rangsin, R. Prevalence and Risk Factors for Blastocystis Infection among Children and Caregivers in a Child Care Center, Bangkok, Thailand. Am. J. Trop. Med. Hyg. 2015, 93, 310-315. [CrossRef] [PubMed]

73. Punsawad, C.; Phasuk, N.; Bunratsami, S.; Thongtup, K.; Viriyavejakul, P.; Palipoch, S.; Koomhin, P.; Nongnaul, S. Prevalence of Intestinal Parasitic Infections and Associated Risk Factors for Hookworm Infections among Primary Schoolchildren in Rural Areas of Nakhon Si Thammarat, Southern Thailand 11 Medical and Health Sciences 1117 Public Health and Health Services. BMC Public Health 2018, 18, 1118. [CrossRef]

74. Assavapongpaiboon, B.; Bunkasem, U.; Sanprasert, V.; Nuchprayoon, S. A Cross-Sectional Study on Intestinal Parasitic Infections in Children in Suburban Public Primary Schools, Saraburi, the Central Region of Thailand. Am. J. Trop. Med. Hyg. 2018, 98, 763-767. [CrossRef]

75. Boondit, J.; Pipatsatitpong, D.; Mungthin, M.; Taamasri, P.; Tan-Ariya, P.; Naaglor, T.; Leelayoova, S. Incidence and Risk Factors of Blastocystis Infection in Orphans at the Babies' Home, Nonthaburi Province, Thailand. J. Med. Assoc. Thail. 2014, 97, S52-S59.

76. Kitvatanachai, S.; Rhongbutsri, P. Intestinal Parasitic Infections in Suburban Government Schools, Lak Hok Subdistrict, Muang Pathum Thani, Thailand. Asian Pac. J. Trop. Med. 2013, 6, 699-702. [CrossRef]

77. Popruk, S.; Thima, K.; Udonsom, R.; Rattaprasert, P.; Sukthana, Y. Does Silent Giardia Infection Need Any Attention? Open Trop. Med. J. 2011, 4, 26-32. [CrossRef]

78. Güdücüoğlu, H.; Parlak, M.; Ciçek, M.; Yaman, G.; Oztürk, O.; Cikman, A.; Berktaş, M. [Investigation of Intestinal Parasites in Students of Mustafa Cengiz Primary School in Van]. I Van Mustafa Cengiz Ilköğretim Okulu Öğrencilerinde Bağirsak Parazitlerinin Araştirilmasi. Turk. Parazitolojii Derg. 2010, 34, 172-175.

79. Hamamci, B.; Cetinkaya, U.; Delice, S.; Erçal, B.D.; Gücüyetmez, S.; Yazar, S. [Investigation of Intestinal Parasites among Primary School Students in Kayseri-Hacilar]. Kayseri-Hacilar'da İlköğretim Okulu Öğrencilerinde Bağirsak Parazitlerinin Araştirilmasi. Turk. Parazitolojii Derg. 2011, 35, 96-99. [CrossRef]

80. Sankur, F.; Ayturan, S.; Malatyali, E.; Ertabaklar, H.; Ertug, S. The Distribution of Blastocystis Subtypes among School-Aged Children in Mugla, Turkey. Iran. J. Parasitol. 2017, 12, 580-586. [PubMed]

81. Calik, S.; Karaman, U.; Colak, C. Prevalence of Microsporidium and Other Intestinal Parasites in Children from Malatya, Turkey. Indian J. Microbiol. 2011, 51, 345-349. [CrossRef] [PubMed]

82. Dogan, N.; Aydin, M.; Tuzemen, N.U.; Dinleyici, E.C.; Oguz, I.; Dogruman-Al, F. Subtype Distribution of Blastocystis spp. Isolated from Children in Eskisehir, Turkey. Parasitol. Int. 2017, 66, 948-951. [CrossRef] [PubMed]

83. Salehi Kahish, R.; Alghasi, A.; Hadadi, S.; Nasab, M.A.; Mafakherzadeh, A. The Prevalence of Blastocystis Infection in Pediatric Patients with Malignancy: A Single-Center Study in Ahvaz, Iran. Arch. Pediatr. Infect. Dis. 2021, 9, e104068. [CrossRef]

84. Asghari, A.; Zare, M.; Hatam, G.; Shahabi, S.; Gholizadeh, F.; Motazedian, M. Molecular Identification and Subtypes Distribution of Blastocystis sp. Isolated from Children and Adolescent with Cancer in Iran: Evaluation of Possible Risk Factors and Clinical Features. Acta Parasitol. 2020, 65, 462-473. [CrossRef] [PubMed]

85. Salehi Kahyesh, R.; Alghasi, A.; Haddadi, S.; Sharhani, A. Intestinal Parasites Infection in Children with Cancer in Ahvaz, Southwest Iran. Interdiscip. Perspect. Infect. Dis. 2020, 2020. [CrossRef] [PubMed]

86. Zabolinejad, N.; Berenji, F.; Eshkaftaki, E.B.; Badeii, Z.; Banihashem, A.; Afzalaqaei, M. Intestinal Parasites in Children with Lymphohematopoietic Malignancy in Iran, Mashhad. Jundishapur J. Microbiol. 2013, 6, e7765. [CrossRef]

87. Mahmoudvand, H.; Sepahvand, A.; Badparva, E.; Khatami, M.; Niazi, M.; Moayyedkazemi, A. Possible Association and Risk Factors of Blastocystis Infection and Colorectal Cancers in Western Iran. Arch. Clin. Infect. Dis. 2021, 16, e90861. [CrossRef]

88. Zhang, W.; Ren, G.; Zhao, W.; Yang, Z.; Shen, Y.; Sun, Y.; Liu, A.; Cao, J. Genotyping of Enterocytozoon Bieneusi and Subtyping of Blastocystis in Cancer Patients: Relationship to Diarrhea and Assessment of Zoonotic Transmission. Front. Microbiol. 2017, 8, 1835. [CrossRef] [PubMed]

89. Chandramathi, S.; Suresh, K.; Anita, Z.B.; Kuppusamy, U.R. Infections of Blastocystis hominis and Microsporidia in Cancer Patients: Are They Opportunistic? Trans. R. Soc. Trop. Med. Hyg. 2012, 106, 267-269. [CrossRef]

90. Mohamed, A.M.; Ahmed, M.A.; Ahmed, S.A.; Al-Semany, S.A.; Alghamdi, S.S.; Zaglool, D.A. Predominance and Association Risk of Blastocystis hominis Subtype i in Colorectal Cancer: A Case Control Study. Infect. Agent. Cancer 2017, 12, 1-8. [CrossRef] 
91. Yersal, O.; Malatyali, E.; Ertabaklar, H.; Oktay, E.; Barutca, S.; Ertug, S. Blastocystis Subtypes in Cancer Patients: Analysis of Possible Risk Factors and Clinical Characteristics. Parasitol. Int. 2016, 65, 792-796. [CrossRef]

92. Mülayim, S.; Aykur, M.; Dağcı, H.; Dalkılıç, S.; Aksoy, A.; Kaplan, M. Investigation of Isolated Blastocystis Subtypes from Cancer Patients in Turkey. Acta Parasitol. 2021, 66, 584-592. [CrossRef]

93. Teng, X.J.; Chu, Y.H.; Zhai, C.C.; Yu, Y.F.; Cai, Y.C.; Chen, S.H.; Ai, L.; Tian, L.G.; Chen, J.X. The Epidemiological Characteristics and Infuencing Factors for Blastocystis hominis Infection among Human Immunodefciency Virus Seropositive Individuals in Tengchong of Yunnan Province. Zhongguo Ji Sheng Chong Xue Yu Ji Sheng Chong Bing Za Zhi 2018, 36, 129-134.

94. Tian, L.-G.; Wang, T.-P.; Chen, J.-X.; Cai, Y.-C.; Yin, X.-M.; Cheng, G.-J.; Wu, W.-D.; Steinmann, P.; Guo, J.; Tong, X.-M.; et al. Co-Infection of HIV and Parasites in China: Results from an Epidemiological Survey in Rural Areas of Fuyang City, Anhui Province, China. Front. Med. China 2010, 4, 192-198. [CrossRef]

95. Tian, L.-G.; Chen, J.-X.; Wang, T.-P.; Cheng, G.-J.; Steinmann, P.; Wang, F.-F.; Cai, Y.-C.; Yin, X.-M.; Guo, J.; Zhou, L.; et al. Co-Infection of HIV and Intestinal Parasites in Rural Area of China. Parasites Vectors 2012, 5, 36. [CrossRef] [PubMed]

96. Tian, L.-G.; Wang, T.-P.; Lv, S.; Wang, F.-F.; Guo, J.; Yin, X.-M.; Cai, Y.-C.; Dickey, M.K.; Steinmann, P.; Chen, J.-X. HIV and Intestinal Parasite Co-Infections among a Chinese Population: An Immunological Profile. Infect. Dis. Poverty 2013, 2, 18. [CrossRef]

97. Zhang, S.-X.; Yu, Y.-F.; Wu, X.-P.; Chu, Y.-H.; Teng, X.-J.; Wang, F.-F.; Chen, J.-X.; Tian, L.-G. Epidemiological Characteristics and Risk Factors of Blastocystis hominis Infection among Patients with Hiv/Aids in Fuyang City, Anhui Province. Chin. J. Schistosomiasis Control 2019, 31, 498-503. [CrossRef]

98. Zhang, S.-X.; Kang, F.-Y.; Chen, J.-X.; Tian, L.-G.; Geng, L.-L. Risk Factors for Blastocystis Infection in HIV / AIDS Patients with Highly Active Antiretroviral Therapy in Southwest China. Infect. Dis. Poverty 2019, 8, 89. [CrossRef] [PubMed]

99. Zhu-Hua, H.; Hui-Hui, C.; Ke, Q.; Chao-Qun, N.; Guo-Hua, P.; Ying-Fang, Y.; Xian-Feng, Z.; Yan-Hong, C.; Dan, X.; Jia-Xu, C.; et al. Prevalence and Risk Factors of Blastocystis hominis Infections among AIDS Patients in Nanchang City. Chin. J. Schistosomiasis Control 2020, 32, 557-583. [CrossRef]

100. Ramana, K.V.; Prakash, K.; Mohanty, S.K. A Study of Opportunistic Parasitic Infections and CD4 Counts in HIV-Seropositive Individuals in Narketpally, South India. Ann. Trop. Med. Public Health 2010, 3, 49-52. [CrossRef]

101. Khalil, S.; Mirdha, B.R.; Sinha, S.; Panda, A.; Singh, Y.; Joseph, A.; Deb, M. Intestinal Parasitosis in Relation to Anti-Retroviral Therapy, CD4 ${ }^{+}$T-Cell Count and Diarrhea in HIV Patients. Korean J. Parasitol. 2015, 53, 705-712. [CrossRef]

102. Berenji, F.; Sarvghad, M.R.; Fata, A.; Hosseininejad, Z.; Saremi, E.; Ganjbakhsh, M.; Jahanparvar, R.I. A Study of the Prevalence of Intestinal Parasitic Infection in HIV Positive Individuals in Mashhad, Northeast Iran. Jundishapur J. Microbiol. 2010, 3, 61-65.

103. Yosefi, F.; Rahdar, M.; Alavi, S.M.; Samany, A. A Study on Prevalence of Gastrointestinal Parasitic Infections in HIV (+) Patients Referred to Ahvaz Razi Hospital in 2008-2009. Jundishapur J. Microbiol. 2012, 5, 424-426. [CrossRef]

104. Agholi, M.; Hatam, G.R.; Motazedian, M.H. HIV/AIDS-Associated Opportunistic Protozoal Diarrhea. Aids Res. Hum. Retrovir. 2013, 29, 35-41. [CrossRef] [PubMed]

105. Masoumi-Asl, H.; Khanaliha, K.; Bokharaei-Salim, F.; Esteghamati, A.; Kalantari, S.; Hosseinyrad, M. Enteric Opportunistic Infection and the Impact of Antiretroviral Therapy among HIV/AIDS Patients from Tehran, Iran. Iran. J. Public Health 2019, 48, 730-739. [CrossRef] [PubMed]

106. Anvari-Tafti, M.H.; Eslami, G.; Teimourzadeh-Baboli, A.; Ghafourzadeh, M. Food-Borne Protozoan Infection in HIV /AIDS $^{+}$ Patients and Healthy Individuals: A Case-Control Study in Iran. J. Food Qual. Hazards Control 2016, 3, 93-96.

107. Piranshahi, A.R.; Tavalla, M.; Khademvatan, S. Genomic Analysis of Blastocystis hominis Isolates in Patients with HIV-Positive Using Locus SSU-RDNA. J. Parasit. Dis. 2018, 42, 28-33. [CrossRef]

108. Paboriboune, P.; Phoumindr, N.; Borel, E.; Sourinphoumy, K.; Phaxayaseng, S.; Luangkhot, E.; Sengphilom, B.; Vansilalom, Y.; Odermatt, P.; Delaporte, E.; et al. Intestinal Parasitic Infections in HIV-Infected Patients, Lao People's Democratic Republic. PLoS ONE 2014, 9, e91452. [CrossRef]

109. Sherchan, J.B.; Ohara, H.; Sakurada, S.; Basnet, A.; Tandukar, S.; Sherchand, J.B.; Bam, D.S. Enteric Opportunistic Parasitic Infections among HIV-Seropositive Patients in Kathmandu, Nepal. Kathmandu Univ. Med. J. 2012, 10, 14-17. [CrossRef] [PubMed]

110. Ghimire, A.; Bhandari, S.; Tandukar, S.; Amatya, J.; Bhandari, D.; Sherchand, J.B. Enteric Parasitic Infection among HIV-Infected Patients Visiting Tribhuvan University Teaching Hospital, Nepal. BMC Res. Notes 2016, 9, 204. [CrossRef]

111. Zorbozan, O.; Quliyeva, G.; Tunalı, V.; Özbilgin, A.; Turgay, N.; Gökengin, A.D. Intestinal Protozoa in Hiv-Infected Patients: A Retrospective Analysis. Turk. Parazitolojii Derg. 2018, 42, 187-190. [CrossRef]

112. Davis, N.A.; Islamova, Z.I.; Giiasov, K.Z.; Badalova, N.S.; Takhtokhodzhaeva, G.R.; Latipov, R.R.; Osipova, S.O. Blastocystis hominis and Nonpathogenic Enteric Protozoa in Patients with Pulmonary Tuberculosis and Those with HIV Infection. Med. Parazitol. 2010, 3, 8-11.

113. Taghipour, A.; Javanmard, E.; Mirjalali, H.; Haghighi, A.; Tabarsi, P.; Sohrabi, M.R.; Zali, M.R. Blastocystis Subtype 1 (Allele 4); Predominant Subtype among Tuberculosis Patients in Iran. Comp. Immunol. Microbiol. Infect. Dis. 2019, 65, 201-206. [CrossRef] [PubMed]

114. Taghipour, A.; Tabarsi, P.; Sohrabi, M.R.; Riahi, S.M.; Rostami, A.; Mirjalali, H.; Malih, N.; Haghighi, A. Frequency, Associated Factors and Clinical Symptoms of Intestinal Parasites among Tuberculosis and Non-Tuberculosis Groups in Iran: A Comparative Cross-Sectional Study. Trans. R. Soc. Trop. Med. Hyg. 2019, 113, 234-241. [CrossRef]

115. Li, X.-X.; Chen, J.-X.; Wang, L.-X.; Tian, L.-G.; Zhang, Y.-P.; Dong, S.-P.; Hu, X.-G.; Liu, J.; Wang, F.-F.; Wang, Y.; et al. Intestinal Parasite Co-Infection among Pulmonary Tuberculosis Cases without Human Immunodeficiency Virus Infection in a Rural County in China. Am. J. Trop. Med. Hyg. 2014, 90, 106-113. [CrossRef] [PubMed] 
116. Li, X.-X.; Chen, J.-X.; Wang, L.-X.; Tian, L.-G.; Zhang, Y.-P.; Dong, S.-P.; Hu, X.-G.; Liu, J.; Wang, F.-F.; Wang, Y.; et al. Prevalence and Risk Factors of Intestinal Protozoan and Helminth Infections among Pulmonary Tuberculosis Patients without HIV Infection in a Rural County in P. R. China. Acta Trop. 2015, 149, 19-26. [CrossRef] [PubMed]

117. Taghipour, A.; Azimi, T.; Javanmard, E.; Pormohammad, A.; Olfatifar, M.; Rostami, A.; Tabarsi, P.; Sohrabi, M.R.; Mirjalali, H.; Haghighi, A. Immunocompromised Patients with Pulmonary Tuberculosis; a Susceptible Group to Intestinal Parasites. Gastroenterol. Hepatol. Bed Bench 2018, 11, S134-S139. [PubMed]

118. Azami, M.; Sharifi, M.; Hejazi, S.H.; Tazhibi, M. Intestinal Parasitic Infections in Renal Transplant Recipients. Braz. J. Infect. Dis. 2010, 14, 15-18. [CrossRef]

119. Idris, N.S.; Dwipoerwantoro, P.G.; Kurniawan, A.; Said, M. Intestinal Parasitic Infection of Immunocompromised Children with Diarrhea: Clinical Profile and Therapeutic Response. J. Infect. Dev. Ctries. 2010, 4, 309-317. [CrossRef] [PubMed]

120. Caner, A.; Zorbozan, O.; Tunalı, V.; Kantar, M.; Aydoğdu, S.; Aksoylar, S.; Gürüz, Y.; Turgay, N. Intestinal Protozoan Parasitic Infections in Immunocompromised Child Patients with Diarrhea. Jpn. J. Infect. Dis. 2020, 73, 187-192. [CrossRef] [PubMed]

121. Rasti, S.; Hassanzadeh, M.; Hooshyar, H.; Momen-Heravi, M.; Mousavi, S.G.A.; Abdoli, A. Intestinal Parasitic Infections in Different Groups of Immunocompromised Patients in Kashan and Qom Cities, Central Iran. Scand. J. Gastroenterol. 2017, 52, 738-741. [CrossRef]

122. Izadi, S.; Ghayour-Najafabadi, Z.; Yavari, M.; Mohaghegh, M.-A.; Wannigama, D.L.; Moslemzadeh, H.-R.; Azami, M.; Hejazi, S.-H. Intestinal Parasites Associated with Opportunistic Coccidial Infections among Immunocompromised Individuals in Central Iran: A Cross Sectional Study. Arch. Clin. Infect. Dis. 2019, 14, e79701. [CrossRef]

123. Esteghamati, A.; Khanaliha, K.; Bokharaei-Salim, F.; Sayyahfar, S.; Ghaderipour, M. Prevalence of Intestinal Parasitic Infection in Cancer, Organ Transplant and Primary Immunodeficiency Patients in Tehran, Iran. Asian Pac. J. Cancer Prev. 2019, 20, 495-501. [CrossRef]

124. Mirzaei, L.; Ashrafi, K.; Atrkar Roushan, Z.; Mahmoudi, M.R.; Shenavar Masooleh, I.; Rahmati, B.; Saadat, F.; Mirjalali, H.; Sharifdini, M. Strongyloides Stercoralis and Other Intestinal Parasites in Patients Receiving Immunosuppressive Drugs in Northern Iran: A Closer Look at Risk Factors. Epidemiol. Health 2021, 43, e2021009. [CrossRef]

125. Al-Megrin, W.A.I. Intestinal Parasites Infection among Immunocompromised Patients in Riyadh, Saudi Arabia. Pak. J. Biol. Sci. 2010, 13, 390-394. [CrossRef] [PubMed]

126. Uysal, S.; Tunalı, V.; Akdur Öztürk, E.; Ardeniz, Ö.; Işıkgöz Taşbakan, M.; Pullukçu, H.; Özensoy Töz, S.; Turgay, N.; Arda, B. Incidence of Parasitic Diarrhea in Patients with Common Variable Immune Deficiency. Turk. Parazitolojii Derg. 2016, 40, 67-71. [CrossRef]

127. Maçin, S.; Kaya, F.; Çağdaş, D.; Hizarcioglu-Gulsen, H.; Saltik-Temizel, I.N.; Tezcan, İ.; Demir, H.; Ergüven, S.; Akyön, Y. Detection of Parasites in Children with Chronic Diarrhea. Pediatr. Int. 2016, 58, 531-533. [CrossRef]

128. Asadi, L.; Pourlak, T.; Ahmadi, B.; Aghamali, M.; Asgharzadeh, M.; Aghazadeh, M.; Zeinalzadeh, E.; Kafil, H.S. Etiological Agents of Pediatric Diarrhea in Ardebil, Northwestern Iran. Arch. Pediatr. Infect. Dis. 2018, 6, e11771. [CrossRef]

129. Boughattas, S.; Behnke, J.M.; Al-Ansari, K.; Sharma, A.; Abu-Alainin, W.; Al-Thani, A.; Abu-Madi, M.A. Molecular Analysis of the Enteric Protozoa Associated with Acute Diarrhea in Hospitalized Children. Front. Cell. Infect. Microbiol. 2017, 7, 343. [CrossRef]

130. Dahal, M.; Dahal, R.H.; Chaudhary, D.K. Prevalence of Cyclospora Cayetanensis and Other Enteropathogen among Children under the Age of 15 Years in Biratnagar, Nepal. Asian Pac. J. Trop. Dis. 2017, 7, 75-79. [CrossRef]

131. Khalili, B.; Khani, M.R.; Taghipour, S. Blastocystis hominis Infection among Hospitalized Children Due to Diarrhea in Hajar Hospital, Shahre-Kord, Iran. Arch. Clin. Infect. Dis. 2012, 7, 52-55. [CrossRef]

132. Kiani, H.; Haghighi, A.; Salehi, R.; Azargashb, E. Distribution and Risk Factors Associated with Intestinal Parasite Infections among Children with Gastrointestinal Disorders. Gastroenterol. Hepatol. Bed Bench 2016, 9, S80-S87.

133. Awae, N.; Pipatsatitpong, D.; Mungthin, M.; Ruang-Areerate, T.; Leelayoova, S.; Aunpad, R.; Suwanvattana, P.; Thawornwan, U. Prevalence of and Risk Factors Associated with Parasitic, Bacterial and Viral Infections among Children with Gastrointestinal Illness in Bamrasnaradura Infectious Diseases Institute. Sci. Technol. Asia 2018, 23, 44-51. [CrossRef]

134. Sigidaev, A.S.; Kozlov, S.S.; Tarasova, E.A.; Suvorova, M.A. Investigation of the Genetic Profile of Blastocystis Species in Saint Petersburg Residents with Gastrointestinal Tract Diseases in Different Age Groups. Med. Parazitol. 2013, 4, 19-23.

135. Oyofo, B.A.; Subekti, D.; Tjaniadi, P.; Machpud, N.; Komalarini, S.; Setiawan, B.; Simanjuntak, C.; Punjabi, N.; Corwin, A.L.; Wasfy, M.; et al. Enteropathogens Associated with Acute Diarrhea in Community and Hospital Patients in Jakarta, Indonesia. Fems Immunol. Med. Microbiol. 2002, 34, 139-146. [CrossRef]

136. Zhang, S.-X.; Yang, C.-L.; Gu, W.-P.; Ai, L.; Serrano, E.; Yang, P.; Zhou, X.; Li, S.-Z.; Lv, S.; Dang, Z.-S.; et al. Case-Control Study of Diarrheal Disease Etiology in Individuals over 5 Years in Southwest China. Gut Pathog. 2016, 8, 58. [CrossRef]

137. Won, E.J.; Kim, S.H.; Kee, S.J.; Shin, J.H.; Suh, S.P.; Chai, J.Y.; Ryang, D.W.; Shin, M.G. Multiplex Real-Time PCR Assay Targeting Eight Parasites Customized to the Korean Population: Potential Use for Detection in Diarrheal Stool Samples from Gastroenteritis Patients. PLoS ONE 2016, 11, 0166957. [CrossRef] [PubMed]

138. Jalallou, N.; Iravani, S.; Rezaeian, M.; Alinaghizade, A.; Mirjalali, H. Subtypes Distribution and Frequency of Blastocystis sp. Isolated from Diarrheic and Non-Diarrheic Patients. Iran. J. Parasitol. 2017, 12, 63-68. [PubMed]

139. Najafi, A.; Mirzaei, A.; kermanjani, A.; Abdi, J.; Ghaderi, A.; Naserifar, R. Molecular Identification of Entamoeba Histolytica from Stool Samples of Ilam, Iran. Comp. Immunol. Microbiol. Infect. Dis. 2019, 63, 145-147. [CrossRef] [PubMed]

140. Kiani, H.; Haghighi, A.; Rostami, A.; Azargashb, E.; Seyyed Tabaei, S.J.; Solgi, A.; Zebardast, N. Prevalence, Risk Factors and Symptoms Associated to Intestinal Parasite Infections among Patients with Gastrointestinal Disorders in Nahavand, Western Iran. Rev. Inst. Med. Trop. Sao Paulo 2016, 58, 42. [CrossRef] [PubMed] 
141. Moosavi, A.; Haghighi, A.; Mojarad, E.N.; Zayeri, F.; Alebouyeh, M.; Khazan, H.; Kazemi, B.; Zali, M.R. Genetic Variability of Blastocystis sp. Isolated from Symptomatic and Asymptomatic Individuals in Iran. Parasitol. Res. 2012, 111, 2311-2315. [CrossRef] [PubMed]

142. Shahbazi, A.; Aboualsoltani, N.; Bazmani, A.; Khanmohammadi, M.; Aboualsoltani, F.; Fallah, E. PCR-Based Subtyping of Blastocystis Isolates from Symptomatic and Asymptomatic Patients in North-West of Iran. J. Pure Appl. Microbiol. 2013, 7, $2957-2963$.

143. Mirjalali, H.; Abbasi, M.R.; Naderi, N.; Hasani, Z.; Mirsamadi, E.S.; Stensvold, C.R.; Balaii, H.; Asadzadeh Aghdaei, H.; Zali, M.R. Distribution and Phylogenetic Analysis of Blastocystis sp. Subtypes Isolated from IBD Patients and Healthy Individuals in Iran. Eur. J. Clin. Microbiol. Infect. Dis. 2017, 36, 2335-2342. [CrossRef]

144. Kesuma, Y.; Firmansyah, A.; Bardosono, S.; Sari, I.P.; Kurniawan, A. Blastocystis ST-1 Is Associated with Irritable Bowel SyndromeDiarrhea (IBS-D) in Indonesian Adolescences. Parasite Epidemiol. Control 2019, 6, e00112. [CrossRef] [PubMed]

145. Das, R.; Khalil, S.; Mirdha, B.R.; Makharia, G.K.; Dattagupta, S.; Chaudhry, R. Molecular Characterization and Subtyping of Blastocystis species in Irritable Bowel Syndrome Patients from North India. PLoS ONE 2016, 11, 0147055. [CrossRef]

146. Shafiei, Z.; Esfandiari, F.; Sarkari, B.; Rezaei, Z.; Fatahi, M.R.; Hosseini Asl, S.M.K. Parasitic Infections in Irritable Bowel Syndrome Patients: Evidence to Propose a Possible Link, Based on a Case-Control Study in the South of Iran. BMC Res. Notes 2020, 13, 264. [CrossRef]

147. Khademvatan, S.; Masjedizadeh, R.; Rahim, F.; Mahbodfar, H.; Salehi, R.; Yousefi-Razin, E.; Foroutan, M. Blastocystis and Irritable Bowel Syndrome: Frequency and Subtypes from Iranian Patients. Parasitol. Int. 2017, 66, 142-145. [CrossRef]

148. Sayal, R.A.; Hameed, S.; Faisal, M.M. Evaluation of IL-5 Concentration Level in Irritable Bowel Syndrome Patients That Suffering from Blastocystis Infection in Al-Najaf Province. Eur. J. Mol. Clin. Med. 2020, 7, 3807-3817.

149. Surangsrirat, S.; Thamrongwittawatpong, L.; Piyaniran, W.; Naaglor, T.; Khoprasert, C.; Taamasri, P.; Mungthin, M.; Leelayoova, S. Assessment of the Association between Blastocystis Infection and Irritable Bowel Syndrome. J. Med. Assoc. Thai. 2010, 93 (Suppl. 6), S119-S124.

150. Merza, A.S.; Mohammed Al-Saeed, A.T.; Najeeb, M.K. Comparison of The Efficiency of Different Techniques for The Detection Of Blastocystis hominis In Patients Attending Hospitals Of Duhok City, Kurdistan Region, Iraq. Biochem. Cell. Arch. 2020, 20, 4421-4425.

151. Mutlag, S.K.; Ahmed, N.A.; Abbas, S.K. Investigation the Role of Blastocystis hominis Effect on The Levels of Il-10, Il-18 And Hematological Parameters. Biochem. Cell. Arch. 2019, 19, 3887-3892. [CrossRef]

152. Sanpool, O.; Laoraksawong, P.; Janwan, P.; Intapan, P.M.; Sawanyawisuth, K.; Thanchomnang, T.; Changtrakul, Y.; Maleewong, W. Genetic Subtypes of Blastocystis Isolated from Thai Hospitalized Patients in Northeastern Thailand. Southeast Asian J. Trop. Med. Public Health 2015, 46, 184-190. [PubMed]

153. Koltas, I.S.; Elgun, G.; Eroglu, F.; Demirkazık, M. The Importance of Real-Time Polymerase Chain Reaction Method in Diagnosis of Intestinal Parasites in Cases with Diarrhea. Trop. Biomed. 2017, 34, 895-902.

154. Aykur, M.; Calıskan Kurt, C.; Dirim Erdogan, D.; Biray Avcı, C.; Vardar, R.; Aydemir, S.; Girginkardeşler, N.; Gündüz, C.; Dagci, H. Investigation of Dientamoeba fragilis Prevalence and Evaluation of Sociodemographic and Clinical Features in Patients with Gastrointestinal Symptoms. Acta Parasitol. 2019, 64, 162-170. [CrossRef] [PubMed]

155. Usluca, S.; Inceboz, T.; Over, L.; Tuncay, S.; Yalçin, G.; Arcak, S.S.; Ozkoç, S.; Aksoy, U.; Akisü, C. [The Distribution of Intestinal Parasites Detected in The Dokuz Eylul University Medical Faculty Hospital between 2005 and 2008.]. Dokuz Eylül Universitesi Tip Fakültesi Araştirma ve Uygulama Hastanesi'nde 2005-2008 Yillari Arasinda Saptanan Bağirsak Pa. Turk. Parazitolojii Derg. 2010, 34, 27-31.

156. Cekin, A.H.; Cekin, Y.; Adakan, Y.; Tasdemir, E.; Koclar, F.G.; Yolcular, B.O. Blastocystosis in Patients with Gastrointestinal Symptoms: A Case-Control Study. BMC Gastroenterol. 2012, 12, 122. [CrossRef] [PubMed]

157. Beiromvand, M.; Hashemi, S.J.; Arjmand, R.; Sadjadei, N.; Hardanipasand, L. Comparative Prevalence of Blastocystis in Patients with the Irritable Bowel Syndrome and Healthy Individuals: A Case Control Study. Jundishapur J. Microbiol. 2017, 10, e13572. [CrossRef]

158. Feurle, G.E.; Moos, V.; Landt, O.; Corcoran, C.; Reischl, U.; Maiwald, M. Tropheryma whipplei in Feces of Patients with Diarrhea in 3 Locations on Different Continents. Emerg. Infect. Dis. 2021, 27, 932-935. [CrossRef]

159. Hawash, Y.A.; Ismail, K.A.; Saber, T.; Eed, E.M.; Khalifa, A.S.; Alsharif, K.F.; Alghamdi, S.A. Dientamoeba fragilis Infection in Patients with Digestive and Non-Digestive Symptoms: A Case-Control Study. Korean J. Parasitol. 2020, 58, 129-134. [CrossRef]

160. Alver, O.; Oral, B.; Töre, O. The Distribution of Intestinal Parasites Detected in the Uludag University Medical School Hospital between 2005 and 2008 Uludağ Üniversitesi Tip Fakültesi Hastanesine 2005-2008 Yillari Arasinda Başvuran Kişilerde Saptanan Bağirsak Parazitlerinin Daği. Turk. Parazitolojii Derg. 2011, 35, 194-198. [CrossRef]

161. Inceboz, T.; Usluca, S.; Over, L.; Yalçin, G.; Tuncay, S.; Ozkoç, S. The Epidemiology Research of Blastocystis hominis in the Dokuz Eylül University Medical Faculty Hospital between 2005 and 2009 Dokuz Eylül Üniversitesi Hastanesi'ne 2005-2009 Yillari Arasinda Başvuran Olgularda Blastocystis hominis Epidemiyolojisinin. Turk. Parazitolojii Derg. 2011, 35, 72-76. [CrossRef]

162. Rostami Nejad, M.; Nazemalhosseini Mojarad, E.; Dabiri, H.; Nochi, Z.; Pourhoseingholi, M.A.; Sahebekhtiari, N.; Habibi, M.; Zali, M.R. A Case-Control Study of Blastocystis hominis among Iranian Population. East. Afr. J. Public Health 2010, 7, 101-104.

163. Haider, S.S.; Baqai, R.; Qureshi, F.M.; Boorom, K. Blastocystis spp., Cryptosporidium spp., and Entamoeba histolytica Exhibit Similar Symptomatic and Epidemiological Patterns in Healthcare-Seeking Patients in Karachi. Parasitol. Res. 2012, 111, 1357-1368. [CrossRef]

164. Sakalar, Ç.; Kandemir, I.; Uyar, Y.; Kuk, S.; Gürbüz, E.; Yazar, S. Polymerase Chain Reaction Based Subtyping of Blastocystis spp. Isolates from Symptomatic Patients in Turkey I Türkiye'deki Semptomatik Hastalardan Elde Edilen Blastocystis spp. İzolatlarinin Polimeraz Zincir Reaksiyonu Ile Alt Tiplendirilmesi. Turk. Klin. J. Med. Sci. 2013, 33, 1064-1068. [CrossRef]

165. Sharif, M.; Daryani, A.; Asgarian, F.; Nasrolahei, M. Intestinal Parasitic Infections among Intellectual Disability Children in Rehabilitation Centers of Northern Iran. Res. Dev. Disabil. 2010, 31, 924-928. [CrossRef] [PubMed] 
166. Hazrati Tappeh, K.H.; Mohammadzadeh, H.; Nejad Rahim, R.; Barazesh, A.; Khashaveh, S.H.; Taherkhani, H. Prevalence of Intestinal Parasitic Infections among Mentally Disabled Children and Adults of Urmia, Iran. Iran. J. Parasitol. 2010, 5, 60-64.

167. Khalili, B.; Imani, R.; Boostani, S. Intestinal Parasitic Infections in Chronic Psychiatric Patients in Sina Hospital Shahre-Kord, Iran. Jundishapur J. Microbiol. 2013, 6, 252-255. [CrossRef]

168. Saeidinia, A.; Tavakoli, I.; Naghipour, M.R.; Rahmati, B.; Ghavami Lahiji, H.; Salkhori, O.; Ashrafi, K. Prevalence of Strongyloides stercoralis and Other Intestinal Parasites among Institutionalized Mentally Disabled Individuals in Rasht, Northern Iran. Iran. J. Parasitol. 2016, 11, 527-533.

169. Shokri, A.; Sarasiabi, K.S.; Teshnizi, S.H.; Mahmoodi, H. Prevalence of Strongyloides stercoralis and Other Intestinal Parasitic Infections among Mentally Retarded Residents in Central Institution of Southern Iran. Asian Pac. J. Trop. Biomed. 2012, 2, 88-91. [CrossRef]

170. Rasti, S.; Arbabi, M.; Hooshyar, H. High Prevalence of Entamoeba histolytica and Enterobius vermicularis among Elderly and Mentally Retarded Residence in Golabchi Center, Kashan, Iran 2006-2007. Jundishapur J. Microbiol. 2012, 5, 585-589. [CrossRef]

171. Mohammadi-Meskin, V.; Hamedi, Y.; Heydarihengami, M.; Eftekhar, E.; Shamseddin, J.; Sharifisarasiabi, K. Intestinal Parasitic Infections in Mental Retardation Center of Bandar Abbas, Southern Iran. Iran. J. Parasitol. 2019, 14, 318-325. [CrossRef]

172. Sheikh, S.; Asghari, A.; Sadraei, J.; Pirestani, M.; Zare, M. Blastocystis sp. Subtype 9: As the First Reported Subtype in Patients with Schizophrenia in Iran. SN Compr. Clin. Med. 2020, 2, 633-639. [CrossRef]

173. Zhu, W.; Wei, Z.; Li, Q.; Lin, Y.; Yang, H.; Li, W. Prevalence and Subtype Diversity of Blastocystis in Human and Nonhuman Primates in North China. Parasitol. Res. 2020, 119, 2719-2725. [CrossRef] [PubMed]

174. Kang, J.-M.; Li, Y.-T.; Chen, R.; Yu, Y.-F.; Li, X.-T.; Wu, X.-P.; Chu, Y.-H.; Chen, J.-X.; Zhang, S.-X.; Tian, L.-G. Prevalence and Risk Factors of Blastocystis hominis Infection in Inpatients in Jiangjin District, Chongqing City. Chin. J. Schistosomiasis Control 2019, 31, 479-485. [CrossRef]

175. Abdipour, Y.; Khazan, H.; Azargashb, E.; Mahmoudi, M.R.; Farahnak, A.; Rostami, A. Prevalence of Intestinal Parasitic Infections among Individuals Referred to the Medical Centers of Coastal Cities, Guilan Province, Northern Iran, 2015-2017. Iran. J. Public Health 2020, 49, 1157-1163.

176. Bahrami, F.; Haghighi, A.; Zamini, G.; Khademerfan, M. Molecular Evidence for Zoonotic Transmission of Blastocystis Subtypes in Kurdistan Province, West of Iran. Ann. Parasitol. 2020, 66, 19-25. [CrossRef] [PubMed]

177. Gholipoor, Z.; Khazan, H.; Azargashb, E.; Youssefi, M.R.; Rostami, A. Prevalence and Risk Factors of Intestinal Parasite Infections in Mazandaran Province, North of Iran. Clin. Epidemiol. Glob. Health 2020, 8, 17-20. [CrossRef]

178. Viesy, S.; Abdi, J.; Rezaei, Z. What Are Hidden Facts behind Intestinal Parasitic Infections in Ilam City? Infect. Disord. Drug Targets 2019, 19, 278-281. [CrossRef]

179. Badparva, E.; Sadraee, J.; Kheirandish, F.; Frouzandeh, M. Genetic Diversity of Human Blastocystis Isolates in Khorramabad, Central Iran. Iran. J. Parasitol. 2014, 9, 44-49. [PubMed]

180. Shaker, D.; Anvari, D.; Hosseini, S.A.; Fakhar, M.; Mardani, A.; Ziaei Hezarjaribi, H.; Gholami, S.; Gholami, S. Frequency and Genetic Diversity of Blastocystis Subtypes among Patients Attending to Health Centers in Mazandaran, Northern Iran. J. Parasit. Dis. 2019, 43, 537-543. [CrossRef]

181. Haghighi, L.; Talebnia, S.E.; Mikaeili, F.; Asgari, Q.; Gholizadeh, F.; Zomorodian, K. Prevalence and Subtype Identification of Blastocystis Isolated from Human in Shiraz City, Southern Iran. Clin. Epidemiol. Glob. Health 2020, 8, 840-844. [CrossRef]

182. Shaker, D.; Fakhar, M.; Ziaei, H.; Hosseini, S.A.; Gholami, S. Prevalence of Blastocystis hominis in Individuals Attending Sari Health Centers, 2014. J. Maz. Univ. Med. Sci. 2017, 27, 143-147.

183. Tork, M.; Yazdani-Charati, J.; Sharif, M.; Dryani, A.; Nazar, I.; Pagheh, A.S.; Hosseini, S.-A. Association between Geographical Risk Factors and Intestinal Parasites in West of Mazandaran Province Using Geographic Information System. J. Maz. Univ. Med. Sci. 2017, 27, 124-132.

184. Asfaram, S.; Daryani, A.; Sarvi, S.; Pagheh, A.S.; Hosseini, S.A.; Saberi, R.; Hoseiny, S.M.; Soosaraei, M.; Sharif, M. Geospatial Analysis and Epidemiological Aspects of Human Infections with Blastocystis hominis in Mazandaran Province, Northern Iran. Epidemiol. Health 2019, 41, e2019009. [CrossRef]

185. Bafghi, A.F.; Hosseini, R.; Mollaei, H.R.; Barzegar, K. Geno-Typing and Comparison of Conventional and Molecular Diagnostic Techniques for Detection of Blastocystis on Health Centers in Kerman Iran. Epidemiol. Health 2021, 8, $10-16$.

186. Karimazar, M.; Rezaeian, S.; Ebrahimipour, M.; Ranjbarpour, A.; Madanipour, H.; Javan, S.; Najjari, M. Prevalence and Time-Trend Analysis of Intestinal Parasitic Infections in North-Central Iran, 2012-2016. Trop. Biomed. 2019, 36, 103-113.

187. Abdul Ridha, A.A.F.; Faieq, Z.A. Epidemiology and Clinical Characteristics Associated with Blastocystis hominis in More Than Ten Years Infections in Wasit Province/Iraq. J. Phys. Conf. Ser. 2021, 1818, 012029. [CrossRef]

188. Salehi, R.; Haghighi, A.; Stensvold, C.R.; Kheirandish, F.; Azargashb, E.; Raeghi, S.; Kohansal, C.; Bahrami, F. Prevalence and Subtype Identification of Blastocystis Isolated from Humans in Ahvaz, Southwestern Iran. Gastroenterol. Hepatol. Bed Bench 2017, 10, 235-241. [CrossRef] [PubMed]

189. Khademvatan, S.; Masjedizadeh, R.; Yousefi-Razin, E.; Mahbodfar, H.; Rahim, F.; Yousefi, E.; Foroutan, M. PCR-Based Molecular Characterization of Blastocystis hominis Subtypes in Southwest of Iran. J. Infect. Public Health 2018, 11, 43-47. [CrossRef] [PubMed]

190. Sardarian, K.; Hajilooi, M.; Maghsood, A.; Moghimbeigi, A.; Alikhani, M. A Study of the Genetic Variability of Blastocystis hominis Isolates in Hamadan, West of Iran. Jundishapur J. Microbiol. 2012, 5, 555-559. [CrossRef]

191. Hamidi, N.; Meamar, A.R.; Akhlaghi, L.; Rampisheh, Z.; Razmjou, E. Dientamoeba fragilis Diagnosis by Fecal Screening: Relative Effectiveness of Traditional Techniques and Molecular Methods. J. Infect. Dev. Ctries. 2018, 12, 52-59. [CrossRef] [PubMed] 
192. Javadi, R.A.; Kazemi, F.; Fallahizadeh, S.; Arjmand, R. The Prevalence of Intestinal Parasitic Infections in Ahvaz, Southwest of Iran, during 2007-2017. Iran. J. Public Health 2019, 48, 2070-2073.

193. Delshad, A.; Saraei, M.; Alizadeh, S.A.; Niaraki, S.R.; Alipour, M.; Hosseinbigi, B.; Bozorgomid, A.; Hajialilo, E. Distribution and Molecular Analysis of Blastocystis Subtypes from Gastrointestinal Symptomatic and Asymptomatic Patients in Iran. Afr. Health Sci. 2020, 20, 1179-1189. [CrossRef] [PubMed]

194. Norouzi, P.; Mohaghegh, M.-A.; Ghorbani, M.; Mirzaii, M.; Abolhassani, M.; Mirbadie, S.-R. Investigating the Prevalence of Intestinal Parasites with an Emphasis on Strongyloides stercoralis Infection in Hospitalized Patients: A Regional Report from Iran. Ann. Parasitol. 2020, 66, 365-371. [CrossRef] [PubMed]

195. Sharifi, Y.; Abbasi, F.; Shahabi, S.; Zaraei, A.; Mikaeili, F.; Sarkari, B. Comparative Genotyping of Blastocystis Infecting Cattle and Human in the South of Iran. Comp. Immunol. Microbiol. Infect. Dis. 2020, 72, 101529. [CrossRef] [PubMed]

196. Salehi, M.; Mardaneh, J.; Niazkar, H.R.; Minooeianhaghighi, M.; Arshad, E.; Soleimani, F.; Mohammadzadeh, A. Prevalence and Subtype Analysis of Blastocystis hominis Isolated from Patients in the Northeast of Iran. J. Parasitol. Res. 2021, 2021, 8821885. [CrossRef] [PubMed]

197. Greige, S.; El Safadi, D.; Khaled, S.; Gantois, N.; Baydoun, M.; Chemaly, M.; Benamrouz-Vanneste, S.; Chabé, M.; Osman, M.; Certad, G.; et al. First Report on the Prevalence and Subtype Distribution of Blastocystis sp. in Dairy Cattle in Lebanon and Assessment of Zoonotic Transmission. Acta Trop. 2019, 194, 23-29. [CrossRef]

198. El Safadi, D.; Meloni, D.; Poirier, P.; Osman, M.; Cian, A.; Gaayeb, L.; Wawrzyniak, I.; Delbac, F.; El Alaoui, H.; Delhaes, L.; et al Short Report: Molecular Epidemiology of Blastocystis in Lebanon and Correlation between Subtype 1 and Gastrointestinal Symptoms. Am. J. Trop. Med. Hyg. 2013, 88, 1203-1206. [CrossRef]

199. Greige, S.; El Safadi, D.; Bécu, N.; Gantois, N.; Pereira, B.; Chabé, M.; Benamrouz-Vanneste, S.; Certad, G.; El Hage, R.; Chemaly, M.; et al. Prevalence and Subtype Distribution of Blastocystis sp. Isolates from Poultry in Lebanon and Evidence of Zoonotic Potential. Parasites Vectors 2018, 11, 1-10. [CrossRef]

200. Imam, A.; Altayyar, A.; Eltayeb, E.; Almushawa, Y. Frequency and Seasonality of Intestinal Parasitism in Qassim Region, Saudi Arabia. Pak. J. Med. Sci. 2012, 28, 10.

201. Hassen Amer, O.; Ashankyty, I.M.; Haouas, N.A.S. Prevalence of Intestinal Parasite Infections among Patients in Local Public Hospitals of Hail, Northwestern Saudi Arabia. Asian Pac. J. Trop. Med. 2016, 9, 44-48. [CrossRef]

202. Mohamed, R.T.; El-Bali, M.A.; Mohamed, A.A.; Abdel-Fatah, M.A.; El-Malky, M.A.; Mowafy, N.M.; Zaghlool, D.A.; Bakri, R.A.; Al-Harthi, S.A. Subtyping of Blastocystis sp. Isolated from Symptomatic and Asymptomatic Individuals in Makkah, Saudi Arabia. Parasites Vectors 2017, 10, 174. [CrossRef]

203. Laodim, P.; Intapan, P.M.; Sawanyawisuth, K.; Laummaunwai, P.; Maleewong, W. A Hospital-Based Study of Epidemiological and Clinical Data on Blastocystis hominis Infection. Foodborne Pathog. Dis. 2012, 9, 1077-1082. [CrossRef]

204. Jantermtor, S.; Pinlaor, P.; Sawadpanich, K.; Pinlaor, S.; Sangka, A.; Wilailuckana, C.; Wongsena, W.; Yoshikawa, H. Subtype Identification of Blastocystis spp. Isolated from Patients in a Major Hospital in Northeastern Thailand. Parasitol. Res. 2013, 112, 1781-1786. [CrossRef]

205. Polat, E.; Özdemir, S.; Sirekbasan, S. The Distribution of Intestinal Parasites in Patients Presenting to a University Hospital in Istanbul: A Seven-Year Retrospective Analysis. Turk. Parazitolojii Derg. 2020, 44, 139-142. [CrossRef] [PubMed]

206. Beyhan, Y.E.; Yilmaz, H.; Cengiz, Z.T.; Ekici, A. Clinical Significance and Prevalence of Blastocystis hominis in Van, Turkey. Saudi Med. J. 2015, 36, 1118-1121. [CrossRef] [PubMed]

207. Sarzhanov, F.; Köster, P.C.; Dogruman-Al, F.; Bailo, B.; Dashti, A.; Demirel-Kaya, F.; Carmena, D. Detection of Enteric Parasites and Molecular Characterization of Giardia Duodenalis and Blastocystis sp. In Patients Admitted to Hospital in Ankara, Turkey. Parasitology 2021, 148, 550-561. [CrossRef] [PubMed]

208. Selek, M.B.; Bektöre, B.; Karagöz, E.; Baylan, O.; Özyurt, M. Distribution of Parasites Detected in Stool Samples of Patients Admitted to Our Parasitology Laboratory during a Three-Year Period between 2012 and 2014. Turk. Parazitolojii Derg. 2016, 40, 137-140. [CrossRef] [PubMed]

209. Yaman, O.; Hamamci, B.; Cetinkaya, U.; Kaya, M.; Ateş, S.; Gözkenç, N.; Ozcan, H.; Yazar, L.; Yazar, S. The Investigation of Intestinal Parasites in Foreign High School Students Yabanci Uyruklu Lise Öğrencilerinde Intestinal Parazitlerin Araştirilmasi. Turk. Parazitolojii Derg. 2010, 34, 176-178.

210. Zhan, T.-Z.; Liu, T.; Shi, H.-H.; He, S.-S.; Yan, H.; Liu, D.-Y. PCR-Based Genotype Classification of Blastocystis hominis Isolates from College Students of Guangxi. Zhongguo Ji Sheng Chong Xue Yu Ji Sheng Chong Bing Za Zhi Chin. J. Parasitol. Parasit. Dis. 2014, 32, 209-211.

211. Fallahi, S.; Rostami, A.; Mohammadi, M.; Ebrahimzadeh, F.; Pournia, Y. Practical Parasitology Courses and Infection with Intestinal Parasites in Students. J. Infect. Public Health 2016, 9, 654-660. [CrossRef]

212. Srichaipon, N.; Nuchprayoon, S.; Charuchaibovorn, S.; Sukkapan, P.; Sanprasert, V. A Simple Genotyping Method for Rapid Differentiation of Blastocystis Subtypes and Subtype Distribution of Blastocystis spp. In Thailand. Pathogens 2019, 8, 38. [CrossRef]

213. Salemi, B.; Esteghamati, A.; Sayyahfar, S.; Bokharaei-Salim, F.; Keyvani, H.; Khanaliha, K. Frequency of Intestinal Parasitic Infection among Working Children in Tehran, Iran. Arch. Pediatr. Infect. Dis. 2019, 7, e93760. [CrossRef]

214. Pintong, A.-R.; Sunyanusin, S.; Prasertbun, R.; Mahittikorn, A.; Mori, H.; Changbunjong, T.; Komalamisra, C.; Sukthana, Y.; Popruk, S. Blastocystis Subtype 5: Predominant Subtype on Pig Farms, Thailand. Parasitol. Int. 2018, 67, 824-828. [CrossRef] [PubMed] 
215. Udonsom, R.; Prasertbun, R.; Mahittikorn, A.; Mori, H.; Changbunjong, T.; Komalamisra, C.; Pintong, A.-r.; Sukthana, Y.; Popruk, S. Blastocystis Infection and Subtype Distribution in Humans, Cattle, Goats, and Pigs in Central and Western Thailand. Infect. Genet. Evol. 2018, 65, 107-111. [CrossRef]

216. Kheirandish, F.; Tarahi, M.J.; Ezatpour, B. Prevalence of Intestinal Parasites among Food Handlers in Western Iran I La Prevalencia de Parásitos Intestinales Entre Los Manipuladores de Alimentos En El Oeste de Iŕn. Rev. Inst. Med. Trop. Sao Paulo 2014, 56, 111-114. [CrossRef]

217. Motazedian, M.H.; Najjari, M.; Ebrahimipour, M.; Asgari, Q.; Mojtabavi, S.; Mansouri, M. Prevalence of Intestinal Parasites among Food-Handlers in Shiraz, Iran. Iran. J. Parasitol. 2015, 10, 652-657.

218. Sharif, M.; Daryani, A.; Kia, E.; Rezaei, F.; Nasiri, M.; Nasrolahei, M. Prevalence of Intestinal Parasites among Food Handlers of Sari, Northern Iran I Prevalência de Parasitas Intestinais Entre Manipuladores de Alimentos de Sari, Norte Do Iran. Rev. Inst. Med. Trop. Sao Paulo 2015, 57, 139-144. [CrossRef]

219. Heydari-Hengami, M.; Hamedi, Y.; Najafi-Asl, M.; Sharifi-Sarasiabi, K. Prevalence of Intestinal Parasites in Food Handlers of Bandar Abbas, Southern Iran. Iran. J. Public Health 2018, 47, 111-118. [PubMed]

220. Khodabakhsh Arbat, S.; Hooshyar, H.; Arbabi, M.; Eslami, M.; Abani, B.; Poor Movayed, R. Prevalence of Intestinal Parasites among Food Handlers in Kashan, Central Iran, 2017-2018. J. Parasit. Dis. 2018, 42, 577-581. [CrossRef] [PubMed]

221. Shahnazi, M.; Sadeghi, M.; Saraei, M.; Alipour, M.; Hajialilo, E. Prevalence of Parasitic Intestinal Infections Among Food Handlers in Qazvin, İran. Turk. Parazitolojii Derg. 2019, 43, 16-20. [CrossRef]

222. Kheirandish, F.; Tarahi, M.J.; Haghighi, A.; Nazemalhosseini-Mojarad, E.; Kheirandish, M. Prevalence of Intestinal Parasites in Bakery Workers in Khorramabad, Lorestan Iran. Iran. J. Parasitol. 2011, 6, 76-83.

223. Abdel-Dayem, M.; Al Zou'bi, R.; Hani, R.B.; Amr, Z.S. Microbiological and Parasitological Investigation among Food Handlers in Hotels in the Dead Sea Area, Jordan. J. Microbiol. Immunol. Infect. 2014, 47, 377-380. [CrossRef] [PubMed]

224. Downs, J.W.; Putnam, S.D.; Rockabrand, D.M.; Okla, G.E.; Mostafa, M.; Monteville, M.R.; Antosek, L.E.; Herbst, J.; Tribble, D.R.; Riddle, M.S.; et al. A Cross-Sectional Analysis of Clinical Presentations of and Risk Factors for Enteric Protozoan Infections in an Active Duty Population during Operation Iraqi Freedom. Trop. Dis. Travel Med. Vaccines 2015, 1, 2. [CrossRef]

225. Kitvatanachai, S.; Rhongbutsri, P. Using Mini Parasep SF to Determine Intestinal Parasitic Infections Comparing to Conventional Methods in Gardener of Chanthaburi Province, Thailand. Asian Pac. J. Trop. Dis. 2017, 7, 596-600. [CrossRef]

226. Sangwalee, W.; Rattanapitoon, N.; Thanchomnang, T. Intestinal Parasitic Infections and Risk Factors among Myanmar Migrant Workers in Northeast Thailand. Asian Pac. J. Trop. Med. 2021, 14, 17. [CrossRef]

227. Abu-Madi, M.; Aly, M.; Behnke, J.M.; Clark, C.G.; Balkhy, H. The Distribution of Blastocystis Subtypes in Isolates from Qatar. Parasites Vectors 2015, 8, 465. [CrossRef]

228. Abu-Madi, M.; Boughattas, S.; Behnke, J.M.; Sharma, A.; Ismail, A. Coproscopy and Molecular Screening for Detection of Intestinal Protozoa. Parasites Vectors 2017, 10, 414. [CrossRef]

229. Abu-Madi, M.A.; Behnke, J.M.; Doiphode, S.H. Changing Trends in Intestinal Parasitic Infections among Long-Term-Residents and Settled Immigrants in Qatar. Parasites Vectors 2010, 3, 98. [CrossRef]

230. Abu-Madi, M.A.; Behnke, J.M.; Ismail, A.; Boughattas, S. Assessing the Burden of Intestinal Parasites Affecting Newly Arrived Immigrants in Qatar. Parasites Vectors 2016, 9, 619. [CrossRef]

231. Abu-Madi, M.A.; Behnke, J.M.; Boughattas, S.; Al-Thani, A.; Doiphode, S.H. A Decade of Intestinal Protozoan Epidemiology among Settled Immigrants in Qatar. BMC Infect. Dis. 2016, 16, 370. [CrossRef]

232. Abu-Madi, M.A.; Behnke, J.M.; Ismail, A.; Al-Olaqi, N.; Al-Zaher, K.; El-Ibrahim, R. Comparison of Intestinal Parasitic Infection in Newly Arrived and Resident Workers in Qatar. Parasites Vectors 2011, 4, 211. [CrossRef]

233. Wakid, M.H. Fecal Occult Blood Test and Gastrointestinal Parasitic Infection. J. Parasitol. Res. 2010, 2010, 1-4. [CrossRef]

234. Ahmed, M.A.; Alam-Eldin, Y.H.; Eltaweel, N.A.; Elmorsy, S. Intestinal Parasites Detected During Pre-Employment Stool Examination at Tertiary Health Care Center In Makkah, Kingdom Of Saudi Arabia. J. Egypt. Soc. Parasitol. 2015, 45, 367-373. [CrossRef]

235. Hsieh, M.-H.; Lin, W.-Y.; Dai, C.-Y.; Huang, J.-F.; Huang, C.-K.; Chien, H.-H.; Wang, C.-L.; Chung, W.-L.; Wu, J.-R.; Chen, E.-R.; et al. Intestinal Parasitic Infection Detected by Stool Examination in Foreign Laborers in Kaohsiung. Kaohsiung J. Med. Sci. 2010, 26, 136-143. [CrossRef]

236. Hsieh, M.-H.; Lin, Y.-Y.; Hsu, Y.-K.; Yang, J.-F.; Hsu, Y.-C.; Chen, W.-C.; Dai, C.-Y.; Yu, M.-L.; Ho, C.-K. Intestinal Parasitic Infections in Foreigners Detected by Stool Examination in Taiwan. Open Infect. Dis. J. 2011, 5, 135-141. [CrossRef]

237. Chen, L.H.; Lee, S.L.; Yang, C.C.; Lee, Y.M. Genetic Variability of Blastocystis hominis in Indonesian Immigrant Workers. J. Intern. Med. Taiwan 2014, 25, 199-205.

238. Karaman, U.; Turan, A.; Depecik, F.; Geçit, I.; Ozer, A.; Karci, E.; Karadan, M. [Frequency of Intestinal Parasites among Administrators and Workers in Sanitary and Non-Sanitary Institutions. Sihhi ve Gayri Sihhi Müesseselerdeki İsletmeci ve Çalişanlari ve Bağirsak Parazitlerinin Sikliği. Turk. Parazitolojii Derg. 2011, 35, 30-33. [CrossRef] [PubMed]

239. Sahimin, N.; Meor Termizi, F.H.; Rajamanikam, A.; Mohd Nazri, N.A.; Govind, S.K.; Mohd Zain, S.N. Prevalence and Subtypes of Blastocystis among Migrant Workers from Different Working Sectors in Peninsular Malaysia. Parasitol. Res. 2020, 119, 3555-3558. [CrossRef]

240. Noor, R.; Saha, S.R.; Rahman, F.; Munshi, S.K.; Uddin, M.A.; Rahman, M.M. Frequency of Opportunistic and Other Intestinal Parasitic Infections in Patients Infected with Human Immunodeficiency Virus in Bangladesh. Tzu Chi Med. J. 2012, 24, 191-195. [CrossRef] 
241. Schär, F.; Inpankaew, T.; Traub, R.J.; Khieu, V.; Dalsgaard, A.; Chimnoi, W.; Chhoun, C.; Sok, D.; Marti, H.; Muth, S.; et al. The Prevalence and Diversity of Intestinal Parasitic Infections in Humans and Domestic Animals in a Rural Cambodian Village. Parasitol. Int. 2014, 63, 597-603. [CrossRef]

242. Wang, W.; Owen, H.; Traub, R.J.; Cuttell, L.; Inpankaew, T.; Bielefeldt-Ohmann, H. Molecular Epidemiology of Blastocystis in Pigs and Their In-Contact Humans in Southeast Queensland, Australia, and Cambodia. Vet. Parasitol. 2014, 203, 264-269. [CrossRef]

243. He, S.S.; Wu, L.Y.; Liu, X.Q.; Shi, H.H.; Chen, Z.; Zhang, H.; Pang, C.Y.; Li, Y.M. Investigation on the Infection of Blastocystis hominis in Populations in Bama Yao Autonomous County of Guangxi. Zhongguo Ji Sheng Chong Xue Yu Ji Sheng Chong Bing Za Zhi Chin. J. Parasitol. Parasit. Dis. 2013, 31, 76-77.

244. Chen, S.; Zhang, Y.; Li, H.; Cai, Y.; Chen, J. Analysis on Parasitic Infection of Clinical Samples from Hospitals in Shanghai during 2011-2013. Zhongguo Ji Sheng Chong Xue Yu Ji Sheng Chong Bing Za Zhi Chin. J. Parasitol. Parasit. Dis. 2014, 32, 446-451.

245. Umar, M.; Chen, X.; Osman, Y.; Simayi, A.; Hou, Y.; Maimaitiyiming, Y.; Xiao, N. Epidemiological Survey on Human Intestinal Protozoa in Xinjiang Uygur Autonomous Region in 2015. Zhongguo Ji Sheng Chong Xue Yu Ji Sheng Chong Bing Za Zhi Chin. J. Parasitol. Parasit. Dis. 2016, 34, 361-365.

246. Jiang, L.-Z. Epidemic Status of Human Key Parasitic Diseases in Tongcheng City, Anhui Province. Chin. J. Schistosomiasis Control 2018, 30, 68-71. [CrossRef]

247. Zhang, Y.-L.; Zhu, Y.-K.; Chen, W.-Q.; Deng, Y.; Lin, X.-M.; Li, P.; Zhang, H.-W. Survey and Analysis of Epidemic Status of Principal Human Parasitosis in Ecological Region of Huaiyang Hills of Henan Province in 2015. Chin. J. Schistosomiasis Control 2017, 29. [CrossRef]

248. Gong, B.; Liu, X.; Wu, Y.; Xu, N.; Xu, M.; Yang, F.; Tong, L.; Zhou, K.; Cao, J.; Liu, A.; et al. Prevalence and Subtype Distribution of Blastocystis in Ethnic Minority Groups on Both Sides of the China-Myanmar Border, and Assessment of Risk Factors I révalence et Distribution Des Sous-Types de Blastocystis Dans Les Groupes de Minorités Ethniques Des. Parasite 2019, 26, 46. [CrossRef]

249. Deng, Y.; Zhang, S.; Ning, C.; Zhou, Y.; Teng, X.; Wu, X.; Chu, Y.; Yu, Y.; Chen, J.; Tian, L.; et al. Molecular Epidemiology and Risk Factors of Blastocystis sp. Infections among General Populations in Yunnan Province, Southwestern China. Risk Manag. Healthc. Policy 2020, 13, 1791-1801. [CrossRef]

250. Ma, L.; Qiao, H.; Wang, H.; Li, S.; Zhai, P.; Huang, J.; Guo, Y. Molecular Prevalence and Subtypes of Blastocystis sp. in Primates in Northern China. Transbound. Emerg. Dis. 2020, 67, 2789-2796. [CrossRef]

251. Seyer, A.; Karasartova, D.; Ruh, E.; Güreser, A.S.; Turgal, E.; Imir, T.; Taylan-Ozkan, A. Epidemiology and Prevalence of Blastocystis spp. in North Cyprus. Am. J. Trop. Med. Hyg. 2017, 96, 1164-1170. [CrossRef]

252. Padukone, S.; Mandal, J.; Rajkumari, N.; Bhat, B.; Swaminathan, R.; Parija, S. Detection of Blastocystis in Clinical Stool Specimens Using Three Different Methods and Morphological Examination in Jones' Medium. Trop. Parasitol. 2018, 8, 33-40. [CrossRef]

253. Lappan, R.; Classon, C.; Kumar, S.; Singh, O.P.; De Almeida, R.V.; Chakravarty, J.; Kumari, P.; Kansal, S.; Sundar, S.; Blackwell, J.M. Meta-Taxonomic Analysis of Prokaryotic and Eukaryotic Gut Flora in Stool Samples from Visceral Leishmaniasis Cases and Endemic Controls in Bihar State India. PLoS Negl. Trop. Dis. 2019, 13, e0007444. [CrossRef]

254. Wiria, A.E.; Hamid, F.; Wammes, L.J.; Prasetyani, M.A.; Dekkers, O.M.; May, L.; Kaisar, M.M.M.; Verweij, J.J.; Guigas, B.; Partono, F.; et al. Infection with Soil-Transmitted Helminths Is Associated with Increased Insulin Sensitivity. PLoS ONE 2015, 10, e0127746. [CrossRef]

255. Yulfi, H.; Rozi, M.F.; Andriyani, Y.; Darlan, D.M. Prevalence of Cryptosporidium Spp. and Blastocystis hominis in Faecal Samples among Diarrheic HIV Patients in Medan, Indonesia. Med. Glas. 2021, 18, 1-7. [CrossRef]

256. Sungkar, S.; Pohan, A.P.N.; Ramadani, A.; Albar, N.; Azizah, F.; Nugraha, A.R.A.; Wiria, A.E. Heavy Burden of Intestinal Parasite Infections in Kalena Rongo Village, a Rural Area in South West Sumba, Eastern Part of Indonesia: A Cross Sectional Study. BMC Public Health 2015, 15, 1296. [CrossRef] [PubMed]

257. Hayashi, E.; Tuda, J.; Imada, M.; Akao, N.; Fujita, K. The High Prevalence of Asymptomatic Toxocara Infection among Schoolchildren in Manado, Indonesia. Southeast Asian J. Trop Med. Public Health 2005, 36, 1399-1406. [PubMed]

258. Turgay, N.; Unver-Yolasiğmaz, A.; Oyur, T.; Bardak-Özcem, S.; Töz, S. Monthly Distribution of Intestinal Parasites Detected in a Part of Western Turkey between May 2009-April 2010-Results of Acid Fast and Modified Trichrome Staining Methods İzmir ve Çevresinde Bir Yilda (Mayis 2009-Nisan 2010) Saptanan Baǧirsak Parazit. Turk. Parazitolojii Derg. 2012, 36, 71-74. [CrossRef] [PubMed]

259. Mahmoudi, M.R.; Hasani, H.; Tsiami, A.; Ashrafi, K.; Johnson, P.; Sharifdini, M.; Karanis, P. Intestinal Protozoan and Helminthic Infections among Hemodialysis and Cancer Patients. Parasitol. Res. 2020, 119, 3053-3059. [CrossRef] [PubMed]

260. Khoshnood, S.; Rafiei, A.; Saki, J.; Alizadeh, K. Prevalence and Genotype Characterization of Blastocystis hominis among the Baghmalek People in Southwestern Iran in 2013-2014. Jundishapur J. Microbiol. 2015, 8, e23930. [CrossRef]

261. Pestehchian, N.; Nazari, M.; Haghighi, A.; Salehi, M.; Yosefi, H.; Khosravi, N. Prevalence of Intestinal Parasitic Infection among Inhabitants and Tribes of Chelgerd, Iran, 2008-2009. J. Clin. Diagn. Res. 2015, 9, LC01-LC04. [CrossRef]

262. Sadeghi, H.; Bakht, M.; Saghafi, H.; Shahsavari, T. Prevalence of Intestinal Parasites in a Population in Eghbalieh City from Qazvin Province, Iran. J. Parasit. Dis. 2015, 39, 126-129. [CrossRef] [PubMed]

263. Sadeghi, H.; Borji, H. A Survey of Intestinal Parasites in a Population in Qazvin, North of Iran. Asian Pac. J. Trop. Dis. 2015, 5 , 231-233. [CrossRef]

264. Badparva, E.; Kheirandish, F.; Ebrahimzade, F. Prevalence of Intestinal Parasites in Lorestan Province, West of Iran. Asian Pac. J. Trop. Dis. 2014, 4, S728-S732. [CrossRef]

265. Mahni, M.B.; Rezaeian, M.; Kia, E.B.; Raeisi, A.; Khanaliha, K.; Tarighi, F.; Kamranrashani, B. Prevalence of Intestinal Parasitic Infections in Jiroft, Kerman Province, Iran. Iran. J. Parasitol. 2016, 11, 232-238. 
266. Tork, M.; Sharif, M.; Charati, J.Y.; Nazar, I.; Hosseini, S.A. Prevalence of Intestinal Parasitic Infections and Associated Risk Factors in West of Mazandaran Province, Iran. J. Maz. Univ. Med. Sci. 2016, 25, 81-88.

267. Jafari, R.; Sharifi, F.; Bagherpour, B.; Safari, M. Prevalence of Intestinal Parasites in Isfahan City, Central Iran, 2014. J. Parasit. Dis. 2016, 40, 679-682. [CrossRef] [PubMed]

268. Hemmati, N.; Razmjou, E.; Hashemi-Hafshejani, S.; Motevalian, A.; Akhlaghi, L.; Meamar, A.R. Prevalence and Risk Factors of Human Intestinal Parasites in Roudehen, Tehran Province, Iran. Iran. J. Parasitol. 2017, 12, 364-373. [PubMed]

269. Riabi, T.R.; Haghighi, A.; Mirjalali, H.; Gol, S.M.A.; Karamati, S.A.; Ghasemian, M.; Monfared, A.B.; Aghamohammadi, E.; Zojaji, H. Study of Prevalence, Distribution and Clinical Significance of Blastocystis Isolated from Two Medical Centers in Iran. Gastroenterol. Hepatol. Bed Bench 2017, 10, S102-S107. [CrossRef]

270. Mardani Kataki, M.; Tavalla, M.; Beiromvand, M. Higher Prevalence of Blastocystis hominis in Healthy Individuals than Patients with Gastrointestinal Symptoms from Ahvaz, Southwestern Iran. Comp. Immunol. Microbiol. Infect. Dis. 2019, 65, 160-164. [CrossRef]

271. Abbaszadeh Afshar, M.J.; Barkhori Mehni, M.; Rezaeian, M.; Mohebali, M.; Baigi, V.; Amiri, S.; Amirshekari, M.B.; Hamidinia, R.; Samimi, M. Prevalence and Associated Risk Factors of Human Intestinal Parasitic Infections: A Population-Based Study in the Southeast of Kerman Province, Southeastern Iran. BMC Infect. Dis. 2020, 20, 12. [CrossRef]

272. Sobati, H. Epidemiological Study of Parasitic Infections in Bumusa Island, Hormozgan. Iran. J. Parasitol. 2020, 15, 425-434. [CrossRef]

273. Shirvani, G.; Fasihi-Harandi, M.; Raiesi, O.; Bazargan, N.; Zahedi, M.J.; Sharifi, I.; Kalantari-Khandani, B.; Nooshadokht, M.; Shabandoust, H.; Mohammadi, M.A.; et al. Prevalence and Molecular Subtyping of Blastocystis from Patients with Irritable Bowel Syndrome, Inflammatory Bowel Disease and Chronic Urticaria in Iran. Acta Parasitol. 2020, 65, 90-96. [CrossRef]

274. Barati, M.; Taghipour, A.; Bakhshi, B.; Shams, S.; Pirestani, M. Prevalence of Intestinal Parasitic Infections and Campylobacter spp. among Children with Gastrointestinal Disorders in Tehran, Iran. Parasite Epidemiol. Control 2021, 13. [CrossRef]

275. Bairami Kuzehkanani, A.; Rezaei, S.; Babaei, Z.; Niyyati, M.; Hashemi, S.N.; Rezaeian, M. Enteric Protozoan Parasites in Rural Areas of Bandar-Abbas, Southern Iran: Comparison of Past and Present Situation. Iran. J. Public Health 2011, 40, 80-85.

276. Sarkari, B.; Hosseini, G.; Motazedian, M.H.; Fararouei, M.; Moshfe, A. Prevalence and Risk Factors of Intestinal Protozoan Infections: A Population-Based Study in Rural Areas of Boyer-Ahmad District, Southwestern Iran. BMC Infect. Dis. 2016, 16, 703-710. [CrossRef]

277. Sharifdini, M.; Ghanbarzadeh, L.; Barikani, A.; Saraei, M. Prevalence of Intestinal Parasites among Rural Inhabitants of Fouman, Guilan Province, Northern Iran with Emphasis on Strongyloides stercoralis. Iran. J. Parasitol. 2020, 15, 91-100. [CrossRef] [PubMed]

278. Pagheh, A.S.; Sharif, M.; Daryani, A.; Yazdani-Charati, J.; Nazar, E.; Asfaram, S.; Hosseini, S.-A.; Tork, M.; Soosaraie, M.; Syadatpanah, A.; et al. A Cross-Sectional Analysis of Intestinal Parasitic Infections among the General Population in North of Iran. J. Infect. Dev. Ctries. 2018, 12, 120-126. [CrossRef] [PubMed]

279. Beiromvand, M.; Panabad, E.; Rafiei, A. Status of Intestinal Parasitic Infections among Rural and Urban Populations, Southwestern Iran. Asian Pac. J. Trop. Med. 2019, 12, 130-136. [CrossRef]

280. Taherkhani, K.; Barikani, A.; Shahnazi, M.; Saraei, M. Prevalence of Intestinal Parasites among Rural Residents of Takestan in North-West of Iran. Iran. J. Parasitol. 2019, 14, 657-663. [CrossRef]

281. Kim, M.-J.; Won, E.J.; Kim, S.H.; Shin, J.H.; Chai, J.-Y. Molecular Detection and Subtyping of Human Blastocystis and the Clinical Implications: Comparisons between Diarrheal and Non-Diarrheal Groups in Korean Populations. Korean J. Parasitol. 2020, 58, 321-326. [CrossRef]

282. Sayasone, S.; Mak, T.K.; Vanmany, M.; Rasphone, O.; Vounatsou, P.; Utzinger, J.; Akkhavong, K.; Odermatt, P. Helminth and Intestinal Protozoa Infections, Multiparasitism and Risk Factors in Champasack Province, Lao People's Democratic Republic. PLoS Negl. Trop. Dis. 2011, 5, e1037. [CrossRef]

283. Ribas, A.; Jollivet, C.; Morand, S.; Thongmalayvong, B.; Somphavong, S.; Siew, C.-C.; Ting, P.-J.; Suputtamongkol, S.; Saensombath, V.; Sanguankiat, S.; et al. Intestinal Parasitic Infections and Environmental Water Contamination in a Rural Village of Northern Lao PDR. Korean J. Parasitol. 2017, 55, 523-532. [CrossRef] [PubMed]

284. Sanpool, O.; Laymanivong, S.; Thanchomnang, T.; Rodpai, R.; Sadaow, L.; Phosuk, I.; Maleewong, W.; Intapan, P.M. Subtype Identification of Human Blastocystis spp. Isolated from Lao People's Democratic Republic. Acta Trop. 2017, 168, 37-40. [CrossRef] [PubMed]

285. Araj, G.F.; Musharraheh, U.M.; Haydar, A.; Ghawi, A.; Itani, R.; Saliba, R. Trends and Prevalence of Intestinal Parasites at a Tertiary Care Center in Lebanon over a Decade. J. Med. Liban. 2011, 59, 143-148.

286. Khaled, S.; Gantois, N.; Ayoubi, A.; Even, G.; Sawant, M.; El Houmayraa, J.; Nabot, M.; Benamrouz-Vanneste, S.; Chabé, M.; Certad, G.; et al. Blastocystis sp. Prevalence and Subtypes Distribution amongst Syrian Refugee Communities Living in North Lebanon. Microorganisms 2021, 9, 184. [CrossRef]

287. Sinniah, B.; Sabaridah, I.; Soe, M.M.; Sabitha, P.; Awang, I.P.R.; Ong, G.P.; Hassan, A.K.R. Determining the Prevalence of Intestinal Parasites in Three Orang Asli (Aborigines) Communities in Perak, Malaysia. Trop. Biomed. 2012, 29, $200-206$.

288. Mohammad, N.A.; Al-Mekhlafi, H.M.; Anuar, T.S. Subtype Distribution of Blastocystis Isolated from Humans and Associated Animals in an Indigenous Community with Poor Hygiene in Peninsular Malaysia. Trop. Biomed. 2018, 35, 849-860.

289. Noradilah, S.A.; Moktar, N.; Anuar, T.S.; Lee, I.L.; Salleh, F.M.; Manap, S.N.A.A.; Mohtar, N.S.H.M.; Azrul, S.M.; Abdullah, W.O.; Nordin, A.; et al. Microscopy-Based Techniques: A Reliable Method for Detecting Blastocystis sp. Infection. Sylwan 2017, 161, 149-153.

290. Mohammad, N.A.; Al-Mekhlafi, H.M.; Moktar, N.; Anuar, T.S. Prevalence and Risk Factors of Blastocystis Infection among Underprivileged Communities in Rural Malaysia. Asian Pac. J. Trop. Med. 2017, 10, 491-497. [CrossRef] [PubMed] 
291. Noradilah, S.A.; Moktar, N.; Anuar, T.S.; Lee, I.L.; Salleh, F.M.; Manap, S.N.A.A.; Mohtar, N.S.H.M.; Azrul, S.M.; Abdullah, W.O.; Nordin, A.; et al. Molecular Epidemiology of Blastocystosis in Malaysia: Does Seasonal Variation Play an Important Role in Determining the Distribution and Risk Factors of Blastocystis Subtype Infections in the Aboriginal Community? Parasites Vectors 2017, 10, 1-12. [CrossRef]

292. Noradilah, S.A.; Moktar, N.; Lee, I.L.; Salleh, F.M.; Anuar, T.S. Comparison of Direct Examination and in Vitro Culture for the Detection of Blastocystis sp. In Orang Asli Stool Samples. Sains Malays. 2018, 47, 2099-2104. [CrossRef]

293. Mohammad, N.A.; Al-Mekhlafi, H.M.; Anuar, T.S. Genetic Diversity of Blastocystis Isolates from Symptomatic and Asymptomatic Orang Asli in Pahang, Malaysia. Southeast Asian J. Trop. Med. Public Health 2018, 49, 189-197.

294. Muslim, A.; Sofian, S.M.; Shaari, S.A.; Hoh, B.-P.; Lim, Y.A.-L. Prevalence, Intensity and Associated Risk Factors of Soil Transmitted Helminth Infections: A Comparison between Negritos (Indigenous) in Inland Jungle and Those in Resettlement at Town Peripheries. PLoS Negl. Trop. Dis. 2019, 13, e0007331. [CrossRef]

295. Lee, I.L.; Tan, T.C.; Tan, P.C.; Nanthiney, D.R.; Biraj, M.K.; Surendra, K.M.; Suresh, K.G. Predominance of Blastocystis sp. Subtype 4 in Rural Communities, Nepal. Parasitol. Res. 2012, 110, 1553-1562. [CrossRef] [PubMed]

296. Santos, H.J.; Rivera, W.L. Comparison of Direct Fecal Smear Microscopy, Culture, and Polymerase Chain Reaction for the Detection of Blastocystis sp. in Human Stool Samples. Asian Pac. J. Trop. Med. 2013, 6, 780-784. [CrossRef]

297. Belleza, M.L.B.; Reyes, J.C.B.; Tongol-Rivera, P.N.; Rivera, W.L. Subtype Analysis of Blastocystis sp. Isolates from Human and Canine Hosts in an Urban Community in the Philippines. Parasitol. Int. 2016, 65, 291-294. [CrossRef]

298. Adao, D.E.; Dela Serna, A.O.; Belleza, M.L.; Bolo, N.R.; Rivera, W.L. Subtype Analysis of Blastocystis sp. Isolates from Asymptomatic Individuals in an Urban Community in the Philippines. Ann. Parasitol. 2016, 62, 193-200. [CrossRef] [PubMed]

299. Belleza, M.L.B.; Cadacio, J.L.C.; Borja, M.P.; Solon, J.A.A.; Padilla, M.A.; Tongol-Rivera, P.N.; Rivera, W.L. Epidemiologic Study of Blastocystis Infection in an Urban Community in the Philippines. J. Environ. Public Health 2015, 2015, 894297. [CrossRef]

300. Weerakoon, K.G.; Gordon, C.A.; Williams, G.M.; Cai, P.; Gobert, G.N.; Olveda, R.M.; Ross, A.G.; Olveda, D.U.; McManus, D.P. Co-Parasitism of Intestinal Protozoa and Schistosoma Japonicum in a Rural Community in the Philippines. Infect. Dis. Poverty 2018, 7, 121. [CrossRef]

301. AlDahhasi, W.; Toulah, F.; Wakid, M. Evaluation of Common Microspcpic Technique for Detection of Blastocystis hominis. J. Egypt. Soc. Parasitol. 2020, 50, 33-40. [CrossRef]

302. Hawash, Y.A.; Dorgham, L.S.; Amir, E.-A.M.; Sharaf, O.F. Prevalence of Intestinal Protozoa among Saudi Patients with Chronic Renal Failure: A Case-Control Study. J. Trop. Med. 2015, 2015, 563478. [CrossRef] [PubMed]

303. Alqumber, M.A. Association between Papio Hamadryas Populations and Human Gastrointestinal Infectious Diseases in Southwestern Saudi Arabia. Ann. Saudi Med. 2014, 34, 297-301. [CrossRef] [PubMed]

304. Kaewpitoon, N.; Kaewpitoon, S.; Meererksom, T.; Chan-Aran, S.; Sangwalee, W.; Norkaew, J.; Chuatanam, J.; Kujapan, J.; Padchasuwan, N.; Tongtawee, T. Detection of Opisthorchis Viverrini Infection among the ASEAN Population in Thailand Using a Verbal Screening Test and Fecal Concentrator Kit. Iran. J. Parasitol. 2018, 13, 258-266. [PubMed]

305. Kaewjai, C.; Prommano, O.; Tonsomboon, A. Detection of Blastocystis hominis Using Simple Direct Smear Compared with Jones' Medium Cultivation in Thatu Subdistrict, Chiang Khan Distric, Loei Province. Thammasat Med. J. 2020, $20,8-14$.

306. Popruk, N.; Prasongwattana, S.; Mahittikorn, A.; Palasuwan, A.; Popruk, S.; Palasuwan, D. Prevalence and Subtype Distribution of Blastocystis Infection in Patients with Diabetes Mellitus in Thailand. Int. J. Environ. Res. Public Health 2020, 17, 8877. [CrossRef] [PubMed]

307. Prommi, A.; Prombutara, P.; Watthanakulpanich, D.; Adisakwattana, P.; Kusolsuk, T.; Yoonuan, T.; Poodeepiyasawat, A.; Homsuwan, N.; Prummongkol, S.; Tanita, M.; et al. Intestinal Parasites in Rural Communities in Nan Province, Thailand: Changes in Bacterial Gut Microbiota Associated with Minute Intestinal Fluke Infection. Parasitology 2020, 147, 972-984. [CrossRef]

308. Yowang, A.; Tsaousis, A.D.; Chumphonsuk, T.; Thongsin, N.; Kullawong, N.; Popluechai, S.; Gentekaki, E. High Diversity of Blastocystis Subtypes Isolated from Asymptomatic Adults Living in Chiang Rai, Thailand. Infect. Genet. Evol. 2018, 65, 270-275. [CrossRef]

309. Punsawad, C.; Phasuk, N.; Bunratsami, S.; Thongtup, K.; Siripakonuaong, N.; Nongnaul, S. Prevalence of Intestinal Parasitic Infection and Associated Risk Factors among Village Health Volunteers in Rural Communities of Southern Thailand. BMC Public Health 2017, 17, 564. [CrossRef] [PubMed]

310. Palasuwan, A.; Palasuwan, D.; Mahittikorn, A.; Chiabchalard, R.; Combes, V.; Popruk, S. Subtype Distribution of Blastocystis in Communities along the Chao Phraya River, Thailand. Korean J. Parasitol. 2016, 54, 455-460. [CrossRef]

311. Kitvatanachai, S.; Taylor, A.; Rhongbutsri, P.; Taylor, W.R.J. Modified Harada-Mori and Simple Wet Mount to Determine Hookworm Infections in Yo Island Urban Area, Songkhla, Southern Thailand. Trop. Med. Health 2019, 47, 27. [CrossRef]

312. Boonjaraspinyo, S.; Boonmars, T.; Kaewsamut, B.; Ekobol, N.; Laummaunwai, P.; Aukkanimart, R.; Wonkchalee, N.; Juasook, A.; Sriraj, P. A Cross-Sectional Study on Intestinal Parasitic Infections in Rural Communities, Northeast Thailand. Korean J. Parasitol. 2013, 51, 727-734. [CrossRef]

313. Suntaravitun, P.; Dokmaikaw, A. Prevalence of Intestinal Parasites and Associated Risk Factors for Infection among Rural Communities of Chachoengsao Province, Thailand. Korean J. Parasitol. 2018, 56, 33-39. [CrossRef] [PubMed]

314. Wongthamarin, K.; Trairattanapa, T.; Kijanukul, S.; Kritsilpe, T.; Poobunjirdkul, S.; Chuengdee, W.; Mungthi, M.; Leelayoova, S.; Naaglor, T.; Taamasri, P.; et al. Blastocystis Incidence, Spontaneous Clearance, Persistence and Risk Factors in a Rural Community in Thailand: A Prospective Cohort Study. Asian Pac. J. Trop. Med. 2020, 13, 123-130. [CrossRef]

315. Popruk, S.; Udonsom, R.; Koompapong, K.; Mahittikorn, A.; Kusolsuk, T.; Ruangsittichai, J.; Palasuwan, A. Subtype Distribution of Blastocystis in Thai-Myanmar Border, Thailand. Korean J. Parasitol. 2015, 53, 13-19. [CrossRef] 
316. Karasartova, D.; Gureser, A.S.; Zorlu, M.; Turegun-Atasoy, B.; Taylan-Ozkan, A.; Dolapci, M. Blastocystosis in Post-Traumatic Splenectomized Patients. Parasitol. Int. 2016, 65, 802-805. [CrossRef] [PubMed]

317. Karadag, G.; Tamer, G.S.; Dervisoglu, E. Investigation of Intestinal Parasites in Dialysis Patients. Saudi Med. J. 2013, 34, 714-718. [PubMed]

318. Dogruman-Al, F.; Simsek, Z.; Boorom, K.; Ekici, E.; Sahin, M.; Tuncer, C.; Kustimur, S.; Altinbas, A. Comparison of Methods for Detection of Blastocystis Infection in Routinely Submitted Stool Samples, and Also in IBS/IBD Patients in Ankara, Turkey. PLoS ONE 2010, 5, e15484. [CrossRef]

319. Köksal, F.; Başlanti, I.; Samasti, M. A Retrospective Evaluation of the Prevalence of Intestinal Parasites in Istanbul, Turkey İstanbul'da Bağirsak Parazitlerinin Sikliği Bakimindan Retrospektif Bir Değerlendirme. Turk. Parazitolojii Derg. 2010, 34, 166-171.

320. Çetinkaya, Ü.; Yazar, S.; Kuk, S.; Ateş, S.; Hamamci, B.; Gedikbaş, T.; Şahin, İ. The Distribution of Intestinal Parasites Determined between 2009-2010 in Erciyes University, Medical Faculty, Parasitology Laboratory Erciyes Üniversitesi Tip Fakültesi Tibbi Parazitoloji Anabilim Dalı Laboratuvarında 2009-2010 Yılları Arasında Saptanan. Kafkas Univ. Vet. Fak. Derg. 2012, 18, A93-A96. [CrossRef]

321. Düzyol, D.; Kilimcioğlu, A.A.; Ozyurt, B.C.; Ozkan, H.; Girginkardeşler, N. Incidence of Intestinal Parasites Detected in the Department of Parasitology in Celal Bayar University Hospital between 2006 and 2010. Celal Bayar Üniversitesi Hastanesi Parazitoloji Polikliniği'nde 2006-2010 Yillari Arasinda Saptanan Bağirsak Parazit. Turk. Parazitolojii Derg. 2012, 36, 147-151. [CrossRef]

322. Kurt, Ö.; Akyar, I.; Görgün, S.; Kocagöz, T.; Özbilgin, A. Feconomics ${ }^{\circledR}$ : A Simple, Novel and Fast Technique for Stool Concentration in Parasitology Laboratory. Kafkas Univ. Vet. Fak. Derg. 2012, 18, A161-A165. [CrossRef]

323. Yilmaz, H.; Taş-Cengiz, Z.; Ceylan, A.; Ekici, A. The Distribution of Intestinal Parasites in People Admitted to the Yüzüncü Yil University Parasitology Laboratory of Health Research and Training Hospital, in 2009 Yüzüncü Yil Üniversitesi Araştirma ve Uygulama Hastanesi Parazitoloji Laboratuarina 20. Turk. Parazitolojii Derg. 2012, 36, 105-108. [CrossRef] [PubMed]

324. Gülmez, D.; Saribaş, Z.; Akyön, Y.; Ergüven, S. The Results of Hacettepe University Faculty of Medicine Parasitology Laboratory in 2003-2012: Evaluation of 10 Years Hacettepe Üniversitesi Tip Fakültesi Parazitoloji Laboratuvari 2003-2012 Yillari Sonuçlari: 10 Yillik Değerlendirme. Turk. Parazitolojii Derg. 2013, 37, 97-101. [CrossRef]

325. Kirkoyun Uysal, H.; Akgül, O.; Purisa, S.; Oner, Y.A. Twenty-Five Years of Intestinal Parasite Prevalence in İstanbul University, İstanbul Faculty of Medicine: A Retrospective Study İstanbul Üniversitesi İstanbul Tip Fakültesi'nde 25 Yillik İntestinal Parazit Prevalansi: Retrospektif Bir Çalişma. Turk. Parazitolojii Derg. 2014, 38, 97-101. [CrossRef] [PubMed]

326. Öncel, K. Distribution of Intestinal Parasites Detected in Şanlıurfa Mehmet Akif Inan Education and Research Hospital Between October 2015 and October 2016. Turk. Parazitolojii Derg. 2018, 42, 20-27. [CrossRef] [PubMed]

327. Malatyali, E.; Ertabaklar, H.; Ertuğ, S. Subtype Distribution of Blastocystis spp. With DNA Barcoding and Evaluation of Diagnostic Methods I DNA Barkotlama Yöntemiyle Blastocystis Alt Tiplerinin Belirlenmesi ve Tanı Yöntemlerinin Değerlendirilmesi. Mikrobiyol. Bul. 2019, 53, 308-318. [CrossRef]

328. Taş Cengiz, Z.; Yılmaz, H.; Beyhan, Y.E.; Çiçek, M. A Comprehensive Retrospective Study: Intestinal Parasites in Human in Van Province. Turk. Parazitolojii Derg. 2019, 43, 70-73. [CrossRef] [PubMed]

329. Gulhan, B.; Aydin, M.; Demirkazik, M.; Koltas, I.S.; Cikman, A.; Turkmen, K.; Duran, T. Subtype Distribution and Molecular Characterization of Blastocystis from Hemodialysis Patients in Turkey. J. Infect. Dev. Ctries. 2020, 14, 1448-1454. [CrossRef]

330. Koltas, I.S.; Eroglu, F. Subtype Analysis of Blastocystis Isolates Using SSU RRNA-DNA Sequencing in Rural and Urban Population in Southern Turkey. Exp. Parasitol. 2016, 170, 247-251. [CrossRef]

331. AbuOdeh, R.; Ezzedine, S.; Samie, A.; Stensvold, C.R.; ElBakri, A. Prevalence and Subtype Distribution of Blastocystis in Healthy Individuals in Sharjah, United Arab Emirates. Infect. Genet. Evol. 2016, 37, 158-162. [CrossRef]

332. Toychiev, A.; Navruzov, B.; Pazylova, D.; Davis, N.; Badalova, N.; Osipova, S. Intestinal Protozoa and Helminths in Ulcerative Colitis and the Influence of Anti-Parasitic Therapy on the Course of the Disease. Acta Trop. 2021, 213, 105755. [CrossRef]

333. Hatipoğlu, S.; Lök, U.; Gülaçtı, U.; Çelik, T. Pre-Operative Stool Analysis for Intestinal Parasites and Fecal Occult Blood in Patients with Acute Appendicitis Akut Apandisit Hastalarinda Gaitada Gizli Kan ve Intestinal Parazitler Için Ameliyat Öncesi Gaita Analizi. Turk. J. Trauma Emerg. Surg. 2016, 22, 471-476. [CrossRef]

334. Angal, L.; Mahmud, R.; Samin, S.; Yap, N.-J.; Ngui, R.; Amir, A.; Ithoi, I.; Kamarulzaman, A.; AL Lim, Y. Determining Intestinal Parasitic Infections (IPIs) in Inmates from Kajang Prison, Selangor, Malaysia for Improved Prison Management. BMC Infect. Dis. 2015, 15, 467. [CrossRef]

335. Chandramathi, S.; Suresh, K.; Kuppusamy, U.R. Elevated Levels of Urinary Hyaluronidase in Humans Infected with Intestinal Parasites. Ann. Trop. Med. Parasitol. 2010, 104, 449-452. [CrossRef] [PubMed]

336. Rezaei Riabi, T.; Mirjalali, H.; Haghighi, A.; Rostami Nejad, M.; Pourhoseingholi, M.A.; Poirier, P.; Delbac, F.; Wawrzyniak, I.; Zali, M.R. Genetic Diversity Analysis of Blastocystis Subtypes from Both Symptomatic and Asymptomatic Subjects Using a Barcoding Region from the 18S RRNA Gene. Infect. Genet. Evol. 2018, 61, 119-126. [CrossRef]

337. Vezir, S.; Kaya, F.; Vezir, E.; Karaosmanoğlu, N.; Adiloğlu, A.K. Evaluation of Intestinal Parasites in Patients with Chronic Spontaneous Urticaria in a Territory Hospital in Turkey. J. Infect. Dev. Ctries. 2019, 13, 927-932. [CrossRef] [PubMed]

338. Aydin, M.; Yazici, M.; Demirkazik, M.; Koltas, I.; Cikman, A.; Gulhan, B.; Duran, T.; Yilmaz, A.; Kara, M. Molecular Characterization and Subtyping of Blastocystis in Urticarial Patients in Turkey. Asian Pac. J. Trop. Med. 2019, 12, 450-456. [CrossRef]

339. Yildiz, S.; Dogan, I.; Dogruman-Al, F.; Nalbantoglu, U.; Ustek, D.; Sarzhanov, F.; Yildirim, S. Association of Enteric Protist Blastocystis spp. and Gut Microbiota with Hepatic Encephalopathy. J. Gastrointest. Liver Dis. 2016, 25, 489-497. [CrossRef] [PubMed] 
340. Alinaghizade, A.; Mirjalali, H.; Mohebali, M.; Stensvold, C.R.; Rezaeian, M. Inter- and Intra-Subtype Variation of Blastocystis Subtypes Isolated from Diarrheic and Non-Diarrheic Patients in Iran. Infect. Genet. Evol. 2017, 50, 77-82. [CrossRef]

341. Thergarajan, G.; Kumar, S.; Bhassu, S.; Omar, S.F.B.S.; Rampal, S. Effect of Blastocystis sp. In Dengue Patients-Increase in the Treatment Cost and Exacerbation of Symptoms. PLoS ONE 2019, 14, e0211034. [CrossRef]

342. Roy, M.; Singha, B.; Dhar, D.; Roychoudhury, S. Prevalence of Giardia Intestinalis with Other Co-Infecting Parasites in Barak Valley, Assam, India: A Molecular Approach. J. Parasit. Dis. 2019, 43, 426-442. [CrossRef]

343. Barazesh, A.; Fouladvand, M.; Tahmasebi, R.; Heydari, A.; Fallahi, J. The Prevalence of Intestinal Parasites in Hemodialysis Patients in Bushehr, Iran. Hemodial. Int. 2015, 19, 447-451. [CrossRef]

344. Pipatsatitpong, D.; Rangsin, R.; Leelayoova, S.; Naaglor, T.; Mungthin, M. Incidence and Risk Factors of Blastocystis Infection in an Orphanage in Bangkok, Thailand. Parasites Vectors 2012, 5, 37. [CrossRef]

345. Koltas, I.S.; Akyar, I.; Elgun, G.; Kocagoz, T. Feconomics; a New and More Convenient Method, the Routine Diagnosis of Intestinal Parasiticinfections. Parasitol. Res. 2014, 113, 2503-2508. [CrossRef] [PubMed]

346. Ozbagcivan, O.; Akarsu, S.; Avci, C.; Inci, B.B.; Fetil, E. Examination of the Microbial Spectrum in the Etiology of Erythema Nodosum: A Retrospective Descriptive Study. J. Immunol. Res. 2017, 2017, 8139591. [CrossRef]

347. Tunalı, V.; Öztürk, E.A.; Ünver, A.; Turgay, N. The Prevalance of Blastocystosis among Patients with Gastrointestinal and Dermatologic Complaints and Effects of Blastocystis spp. Density on Symptomatology. Turk. Parazitolojii Derg. 2018, 42, $254-257$. [CrossRef] [PubMed]

348. Jafari, R.; Gorgizadeh, H.; Soosaraei, M.; Shokri, A.; Bandalizadeh, Z.; Ahmadi, H.-Y.; Banimostafavi, E.S.; Fakhar, M. Initial Evidences of Salt and Pepper Retinal Lesions (Sprl) in Patients with Intestinal Protozoan Infections in Iran. Infect. Disord. Drug Targets 2021, 21, 60-67. [CrossRef]

349. Teh, C.L.; Wan, S.A.; Ling, G.R. Severe Infections in Systemic Lupus Erythematosus: Disease Pattern and Predictors of InfectionRelated Mortality. Clin. Rheumatol. 2018, 37, 2081-2086. [CrossRef] [PubMed]

350. Malatyalı, E.; Sankur, F.; Akın, M.N.; Ertabaklar, H.; Ertuğ, S. Subtype Distribution of Blastocystis in Pregnant Women and Analysis of Possible Risk Factors. Turk. Parazitolojii Derg. 2020, 44, 221-225. [CrossRef]

351. Tai, W.-P.; Hu, P.-J.; Wu, J.; Lin, X.-C. Six Ulcerative Colitis Patients with Refractory Symptoms Co-Infective with Blastocystis Hominis in China. Parasitol. Res. 2011, 108, 1207-1210. [CrossRef] [PubMed]

352. Zhao, G.H.; Hu, X.F.; Liu, T.L.; Hu, R.S.; Yu, Z.Q.; Yang, W.B.; Wu, Y.L.; Yu, S.K.; Song, J.K. Molecular Characterization of Blastocystis sp. in Captive Wild Animals in Qinling Mountains. Parasitol. Res. 2017, 116, 2327-2333. [CrossRef]

353. Li, X.-D.; Zou, Y.; Pan, J.; Liang, Q.-L.; Zeng, Z.; Meng, Y.-M.; Wang, X.-L.; Wang, H.-N.; Zhu, X.-Q. Prevalence and Subtypes of Blastocystis Sp. Infection in Zoo Animals in Three Cities in China. Parasitol. Res. 2020, 119, 465-471. [CrossRef]

354. Ma, Y.T.; Liu, Q.; Xie, S.C.; Li, X.D.; Ma, Y.Y.; Li, T.S.; Gao, W.W.; Zhu, X.Q. Prevalence and Subtypes of Blastocystis in Alpacas, Vicugna Pacos in Shanxi Province, China. Korean J. Parasitol. 2020, 58, 181-184. [CrossRef] [PubMed]

355. Sreekumar, C.; Selvaraj, J.; Gomathinayagam, S.; Thangapandiyan, M.; Ravikumar, G.; Roy, P.; Balachandran, C. Blastocystis sp. from Food Animals in India. J. Parasit. Dis. 2014, 38, 440-443. [CrossRef] [PubMed]

356. Hemalatha, C.; Chandrawathani, P.; Suresh Kumar, G.; Premaalatha, B.; Geethamalar, S.; Lily Rozita, M.; Farah Haziqah, M.T.; Sabapathy, D.; Ramlan, M. The Diagnosis of Blastocystis sp. From Animals an Emerging Zoonosis. Malays. J. Vet. Res. 2014, 5, 15-22.

357. Abd Razak, N.A.; Yusof, A.M.; Mohammad, M. Identification of Blastocystis sp. Infection from Cattle, Goat and Sheep Isolated from Farms in Pahang, Malaysia. Int. J. Allied Health Sci. 2019, 3, 810.

358. Kamaruddin, S.K.; Mat Yusof, A.; Mohammad, M. Prevalence and Subtype Distribution of Blastocystis sp. in Cattle from Pahang, Malaysia. Trop. Biomed. 2020, 37, 127-141.

359. Aynur, Z.E.; Güçlü, Ö.; Yıldız, İ; Aynur, H.; Ertabaklar, H.; Bozdoğan, B.; Ertuğ, S. Molecular Characterization of Blastocystis in Cattle in Turkey. Parasitol. Res. 2019, 118, 1055-1059. [CrossRef]

360. Hastutiek, P.; Yuniarti, W.M.; Djaeri, M.; Lastuti, N.D.R.; Suprihati, E.; Suwanti, L.T. Prevalence and Diversity of Gastrointestinal Protozoa in Madura Cattle at Bangkalan Regency, East Java, Indonesia. Vet. World 2019, 12, 198-204. [CrossRef]

361. Susana, Y.; Suwanti, L.T.; Suprihati, E. Identification and Prevalence of Gastrointestinal Parasites in Beef Cattle in Siak Sri Indrapura, Riau, Indonesia. Indones. J. Trop. Infect. Dis. 2019, 7, 155-160. [CrossRef]

362. Suwanti, L.T.; Susana, Y.; Hastutiek, P.; Suprihati, E.; Lastuti, N.D.R. Blastocystis spp. Subtype 10 Infected Beef Cattle in Kamal and Socah, Bangkalan, Madura, Indonesia. Vet. World 2020, 13, 231-237. [CrossRef] [PubMed]

363. Badparva, E.; Sadraee, J.; Kheirandish, F. Genetic Diversity of Blastocystis Isolated from Cattle in Khorramabad, Iran. Jundishapur J. Microbiol. 2015, 8, 4-7. [CrossRef] [PubMed]

364. Rostami, M.; Fasihi-Harandi, M.; Shafiei, R.; Aspatwar, A.; Derakhshan, F.K.; Raeghi, S. Genetic Diversity Analysis of Blastocystis Subtypes and Their Distribution among the Domestic Animals and Pigeons in Northwest of Iran. Infect. Genet. Evol. 2020, 86, 104591. [CrossRef] [PubMed]

365. Masuda, A.; Sumiyoshi, T.; Ohtaki, T.; Matsumoto, J. Prevalence and Molecular Subtyping of Blastocystis from Dairy Cattle in Kanagawa, Japan. Parasitol. Int. 2018, 67, 702-705. [CrossRef] [PubMed]

366. Zhu, W.; Tao, W.; Gong, B.; Yang, H.; Li, Y.; Song, M.; Lu, Y.; Li, W. First Report of Blastocystis Infections in Cattle in China. Vet. Parasitol. 2017, 246, 38-42. [CrossRef]

367. Wang, J.; Gong, B.; Yang, F.; Zhang, W.; Zheng, Y.; Liu, A. Subtype Distribution and Genetic Characterizations of Blastocystis in Pigs, Cattle, Sheep and Goats in Northeastern China's Heilongjiang Province. Infect. Genet. Evol. 2018, 57, 171-176. [CrossRef] 
368. Lee, H.; Lee, S.-H.; Seo, M.-G.; Kim, H.-Y.; Kim, J.W.; Lee, Y.-R.; Kim, J.H.; Kwon, O.-D.; Kwak, D. Occurrence and Genetic Diversity of Blastocystis in Korean Cattle. Vet. Parasitol. 2018, 258, 70-73. [CrossRef]

369. AbuOdeh, R.; Ezzedine, S.; Madkour, M.; Stensvold, C.R.; Samie, A.; Nasrallah, G.; AlAbsi, E.; ElBakri, A. Molecular Subtyping of Blastocystis from Diverse Animals in the United Arab Emirates. Protist 2019, 170, 125679. [CrossRef]

370. Mirzapour, A.; Kiani, H.; Mobedi, I.; Spotin, A.; Seyyed Tabaei, S.J.; Rahimi, M. Frequency of Intestinal Parasites among Zoo Animal by Morphometric Criteria and First Report of the Bivitellobilharzia Nairi from Elephant (Elephasmaximus Maximus) in Iran. Iran. J. Parasitol. 2018, 13, 611-617.

371. Mohammad, N.A.; Al-Mekhlafi, H.M.; Moktar, N.; Anuar, T.S. Molecular Detection and Subtyping of Blastocystis in Javan Rusa (Cervus timorensis) and Sika Deer (Cervus nippon) from Peninsular Malaysia. Thai J. Vet. Med. 2018, 48, $295-301$.

372. Mohd Zain, S.N.; Farah Haziqah, M.T.; Woh, P.Y.; Fazly Ann, Z.; Vickneshwaran, M.; Mohd Khalid, M.K.N.; Arutchelvan, R.; Suresh, K. Morphological and Molecular Detection of Blastocystis in Wildlife from Tioman Island, Malaysia. Trop. Biomed. 2017, 34, 249-255. [PubMed]

373. Wang, J.; Gong, B.; Liu, X.; Zhao, W.; Bu, T.; Zhang, W.; Liu, A.; Yang, F. Distribution and Genetic Diversity of Blastocystis Subtypes in Various Mammal and Bird Species in Northeastern China. Parasites Vectors 2018, 11, 522. [CrossRef]

374. Ni, H.-B.; Gong, Q.-L.; Zhang, N.-Z.; Zhao, Q.; Tao, W.-F.; Qiu, H.-Y.; Fei, Y.-C.; Zhang, X.-X. Molecular Detection of Blastocystis in Black Bears and Sika Deer in Northern China. Parasitol. Res. 2021, 120, 1481-1487. [CrossRef]

375. Li, J.; Karim, M.R.; Li, D.; Rahaman Sumon, S.M.M.; Siddiki, S.H.M.F.; Rume, F.I.; Sun, R.; Jia, Y.; Zhang, L. Molecular Characterization of Blastocystis sp. in Captive Wildlife in Bangladesh National Zoo: Non-Human Primates with High Prevalence and Zoonotic Significance. Int. J. Parasitol. Parasites Wildl. 2019, 10, 314-320. [CrossRef]

376. Kim, K.T.; Noh, G.; Lee, H.; Kim, S.H.; Jeong, H.; Kim, Y.; Jheong, W.H.; Oem, J.K.; Kim, T.H.; Kwon, O.D.; et al. Genetic Diversity and Zoonotic Potential of Blastocystis in Korean Water Deer, Hydropotes inermis argyropus. Pathogens 2020, 9, 955. [CrossRef]

377. Song, J.K.; Yin, Y.L.; Yuan, Y.J.; Tang, H.; Ren, G.J.; Zhang, H.J.; Li, Z.X.; Zhang, Y.M.; Zhao, G.H. First Genotyping of Blastocystis sp. in Dairy, Meat, and Cashmere Goats in Northwestern China. Acta Trop. 2017, 176, 277-282. [CrossRef] [PubMed]

378. Li, W.C.; Wang, K.; Gu, Y. Occurrence of Blastocystis sp. and Pentatrichomonas hominis in Sheep and Goats in China. Parasites Vectors 2018, 11, 93. [CrossRef]

379. Ghimire, T.R.; Bhattarai, N. A Survey of Gastrointestinal Parasites of Goats in a Goat Market in Kathmandu, Nepal. J. Parasit. Dis. 2019, 43, 686-695. [CrossRef] [PubMed]

380. Tan, T.C.; Tan, P.C.; Sharma, R.; Sugnaseelan, S.; Suresh, K.G. Genetic Diversity of Caprine Blastocystis from Peninsular Malaysia. Parasitol. Res. 2013, 112, 85-89. [CrossRef]

381. Adao, D.E.V.; Ducusin, R.J.T.; Padilla, M.A.; Rivera, W.L. Molecular Characterization of Blastocystis Isolates Infecting Farm Animals in Victoria and Pila, Laguna, Philippines. Philipp. Agric. Sci. 2016, 99, 304-310.

382. Adhikari, J.N.; Adhikari, R.B.; Bhattarai, B.P.; Thapa, T.B.; Ghimire, T.R. Short Communication: A Small-Scale Coprological Survey of the Endoparasites in the Himalayan Goral Naemorhedus goral (Hardwick, 1825) in Nepal. Biodiversitas 2021, 22, 1285-1290. [CrossRef]

383. Song, J.K.; Hu, R.S.; Fan, X.C.; Wang, S.S.; Zhang, H.J.; Zhao, G.H. Molecular Characterization of Blastocystis from Pigs in Shaanxi Province of China. Acta Trop. 2017, 173, 130-135. [CrossRef] [PubMed]

384. Wang, R.; Zhang, Y.; Jiang, Y.; Xing, J.; Tao, D.; Qi, M. First Report of Blastocystis Infection in Pigs from Large Farms in Xinjiang, China. J. Eukaryot. Microbiol. 2020, 67, 642-647. [CrossRef]

385. Han, J.Q.; Li, Z.; Zou, Y.; Pu, L.H.; Zhu, X.Q.; Zou, F.C.; Huang, C.Q. Prevalence, Molecular Characterization and Risk Factors of Blastocystis sp. from Farmed Pigs in Yunnan Province, Southwestern China. Acta Parasitol. 2020, 65, 1005-1010. [CrossRef]

386. Zou, Y.; Yang, W.B.; Zou, F.C.; Lin, R.Q.; Zhu, X.Q.; Hou, J.L. Molecular Detection and Subtype Distribution of Blastocystis in Farmed Pigs in Southern China. Microb. Pathog. 2021, 151, 104751. [CrossRef]

387. Arpitha, G.M.; Sreekumar, C.; Latha, B.R.; Vijaya Bharathi, M. Prevalence and Staining Characteristics of Blastocystis Isolates from Food Animals in Tamil Nadu. Vet. Parasitol. Reg. Stud. Rep. 2018, 11, 61-65. [CrossRef] [PubMed]

388. Mahendra, D.; Suwanti, L.T.; Lastuti, N.D.R.; Mufasirin; Suprihati, E.; Yuniarti, W.M.; Widisuputri, N.K.A. Deteksi Molekuler Blastocystis sp. Pada Babi Terinfeksi Di Kabupaten Tabanan Dan Badung, Provinsi Bali, Indonesia. J. Vet. 2020, 21, 227-233. [CrossRef]

389. Widisuputri, N.K.A.; Suwanti, L.T.; Plumeriastuti, H. A Survey for Zoonotic and Other Gastrointestinal Parasites in Pig in Bali Province, Indonesia. Indones. J. Trop. Infect. Dis. 2020, 8, 54-65. [CrossRef]

390. Paik, S.; Jung, B.Y.; Lee, H.; Hwang, M.H.; Han, J.E.; Rhee, M.H.; Kim, T.H.; Kwon, O.D.; Kwak, D. Molecular Detection and Subtyping of Blastocystis in Korean Pigs. Korean J. Parasitol. 2019, 57, 525-529. [CrossRef]

391. Adao, D.E.V.; Ronquillo, I.D.J.; Dela Cruz, Y.K.M.; Pagoso, E.J.A.; Rivera, W.L. Molecular Characterization of Giardia Duodenalis and Blastocystis sp. in Livestock from Animal Farms in Bulacan, Philippines. Southeast Asian J. Trop. Med. Public Health 2019, 50, 450-460.

392. De La Cruz, C.P.P.; Gorospe, M.M.; Paller, V.G.V. Blastocystis Infection among Backyard-Raised Pigs in Bay, Province of Laguna, the Philippines. Asian J. Microbiol. Biotechnol. Environ. Sci. 2016, 18, 487-494.

393. Evidor, F.M.R.; Rivera, W.L. Genetic Subtypes of Blastocystis sp. Isolated from Fecal Materials in the Large Intestines of Slaughtered Swine. Philipp. J. Vet. Med. 2016, 53, 59-64. 
394. Murao, L.A.E.; Cadiz, A.E.; De St, Y.; Ladera, L.; Montajes, K.P.; Catherine, M.; Pierre, B.O.; Gilles, G.; Oponda, N.B.; Lagat, F.T.; et al. Exploratory Investigation on the Occurrence, Spatial Distribution, and Risk Factors of Selected Zoonotic Enteropathogens in Davao City Backyard Farms. 2018. Available online: http:/ / ojs.upmin.edu.ph/index.php/banwa-b/article/ view/339 (accessed on 30 August 2021).

395. Sanyanusin, S.; Mori, H.; Prasertbun, R.; Pintong, A.; Komalamisra, C.; Changbunjong4, T.; Popruk, S.; Mahittikorn, A. Molecular Detection and Genotyping of Blastocystis and Enterocytozoon bieneusi in Humans and Pigs in Nakhon Pathom Province, Thailand. Jt. Int. Trop. Med. Meet. Proc. 2017, 6, 1-6.

396. Alfellani, M.A.; Taner-Mulla, D.; Jacob, A.S.; Imeede, C.A.; Yoshikawa, H.; Stensvold, C.R.; Clark, C.G. Genetic Diversity of Blastocystis in Livestock and Zoo Animals. Protist 2013, 164, 497-509. [CrossRef]

397. Lee, H.; Seo, M.-G.; Oem, J.-K.; Kim, Y.-S.; Lee, S.-Y.; Kim, J.; Jeong, H.; Jheong, W.-H.; Kim, Y.; Lee, W.-J.; et al. Molecular Detection and Subtyping of Blastocystis Detected in Wild Boars (Sus scrofa) in South Korea. J. Wildl. Dis. 2020, 56, 662-666. [CrossRef] [PubMed]

398. Yaghoobi, K.; Sarkari, B.; Mansouri, M.; Motazedian, M.H. Zoonotic Intestinal Protozoan of the Wild Boars, Sus scrofa, in Persian Gulf's Coastal Area (Bushehr Province), Southwestern Iran. Vet. World 2016, 9, 1047-1050. [CrossRef] [PubMed]

399. Ren, M.; Song, J.K.; Yang, F.; Zou, M.; Wang, P.X.; Wang, D.; Zhang, H.J.; Zhao, G.H.; Lin, Q. First Genotyping of Blastocystis in Yaks from Qinghai Province, Northwestern China. Parasites Vectors 2019, 12, 171. [CrossRef]

400. Li, W.; Liu, X.; Gu, Y.; Liu, J.; Luo, J. Prevalence of Cryptosporidium, Giardia, Blastocystis, and Trichomonads in Domestic Cats in East China. J. Vet. Med. Sci. 2019, 81, 890-896. [CrossRef] [PubMed]

401. Patagi, A.L.; Suwanti, L.T.; Chairul, A.; Yuniarti, W.M.; Sarmanu; Suprihati, E. Prevalance of Gastrointestinal Protozoa of Cats in Animal Hospital and Animal Clinic in Surabaya. J. Parasite Sci. 2018, 2, 61-66.

402. Khademvatan, S.; Abdizadeh, R.; Rahim, F.; Hashemitabar, M.; Ghasemi, M.; Tavalla, M. Stray Cats Gastrointestinal Parasites and Its Association with Public Health in Ahvaz City, South Western of Iran. Jundishapur J. Microbiol. 2014, 7. [CrossRef]

403. Mohammadpour, I.; Bozorg-Ghalati, F.; Gazzonis, A.L.; Manfredi, M.T.; Motazedian, M.H.; Mohammadpour, N. First Molecular Subtyping and Phylogeny of Blastocystis sp. Isolated from Domestic and Synanthropic Animals (Dogs, Cats and Brown Rats) in Southern Iran. Parasites Vectors 2020, 13, 365. [CrossRef]

404. Kwak, D.; Seo, M.-G. Genetic Analysis of Zoonotic Gastrointestinal Protozoa and Microsporidia in Shelter Cats in South Korea. Pathogens 2020, 9, 894. [CrossRef]

405. Farah Haziqah, M.T.; Chandrawathani, P.; Douadi, B.; Suresh, K.; Wilson, J.J.; Mohd Khalid, M.K.N.; Rajamanikam, A.; Lewis, J.W.; Mohd Zain, S.N. Impact of PH on the Viability and Morphology of Blastocystis Isolates. Trop. Biomed. 2018, 35, 501-510.

406. Mohammad Rahimi, H.; Nemati, S.; Mirjalali, H.; Sharifdini, M.; Zali, M.R. Molecular Characterization and Identification of Blastocystis and Its Subtypes from Raccoon (Procyon lotor) in North of Iran. Parasitol. Res. 2020, 119, 2741-2745. [CrossRef]

407. Liao, S.; Lin, X.; Sun, Y.; Qi, N.; Lv, M.; Wu, C.; Li, J.; Hu, J.; Yu, L.; Cai, H.; et al. Occurrence and Genotypes of Cryptosporidium spp., Giardia duodenalis, and Blastocystis sp. in Household, Shelter, Breeding, and Pet Market Dogs in Guangzhou, Southern China. Sci. Rep. 2020, 10, 17736. [CrossRef] [PubMed]

408. Wang, W.; Cuttell, L.; Bielefeldt-Ohmann, H.; Inpankaew, T.; Owen, H.; Traub, R.J. Diversity of Blastocystis Subtypes in Dogs in Different Geographical Settings. Parasites Vectors 2013, 6, 215-219. [CrossRef] [PubMed]

409. Mohaghegh, M.A.; Vafaei, M.R.; Ghomashlooyan, M.; Azami, M.; Falahati, M.; Azadi, Y.; Yousefi, H.A.; Jabalameli, Z.; Hejazi, S.H. A Wide Diversity of Zoonotic Intestinal Parasites in Domestic and Stray Dogs in Rural Areas of Kermanshah Province, Iran. Trop. Biomed. 2018, 35, 82-90.

410. Mirbadie, S.R.; Najafi Nasab, A.; Mohaghegh, M.A.; Norouzi, P.; Mirzaii, M.; Spotin, A. Molecular Phylodiagnosis of Echinococcus granulosus Sensu Lato and Taenia hydatigena Determined by Mitochondrial Cox1 and SSU-RDNA Markers in Iranian Dogs: Indicating the First Record of Pig Strain (G7) in Definitive Host in the Middle East. Comp. Immunol. Microbiol. Infect. Dis. 2019, 65, 88-95. [CrossRef]

411. Deng, L.; Yao, J.-X.; Liu, H.-F.; Zhou, Z.-Y.; Chai, Y.-J.; Wang, W.-Y.; Zhong, Z.-J.; Deng, J.-L.; Ren, Z.-H.; Fu, H.-L.; et al. First Report of Blastocystis in Giant Pandas, Red Pandas, and Various Bird Species in Sichuan Province, Southwestern China. Int. J. Parasitol. Parasites Wildl. 2019, 9, 298-304. [CrossRef]

412. Zanzani, S.A.; Gazzonis, A.L.; Epis, S.; Manfredi, M.T. Study of the Gastrointestinal Parasitic Fauna of Captive Non-Human Primates (Macaca fascicularis). Parasitol. Res. 2016, 115, 307-312. [CrossRef]

413. Li, T.C.; Li, Z.; Zhang, Y.L.; Chen, W.J.; Dong, X.L.; Yang, J.F.; Li, H.X.; Zou, F.C. Assessment of the Subtypes and the Zoonotic Risk of Blastocystis sp. of Experimental Macaques in Yunnan Province, Southwestern China. Parasitol. Res. 2020, 119, 741-748. [CrossRef] [PubMed]

414. Casim, L.F.; Bandal, M.Z.; Gonzales, J.C.B.; Valdez, E.M.M., Jr.; Chavez, G.C.S.; Paller, V.G.V. Enteroparasites of Captive LongTailed Macaques (Macaca fascicularis) from National Wildlife Research and Rescue Center, Diliman, Quezon City, Philippines. Asian J. Conserv. Biol. 2015, 4, 54-61.

415. Vaisusuk, K.; Saijuntha, W.; Sedlak, S.; Thanchomnang, T.; Pilap, W.; Suksavate, W.; Stensvold, C.R.; Tantrawatpan, C. Blastocystis Subtypes Detected in Long-Tailed Macaques in Thailand-Further Evidence of Cryptic Host Specificity. Acta Trop. 2018, 184, 78-82. [CrossRef]

416. Labes, E.M.; Hegglin, D.; Grimm, F.; Nurcahyo, W.; Harrison, M.E.; Bastian, M.L.; Deplazes, P. Intestinal Parasites of Endangered Orangutans (Pongo pygmaeus) in Central and East Kalimantan, Borneo, Indonesia. Parasitology 2010, 137, 123-135. [CrossRef] 
417. Dalimi, A.; Motamedi, G.; Hablolvarid, M.H.; Abdoli, A. Alimentary Tract Parasites of Vervet Monkeys (Cercopithecus aethiops): A Potential Reservoir for Human Transmission. Arch. Razi Inst. 2016, 71, 277-281. [CrossRef]

418. Ma, L.; Zhang, Y.; Qiao, H.; Li, S.; Wang, H.; Zhang, N.; Zhang, X. Cockroach as a Vector of Blastocystis sp. Is Risk for Golden Monkeys in Zoo. Korean J. Parasitol. 2020, 58, 583-587. [CrossRef] [PubMed]

419. Adrus, M.; Zainudin, R.; Ahamad, M.; Jayasilan, M.A.; Abdullah, M.T. Gastrointestinal Parasites of Zoonotic Importance Observed in the Wild, Urban, and Captive Populations of Non-Human Primates in Malaysia. J. Med. Primatol. 2019, 48, 22-31. [CrossRef] [PubMed]

420. Farah Haziqah, M.T.; Chandrawathani, P.; Mohd, Z.S.N.; Suresh, K.G.; Hemalatha, C.; Premaalatha, B. A Preliminary Study of Blastocystis sp. Isolated from Chicken in Perak and Selangor, Malaysia. Malays. J. Vet. Res. 2014, 5, 21-25.

421. Farah Haziqah, M.T.; Chandrawathani, P.; Suresh, K.G.; Wilson, J.-J.; Mohd Khairul Nizam, M.K.; Rajamanikam, A.; Bathmanaban, P.; Siti, N.M.Z. Prevalence, Ultrastructure and Subtypes of Blastocystis In Chickens (Gallus gallus) From Peninsular Malaysia. Southeast Asian J. Trop. Med. Public Health 2018, 49, 921-932.

422. Zhang, X.; Qiao, J.Y.; Wu, X.M.; Ma, Q.Y.; Hu, H.; Wang, J.; Che, L.F. Ascaris Spp. and Capillaria Caudinflata Infections in Captive-Bred Crested Ibis (Nipponia nippon) in China. Zoo Biol. 2014, 34, 80-84. [CrossRef]

423. Asghari, A.; Sadraei, J.; Pirestani, M.; Mohammadpour, I. First Molecular Identification and Subtype Distribution of Blastocystis sp. Isolated from Hooded Crows (Corvus cornix) and Pigeons (Columba livia) in Tehran Province, Iran. Comp. Immunol. Microbiol. Infect. Dis. 2019, 62, 25-30. [CrossRef]

424. Chandrasekaran, H.; Govind, S.K.; Panchadcharam, C.; Bathmanaban, P.; Raman, K.; Thergarajan, G. High Lipid Storage in Vacoular Forms of Subtype 6 Blastocystis sp. in Ostrich. Parasites Vectors 2014, 7, 1-7. [CrossRef]

425. Xiao, X.; Zhou, S.-H.; Jiang, N.; Tian, D.-Z.; Zhou, Z.-M.; Zhang, M.; Ke, H.; Jiang, X.-C.; Lv, W.-L.; Gao, Q.-H. First Record of Leptospira and Blastocystis Infections in Captive Flying Squirrels (Trogopterus xanthipes) from Enshi County, China. Acta Trop. 2019, 197, 105065. [CrossRef]

426. Chai, Y.; Deng, L.; Liu, H.; Yao, J.; Zhong, Z.; Fu, H.; Shen, L.; Zhou, Z.; Deng, J.; Hu, Y.; et al. First Subtyping of Blastocystis sp. from Pet Rodents in Southwestern China. Int. J. Parasitol. Parasites Wildl. 2020, 11, 143-148. [CrossRef] [PubMed]

427. Li, J.; Yuan, Y.; Jiang, Y.; Wang, W.; Chao, L.; Sun, R.; Li, J.; Karim, M.R.; Qi, M. Molecular Identification and Subtyping of Blastocystis sp. In Laboratory Rats in China. Parasite 2020, 27, 35. [CrossRef] [PubMed]

428. Prasetyo, R.H. Survey of House Rat Intestinal Parasites from Surabaya District, East Java, Indonesia That Can Cause Opportunistic Infections in Humans. Southeast Asian J. Trop. Med. Public Health 2016, 47, 194-198.

429. Katsumata, M.; Yoshikawa, H.; Tokoro, M.; Mizuno, T.; Nagamoto, T.; Hendarto, J.; Asih, P.B.S.; Rozi, I.E.; Kimata, I.; Takami, K.; et al. Molecular Phylogeny of Blastocystis Isolates from Wild Rodents Captured in Indonesia and Japan. Parasitol. Res. 2018, 117, 2841-2846. [CrossRef] [PubMed]

430. Seifollahi, Z.; Sarkari, B.; Motazedian, M.H.; Asgari, Q.; Ranjbar, M.J.; Abdolahi Khabisi, S. Protozoan Parasites of Rodents and Their Zoonotic Significance in Boyer-Ahmad District, Southwestern Iran. Vet. Med. Int. 2016, 2016, 3263868. [CrossRef] [PubMed]

431. Premaalatha, B.; Chandrawathani, P.; Priscilla, F.X.; Farah Haziqah, M.; Jamnah, O.; Zaini, C.M.; Ramlan, M. A Survey of Endoparasite and Ectoparasite Infections of Wild Rats Caught in Areas of Ipoh and Kuala Lumpur, Malaysia. Malays. J. Vet. Res. 2017, 8, 29-34.

432. Farah Haziqah, M.T.; Mohd Zain, S.N.; Chandrawathani, P.; Premaalatha, B.; Mohd Khairul Nizam, M.K.; Arutchelvan, R.; Suresh, K. Genetic Diversity of Rodent Blastocystis sp. From Peninsular Malaysia. Trop. Biomed. 2018, 35, 586-592.

433. Kalani, H.; Daryani, A.; Fakhar, M.; Sharif, M.; Faridnia, R. A Survey on Intestinal Parasites in Swiss Webster Mice. J. Maz. Univ. Med. Sci. 2013, 22, 63-69.

434. Chamavit, P.; Sahaisook, P.; Niamnuy, N. The Majority of Cockroaches from the Samutprakarn Province of Thailand Are Carriers of Parasitic Organisms. EXCLI J. 2011, 10, 218-222.

435. Dokmaikaw, A.; Suntaravitun, P. Prevalence of Parasitic Contamination of Cockroaches Collected from Fresh Markets in Chachoengsao Province, Thailand. Kobe J. Med. Sci. 2019, 65, E118-E123.

436. Oğuz, B.; Özdal, N.; Orunç Kilinç, Ö.; Değer, M.S. First Investigation on Vectorial Potential of Blattella Germanica in Turkey I Türkiye'de Blattella Germanica'nın Vektörlük Potansiyeli Üzerine Ilk Araştırma. Ank. Univ. Vet. Fak. Derg. 2017, 64, 141-144. [CrossRef]

437. Motevalli-Haghi, S.F.; Shemshadian, A.; Nakhaei, M.; Faridnia, R.; Dehghan, O.; Malekzadeh Shafaroudi, M.; Nejadi Kelarijani, M.; Nikookar, S.H.; Kalani, H.; Fakhar, M. First Report of Lophomonas Spp. in German Cockroaches (Blattella Germanica) Trapped in Hospitals, Northern Iran. J. Parasit. Dis. 2021. [CrossRef]

438. Farah Haziqah, M.T.; Nur Asyiqin, M.N.; Mohd Khalid, M.K.N.; Suresh, K.; Rajamanikam, A.; Chandrawathani, P.; Mohd Zain, S.N. Current Status of Blastocystis in Cockroaches. Trop. Biomed. 2017, 34, 741-745.

439. Natalia, F.; Suwanti, L.T.; Suprihati, E.; Kusnoto; Koesdarto, S.; Srianto, P. Morphological Detection of the Intestinal Parasite Blastocystis sp. in Fresh and Cultured Feces of Pet Sugar Glider (Petaurus breviceps) (Mammalia: Marsupialia: Petauridae) in Surabaya, Indonesia. Philipp. J. Vet. Med. 2018, 55, 91-96.

440. Li, T.-S.; Zou, Y.; Ma, Y.-T.; Ma, Y.-Y.; Chen, H.; Liang, X.-X.; Cong, W.; Sun, X.-L.; Zhu, X.-Q. Molecular Characterization of Eimeria spp. and Blastocystis in Rabbits in Shandong Province, China. Parasitol. Res. 2020, 119, 1547-1551. [CrossRef]

441. Javanmard, E.; Rahimi, H.M.; Niyyati, M.; Aghdaei, H.A.; Sharifdini, M.; Mirjalali, H.; Zali, M.R.; Karanis, P. Molecular Analysis of Blastocystis sp. And Its Subtypes from Treated Wastewater Routinely Used for Irrigation of Vegetable Farmlands in Iran. J. Water Health 2019, 17, 837-844. [CrossRef] 
442. Ithoi, I.; Jali, A.; Mak, J.W.; Wan Sulaiman, W.Y.; Mahmud, R. Occurrence of Blastocystis in Water of Two Rivers from Recreational Areas in Malaysia. J. Parasitol. Res. 2011, 2011, 123926. [CrossRef]

443. Richard, R.L.; Ithoi, I.; Majid, M.A.A.; Wan Sulaiman, W.Y.; Tan, T.C.; Nissapatorn, V.; Lim, Y.A.L. Monitoring of Waterborne Parasites in Two Drinking Water Treatment Plants: A Study in Sarawak, Malaysia. Int. J. Environ. Res. Public Health 2016, 13, 641. [CrossRef]

444. Noradilah, S.A.; Lee, I.L.; Anuar, T.S.; Salleh, F.M.; Manap, S.N.A.A.; Mohtar, N.S.H.M.; Azrul, S.M.; Abdullah, W.O.; Moktar, N Occurrence of Blastocystis sp. in Water Catchments at Malay Villages and Aboriginal Settlement during Wet and Dry Seasons in Peninsular Malaysia. PeerJ 2016, 4, e2541. [CrossRef]

445. Banaticla, J.E.G.; Rivera, W.L. Detection and Subtype Identification of Blastocystis Isolates from Wastewater Samples in the Philippines. J. Water Health 2011, 9, 128-137. [CrossRef] [PubMed]

446. Karaman, Ü.; Kolören, Z.; Seferoğlu, O.; Ayaz, E.; Demirel, E. Presence of Parasites in Environmental Waters in Samsun and Its Districts. Turk. Parazitolojii Derg. 2017, 41, 19-21. [CrossRef]

447. Koloren, Z.; Gulabi, B.B.; Karanis, P. Molecular Identification of Blastocystis sp. Subtypes in Water Samples Collected from Black Sea, Turkey. Acta Trop. 2018, 180, 58-68. [CrossRef]

448. Kolören, Z.; Karaman, Ü. Investigation of Blastocystis Subspecies in Water Samples Collected from Ordu Province. Turk. Parazitolojii Derg. 2019, 43, 111-117. [CrossRef]

449. Isazadeh, M.; Mirzaii-Dizgah, I.; Shaddel, M.; Homayouni, M.M. The Prevalence of Parasitic Contamination of Fresh Vegetables in Tehran, Iran. Turk. Parazitolojii Derg. 2020, 44, 143-148. [CrossRef]

450. Al Nahhas, S.; Aboualchamat, G. Investigation of Parasitic Contamination of Salad Vegetables Sold by Street Vendors in City Markets in Damascus, Syria. Food Waterborne Parasitol. 2020, 21, e00090. [CrossRef]

451. Han, T.-H.; Park, S.-H.; Chung, J.-Y.; Jeong, H.-W.; Jung, J.; Lee, J.-I.; Hwang, Y.-O.; Kim, I.-Y.; Lee, J.-H.; Jung, K. Detection of Pathogenic Viruses in the Ambient Air in Seoul, Korea. Food Environ. Virol. 2018, 10, 327-332. [CrossRef] [PubMed]

452. Jiménez, P.A.; Jaimes, J.E.; Ramírez, J.D. A Summary of Blastocystis Subtypes in North and South America. Parasites Vectors 2019, 12, 376. [CrossRef]

453. Zanetti, A.D.S.; Malheiros, A.F.; de Matos, T.A.; Longhi, F.G.; Moreira, L.M.; Silva, S.L.; Castrillon, S.K.I.; Ferreira, S.M.B.; Ignotti, E.; Espinosa, O.A. Prevalence of Blastocystis sp. Infection in Several Hosts in Brazil: A Systematic Review and Meta-Analysis. Parasit. Vectors 2020, 13, 30. [CrossRef] [PubMed] 